\title{
LASER SPECKLE CONTRAST IMAGING FOR ASSESSING MICROCIRCULATION IN DIABETIC FOOT DISEASE
}

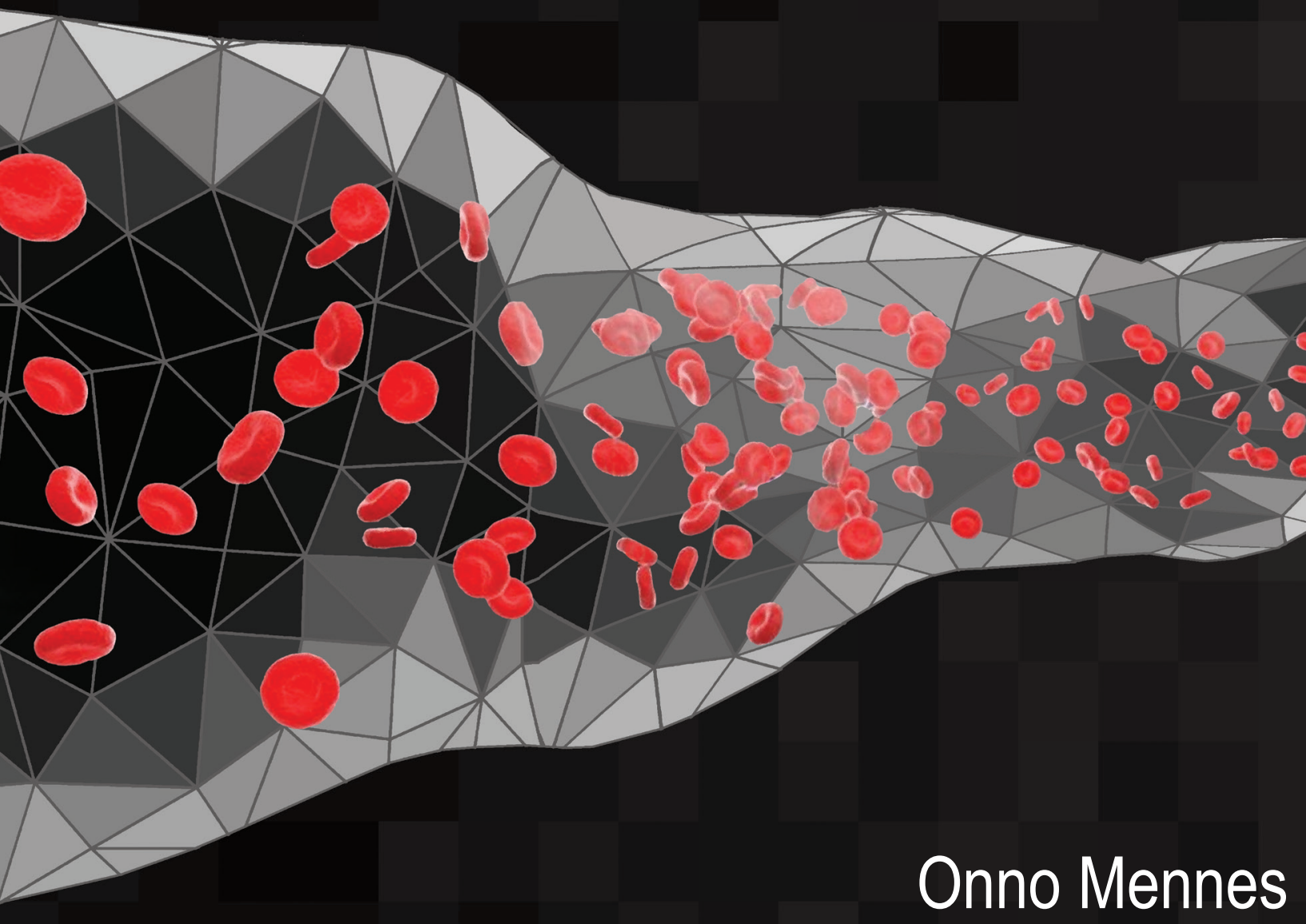





\section{LASER SPECKLE CONTRAST IMAGING FOR ASSESSING MICROCIRCULATION IN DIABETIC FOOT DISEASE}

Onno August Mennes 



\title{
LASER SPECKLE CONTRAST IMAGING FOR ASSESSING MICROCIRCULATION IN DIABETIC FOOT DISEASE
}

\author{
DISSERTATION
}

to obtain

the degree of doctor at the University of Twente,

on the authority of the rector magnificus,

prof. dr. ir. A. Veldkamp,

on account of the decision of the Doctorate Board

to be publicly defended

on Friday 10 September 2021 at 12.45 hours

by

Onno August Mennes

born on the 28th of December, 1989

in Utrecht, The Netherlands 
This dissertation has been approved by:

Supervisors:

prof. dr. R.H.J.A. Slart

prof. dr. ir. W. Steenbergen

Co-supervisor:

dr. J.J. van Netten

Funding: This research was funded by NWO-TTW (project number 14538 ); Pioneers in Healthcare 2015 - A new field for photoacoustic Imaging: From validation in healthy feet to critical limb ischemia detection in people with diabetes; and Diavasc Foundation.

Cover design: O.A. Mennes

Printed by: Gildeprint

ISBN: 978-90-365-5220-2

DOI: https://doi.org/10.3990/1.9789036552202

(C) 2021 Onno August Mennes, Enschede, The Netherlands. All rights reserved. No parts of this thesis may be reproduced, stored in a retrieval system or transmitted in any form or by any means without permission of the author. 


\title{
GRADUATION COMMITTEE:
}

\author{
Chair / secretary: $\quad$ prof. dr. J.L. Herek \\ University of Twente \\ Supervisors: \\ prof. dr. R.H.J.A. Slart \\ University of Twente; \\ University Medical Center Groningen \\ prof. dr. ir. W. Steenbergen \\ University of Twente \\ Co-supervisor: \\ dr. J.J. van Netten \\ University Medical Center Amsterdam; \\ Queensland University of Technology \\ Committee Members: prof. dr. ir. R.M. Verdaasdonk \\ University of Twente \\ prof. dr. C.J.M. Doggen \\ University of Twente \\ prof. dr. M. Nieuwdorp \\ University Medical Center Amsterdam \\ prof. dr. A.W.J.M. Glaudemans \\ University Medical Center Groningen \\ prof. dr. J.G. van Baal \\ Ziekenhuis Groep Twente; \\ University of Cardiff
}





\section{TABLE OF CONTENTS}

$\begin{array}{lll}\text { Chapter } 1 \quad \text { General Introduction } & 9\end{array}$

$\begin{array}{lll}\text { Chapter } 2 & \text { Novel Optical Techniques For Imaging }\end{array}$

Microcirculation In The Diabetic Foot

Chapter $3 \quad$ Assessment Of Microcirculation In The Diabetic Foot

With Laser Speckle Contrast Imaging

Chapter 4

The Association Between Foot And Ulcer

Microcirculation Measured With Laser Speckle

Contrast Imaging And Healing Of Diabetic Foot Ulcers

Chapter 5

Semi-Automatic Tracking Of Laser Speckle Contrast

Images Of Microcirculation In Diabetic Foot Ulcers

Chapter 6

Assessing The Microcirculation Of The Plantar Side Of

The Foot With Laser Speckle Contrast Imaging During

Endovascular Revascularisation Procedures In

Patients With Peripheral Arterial Occlusive Disease

Chapter 7

General Discussion

Chapter 8

Summary

Chapter 9

Samenvatting

Appendices

About The Author

List Of Publications 

GENERAL INTRODUCTION 
Chapter 1 


\subsection{DIABETIC FOOT ULCERS}

Diabetes Mellitus is one of the most common chronic diseases worldwide. According to the International Diabetes Federation, 463 million people worldwide are affected by this disease and $79 \%$ of people with diabetes live in low- and middle-income countries (1). Also, incidence rates of diabetes mellitus are increasing worldwide. Important causes for these rates are a longer life-expectancy and our changing lifestyle (e.g. overweight and obesity, and physical inactivity). These developments are worrisome, because diabetes can be a major cause of serious complications such blindness, heart attacks, strokes, and kidney failures. Another serious and frequent complication of diabetes is diabetic foot disease $(2,3)$.

The International Working Group on the Diabetic Foot (IWGDF) defines a diabetic foot as "Infection, ulceration, or destruction of tissues of the foot of a person with currently or previously diagnosed diabetes mellitus, usually accompanied by neuropathy and/or peripheral arterial disease (PAD) in the lower extremity" (4). Annual incidence rates are estimated around $2 \%$ and life-time incidence is estimated between $19-34 \%$ for people with diabetes (5). Diabetic foot ulcers - defined as a "Foot ulcer in person with currently or previously diagnosed diabetes mellitus and usually accompanied by neuropathy and/or PAD in the lower extremity" (4) - have serious effects on the patient's health. First of all, they are associated with high mortality and morbidity rates. The risk of death at five years for a person with diabetes and a foot ulcer is 2.5 times higher than the risk for a person with diabetes without a foot ulcer. Also, foot ulcers increase the risk of lower limb amputation with approximately $20 \%$, they are often the leading cause of (increased, and long stay) hospitalization. In addition to the health-related consequences, diabetic foot ulcers also lead to substantial costs for the healthcare system. All of these consequences are even more severe in case of diabetic foot ulcers with critical ischemia as a result of reduced tissue perfusion. Therefore, early and accurate diagnosis and treatment of this condition is needed $(5,6)$.

Currently, the diagnosis of (critical-) ischemia consists of visual and manual inspection and non-invasive measurements of blood flow related parameters in the foot, by means of assessing the ankle and toe pressure, or the transcutaneous oxygen pressure $\left(\mathrm{TcpO}_{2}\right)$. The International Working Group on the Diabetic Foot (IWGDF) provided the necessary cut-off values for these measurements to diagnose (critical-) ischemia $(7,8)$. Based on the non-invasive measurements, accurate treatment of diabetic foot ulcers is possible. This treatment relies on five major factors, including relief of pressure and protection and care of the ulcer, restoration of skin perfusion, treatment of infection, metabolic control and treatment of co-morbidities (9). To determine the need of restoration of skin perfusion, one has to determine the patient's vascular status. Based on this vascular assessment, treatment decisions such as the need for 
revascularization can be made (9). However, these current non-invasive assessments have several disadvantages. First, they fail to adequately measure the microcirculation at the site of the ulcer, since the ankle and toe pressure only assess microcirculation in the lower extremities at those specific locations, and while $\mathrm{TcpO}_{2}$ can assess microcirculation locally, this is not necessarily in or closely adjacent to the ulcer. Furthermore, the non-invasive blood pressure measurements can be influenced by other factors such as oedema or infection $(7,10)$. Also circular calcification of the media can stiffen the arterial wall, a common finding in diabetes. This may result in higher measured blood pressure levels and are therefore less reliable in people with diabetes (11). Consequently, an improvement of the assessment of microcirculation in people with diabetic foot ulcers is strongly needed.

\subsection{NOVEL OPTICAL IMAGING TECHNIQUES}

Novel optical imaging techniques (e.g., Laser Speckle Contrast Imaging, Laser Doppler Perfusion Imaging, Photoacoustic Imaging or Hyperspectral Imaging) may be promising to address previously described shortcomings of the currently used measurements. These novel optical techniques can assess the situation at the ulcer location and the microcirculatory changes to the local perfusion. In the ideal case, optical techniques are non-invasive, non-contact, non-ionizing, do not need a contrast agent and they should be both stable and reproducible. Also, these techniques should be suitable for fast imaging of the complete surface of the foot. These aspects are important for a successful implementation in the clinical setting, because it is of utmost importance that the burden on a patient does not increase with the use of advanced imaging techniques. Furthermore, these technologies should be easily integrated in regular outpatient work-up and treatment monitoring.

Despite the promising characteristics of these novel optical imaging techniques for the assessment of microcirculation, none of them have been applied as clinical routine in diabetes foot care. Therefore, more research into these techniques is needed.

\subsection{THE AIM AND OUTLINE OF THIS THESIS}

The overall aim of this thesis is to investigate the potential of novel optical imaging techniques, and in particular the potential of Laser Speckle Contrast Imaging (LSCI), for the assessment of microcirculation in diabetic foot disease and its applicability in the clinical setting. The following research questions will be addressed in this thesis: 
1 What are the advantages and disadvantages of novel optical imaging techniques for local assessment of microcirculation in diabetic foot ulcers?

2 What is the stability and reproducibility of $\mathrm{LSCl}$ for the assessment of microcirculation in diabetic foot ulcers and what is its relation to currently used non-invasive blood pressure measurement?

3 What is the prognostic value of LSCl for healing of diabetic foot ulcers?

4 Can clinical applicability of LSCI be improved with a robust tracking algorithm for semi-automatic analysis of LSCI data?

5 Is LSCl clinically applicable during revascularization procedures and is there a correlation between LSCI outcomes to clinical parameters for the success of revascularization?

Chapter 2 provides a review of several novel optical techniques for imaging microcirculation in the diabetic foot to address the first research question. The following techniques and their applicability for the diagnostic assessment of microcirculation in diabetic foot ulcers will be discussed: Laser Doppler Perfusion Imaging, Laser Speckle Contrast Imaging (LSCl), Photoacoustic Imaging, and Hyperspectral Imaging. Chapters 3 to 6 will particularly focus on LSCI, to address research questions 2 to 5 , respectively. Chapter 3 describes a prospective, single centre, observational cohort study, investigating the stability and reproducibility of $\mathrm{LSCl}$ for the assessment of microcirculation in the diabetic foot. In addition, the relation of $\mathrm{LSCl}$ to the currently used non-invasive blood pressure measurements is examined. Chapter 4 continues with a prospective single centre cohort study. This chapter investigates the prognostic values of $\mathrm{LSCl}$ compared to non-invasive blood pressure measurements in relation to healing of diabetic foot ulcers. In chapter 5 , the development and validation of a fast and robust tracking algorithm for semi-automatic analysis of LSCl data is described. The goal of this tracking algorithm is to facilitate implementation of $\mathrm{LSCl}$ in daily clinical practice. Chapter 6 will focus on the results of a prospective, exploratory, single centre cohort study in which we determined inter-observer variation of LSCl-scans and examined the clinical applicability of $\mathrm{LSCl}$ scans during revascularization procedures. Finally, chapter 7 will discuss the obtained results taking in account the previously formulated research questions, and the limitations of research presented in this thesis. Furthermore, conclusions will be drawn and recommendations for future research and implications for the clinical setting will be given. 


\section{REFERENCES}

1. IDF Diabetes Atlas 9th edition 2019. https://www.diabetesatlas.org/en/

2. Lazzarini PA, Pacella RE, Armstrong DG, van Netten JJ. Diabetes-related lowerextremity complications are a leading cause of the global burden of disability. Diabet Med. 2018;

3. Fowler MJ. Microvascular and macrovascular complications of diabetes. Clin Diabetes. 2011;

4. van Netten JJ, Bus SA, Apelqvist J, Lipsky BA, Hinchliffe RJ, Game F, et al. Definitions and criteria for diabetic foot disease on behalf of the International Working Group on the Diabetic Foot. 2020;19.

5. Armstrong DG, Boulton AJM, Bus SA. Diabetic Foot Ulcers and Their Recurrence. N Engl J Med. 2017;376(24):2367-75.

6. Kerr M, Rayman G, Jeffcoate WJ. Cost of diabetic foot disease to the National Health Service in England. Diabet Med. 2014 Dec 1;31(12):1498-504.

7. Hinchliffe R, Brownrigg J, Apelqvist J, Boyko EJ, Fitridge R, Mills J, et al. IWGDF guidance on the diagnosis, prognosis and management of peripheral artery disease in patients with foot ulcers in diabetes. Diabetes Metab Res Rev. 2016;32:37-44.

8. Hinchliffe RJ, Forsythe RO, Apelqvist J, Boyko EJ, Fitridge R, Hong JP, et al. Guidelines on diagnosis, prognosis, and management of peripheral artery disease in patients with foot ulcers and diabetes (IWGDF 2019 update). Diabetes Metab Res Rev . 2020 Mar 20;36(S1).

9. Bakker K, Apelqvist J, Lipsky B, Van Netten J, International Working Group on the Diabetic foot. The 2015 IWGDF Guidance documents on prevention and management of foot problems in diabetes: development of an evidence-based global consensus. Diabetes Metab Res Rev. 2016;32:2-6.

10. Lipsky BA, Berendt AR, Cornia PB, Pile JC, Peters EJ AD. Infectious Diseases Society of America clinical practice guideline for the diagnosis and treatment of diabetic foot infections. Clin Infect Dis. 2012;54:e132-73.

11. Aerden D, Massaad D, Von Kemp K, Van Tussenbroek F, Debing E, Keymeulen B, et al. The ankle-brachial index and the diabetic foot: A troublesome marriage. Ann Vasc Surg. 2011; 


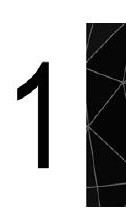





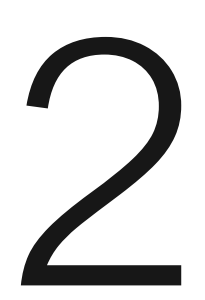

Novel Optical Techniques for Imaging Microcirculation in the Diabetic Foot

\author{
O.A. Mennes, J.J. van Netten, R.H.J.A Slart, W. Steenbergen. \\ Current Pharmaceutical Design 2018; 24, 1-13 \\ (DOI: 10.2174/1381612824666180302141902)
}




\section{ABSTRACT}

Background: The most severe diabetic foot ulcers are those related with critical ischemia, which is primarily diagnosed with non-invasive diagnostics. However, these diagnostics have several disadvantages. For example, they only provide global indications of the (macro)level of ischemia. A potential solution can be found in novel optical imaging techniques for local assessment of the microcirculation in diabetic foot ulcers. This review provides an overview of these imaging techniques (Laser Doppler Perfusion Imaging, Laser Speckle Contrast Imaging; Photoacoustic Imaging and Hyperspectral Imaging) and their applicability for the diagnostic assessment of microcirculation in diabetic foot ulcers.

Method: For each technique the following parts are described: a) their technical background; b) general clinical applications; and, c) its application for microcirculation assessment in diabetic foot ulcers. Parts a-b are based on a narrative review of the literature, part c on a systematic review that was performed in the database Scopus, covering the period from January 1, 2000 to November 31, 2017.

Results: Each of these techniques has specific advantages and disadvantages for imaging microcirculation. Potential clinical use depends on measurement aims, and clinical relevance. However, none of the techniques has a strongly established clinical relevance yet: we found a limited number of publications describing clinical outcomes. Future research is needed to determine which technique is the most clinically relevant for the assessment of microcirculation in diabetic foot ulcers.

Conclusion: Although promising, the currently available novel optical techniques need to be further improved technically and prospective trials are necessary to evaluate their clinical value. 


\subsection{INTRODUCTION}

Diabetic foot ulcers are a major complication of diabetes mellitus, with high morbidity, mortality, and costs (1-4). Yearly incidence is estimated to be around $2 \%$, and life-time incidence lies between $19-34 \%(5-7)$. It is estimated that $50-75 \%$ of all lower extremity amputations are due to diabetes (8). Diabetic foot ulcers are the premium cause of hospitalization in patients with diabetes, and are associated with an increase in hospital admission as well as length of stay $(2,9)$. With a growing number of people with diabetes around the world (10), the burden of complications such as diabetic foot ulcers will continue to grow.

The most severe diabetic foot ulcers are those with critical ischemia: both healing times and length of hospital stay are longest for patients with these ulcers $(1,4)$. The diagnosis of critical ischemia is primarily determined in clinical practice, using noninvasive assessment of blood flow in the feet, by means of assessment of the ankle pressure, toe pressure or transcutaneous oxygen pressure (tcpO2) (6). As a basis for diagnosis, cut-off values for these assessments to diagnose (critical) ischemia are provided in the international guidelines $(6,11)$. These values also provide some information regarding the probability of healing of the ulcer without vascular intervention $(6,12)$. However, current non-invasive measurement systems have various disadvantages.

First, these measurements are not necessarily reflecting the situation of ischemia at the site of the ulcer. With different angiosomes providing blood supply to the feet, low blood flow values in one angiosome may not reflect blood supply at the ulcer location, if that ulcer is found at a different supply region (13). Second, international guidelines indicate that there is a grey area where healing probabilities are unclear (6). For example, toe pressure values between 30 and $50 \mathrm{mmHg}$ indicate a $20-80 \%$ chance of healing without vascular intervention $(11,12,14)$. This uncertainty may result in delayed vascular intervention and increased healing times. A potential explanation for this uncertainty can be found in arteriovenous shunting, frequently seen in patients with diabetes (15). In those patients, microcirculation is affected, but this is not reflected in the ankle pressures or toe pressures used for assessment. Overcoming these shortcomings of some of the non-invasive diagnostics is one of the major challenges in the field of diabetic foot ulcers (6).

A potential solution can be found in novel optical imaging techniques for local assessment of the microcirculation. With such techniques, the situation at the location of the ulcer can be assessed, as well as microcirculatory changes to the local perfusion. Ideally, those measurements should be non-invasive, non-contact, non-ionizing, and they should be able to perform local imaging of the complete surface of the foot in a 
short time procedure $(16,17)$. This is important in the clinical setting, as diagnostic assessment that does not increase the burden on a patient is favoured over advanced imaging techniques such as computed tomography angiography that may pose a significant burden (e.g. radiation dose, iodine-contrast) on a patient, and include longer waiting times as well (6). Furthermore, when quick and adequate diagnostics can be performed during regular outpatient treatment, they can be used directly in treatment plans.

While various reviews (16-20) have been published in recent years focusing on optical techniques to assess microcirculation, these reviews only focused minimally on microcirculation for diabetic foot ulcers (16-20). Microcirculatory assessment for diabetic foot ulcers, however, poses unique challenges, different from other vascular disease areas; these include the combination of the presence of an open wound, local microvascular changes (including arteriovenous shunting), potential presence of macrovascular disease, calcifications, infection, oedema, and poor collateralization (15).

The aim of this review is to provide an overview of novel optical imaging techniques that could possibly be used as a replacement of or to complement the currently used blood pressure measurements for the diagnostic assessment of microcirculation in diabetic foot ulcers.

In this paper, the following optical techniques will be discussed: (1) Laser speckle based perfusion imaging (1a: Laser Doppler Perfusion Imaging (LDPI), and 1b: Laser Speckle Contrast Imaging ( $\mathrm{LSCl})$ ), (2) Photoacoustic Imaging (PAI), and (3) Hyperspectral Imaging ( $\mathrm{HSI}$ ). For each technique, we will discuss its technical background, general clinical application, and specific applications (or potential) for the assessment of microcirculation in diabetic foot ulcers.

\subsection{METHODS}

Based on existing literature (16-20), and our research and clinical experience, four novel optical imaging techniques were selected as topic in this review, as these can be used to measure the microcirculation in tissue: LDPI, LSCI, PAI, and HSI. For each technique a global technical background will be given (part a). Furthermore, one or more general clinical applications of each technique will be described, to present the usefulness in different clinical fields and their possibilities (part b). Finally, to specifically investigate each novel optical technique for imaging microcirculation in diabetic foot ulcers (part c), a systematic review of the literature was performed using the database Scopus (www.scopus.com), covering the period from January 1, 2000, to November 31,2017 . Scopus was chosen because of its multidisciplinary scope. The technological 
background of the different optical techniques and the clinical relevance in a clinical setting or study led to the need of a database that covered both articles in the medical field and articles outside medicine (e.g. engineering). We did not include publications before 2000, as both the technological development and the clinical relevance are rapidly changing.

This study's search strategy was based on the six-stage methodological framework of Arksey and O'Malley (21). One investigator (OAM) searched and selected relevant studies. The main components of the conducted search were (1) microcirculation, (2) diabetic foot ulcer and (3) the optical imaging techniques selected for this review (LDPI, $\mathrm{LSCl}, \mathrm{HSI}$, or PAI). Relevant keywords and synonyms were used to combine the three components. The full search strategies are given in Appendix I. Other inclusion criteria were that the articles had to be written in English and published in peer-reviewed journals. In addition to the database search, reference lists of included publications were manually searched for relevant papers based on title and abstract. All relevant studies were included for a full text review by the investigators to determine inclusion or exclusion in this paper. Results from included studies were summarized by one investigator (OAM), and independently checked by another investigator (JJvN).

\subsubsection{Laser speckle based perfusion imaging}

\subsubsection{Technical background}

Laser speckle based perfusion imaging techniques (e.g. LDPI and LSCI) use a speckle pattern to measure movement or flow in tissue. When coherent laser light illuminates tissue, the resulting reflection creates a detectable destructive and constructive interference pattern called speckle pattern. Light from different parts of the surface of the tissue reflects in different ways to the detector. The measured intensity of the reflected laser light for each point is based on the addition of all wave amplitudes of the light reaching this point. If the individual waves interfere destructively, the resultant intensity is zero and this will be seen as a dark spot in the speckle pattern. In all other locations of the speckle pattern the waves interfere more or less constructively, leading to a nonzero intensity: these are the bright speckles. Furthermore, the reflecting laser light will be changed by the Doppler effect if the light has interacted with moving structures inside the medium $(22,23)$. Relative movement between an object that reflects the laser light (e.g. red blood cells) and the detector, generates a change in frequency of the light. For each individual interaction this frequency change, or Doppler shift, depends on the direction and speed of motion. In practice, light can undergo multiple Doppler shifts, also depending on the blood content of the tissue $(23,24)$. The final result is a dynamic speckle pattern.

LDPI and LSCI both use the dynamic speckle pattern to measure blood flow or microcirculation in tissue. The two techniques, however, differ how they acquire and process data. LDPI uses a fast detector or high-speed camera to capture the dynamic 
speckle pattern in order to measure all the fluctuations in intensity of the reflected laser light. The average frequency of these fluctuations provides information about the average speed of red blood cells, while the magnitude of intensity fluctuations is used to estimate the blood concentration. The tissue perfusion is then estimated as the product of estimated average speed and estimated blood concentration. The penetration depth of LDPI depends on the laser wavelength. Typically, wavelengths of red light $(633 \mathrm{~nm})$ or near infrared light $(780 \mathrm{~nm})$ are used and a penetration depth of 1-2 $\mathrm{mm}$ is achieved. This depth is sufficient and can be used to assess the microcirculation in the capillaries and underlying arterioles and venules and arteriovenous anastomoses deeper in the dermis of the skin (Figure 1) $(16,24,25)$. However, thickened layers of callus or necrotic tissue can affect the measurement and need to be debrided first, and when debridement cannot take place, measurements may not be reliable at the location of the affected skin.
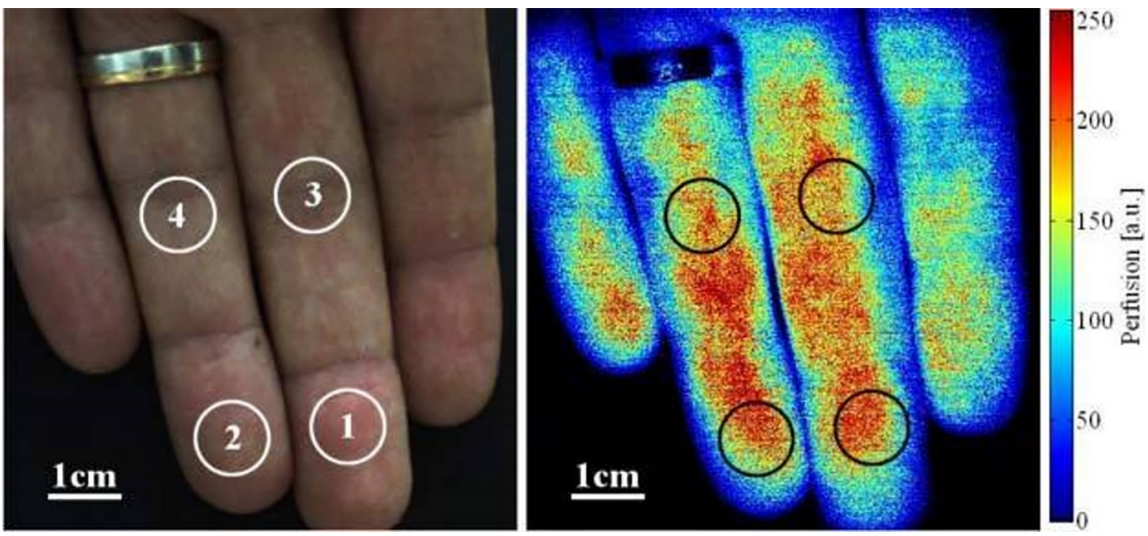

Figure 1 "Color image of fingertips and color-coded blood perfusion map." by Leutenegger et al. (26) licensed under CC BY 3.0

In contrast to LDPI, LSCI uses a camera with a slow response time to capture the dynamic speckle pattern. Consequently, individual speckles will be less clearly visualized, and the contrast of the speckle pattern image is reduced: the contrast in areas with more movement is lower compared to areas with less movement $(24,25,27)$. Draijer et al. (27) and Briers et al. (25) showed that, based on the quantity of measured contrast in an area, an indication can be obtained of the severity of movement present during the measurement. For medical purposes this is crucial: movement measured in tissue is related to blood flow of the moving red blood cells, and provides opportunities to use LSCl as a tool to measure tissue perfusion (Figure 2) (28). While LDPI is regarded as giving more quantitative perfusion estimations than LSCI, LDPI for real time imaging is also more expensive since a high-speed camera is needed. 

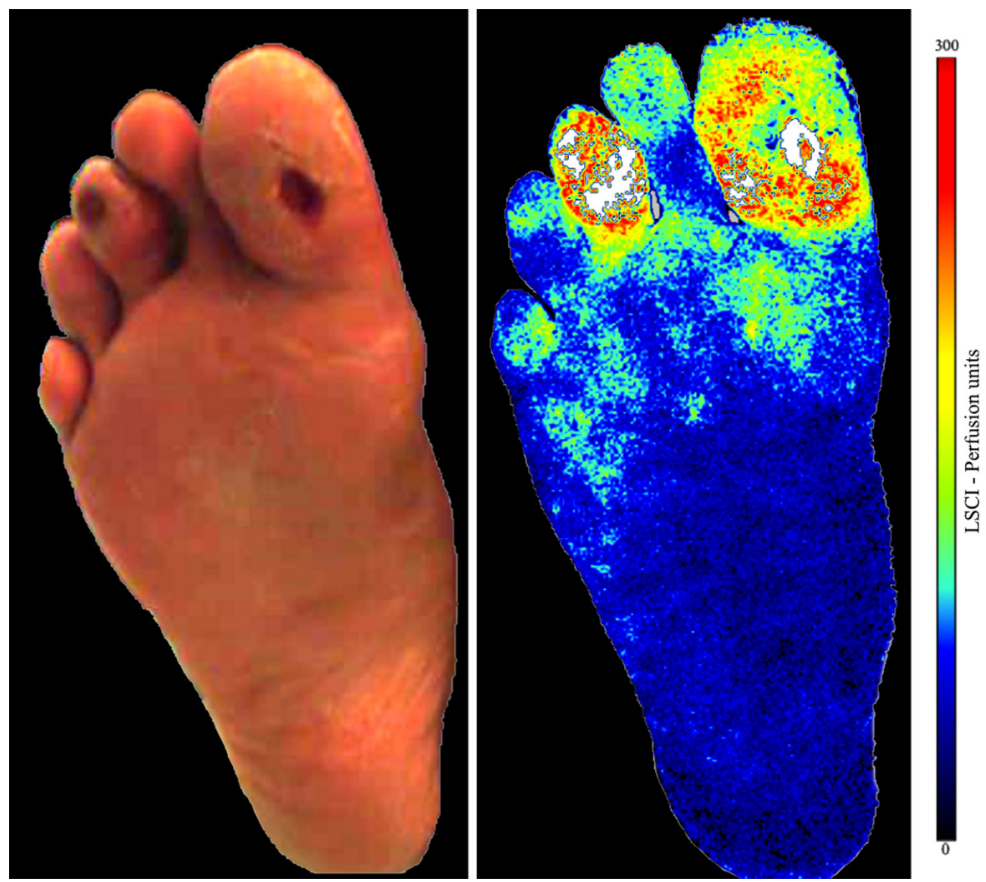

Figure $2 \mathrm{LSCl}$ color image of diabetic foot with ulcer on dig 1 and 3 and color-coded blood perfusion map with increased perfusion values in ulcer area (Pericam PSI). Source: Mennes, O.A., 2016. Color image of diabetic foot and LSCI perfusion map. [photograph] (personal collection).

\subsubsection{Clinical application of LDPI}

LDPI has several clinical applications. Primarily it is used for the assessment of burn depth, but it is also applied in other medical fields such as surgery, wound healing, and for general vascular diagnostics (16).

Regarding the assessment of skin burn depth, Hoeksema et al. (29) conducted a study in which they included 40 patients with intermediate depth skin burns to compare and evaluate the LDPI measurements with clinical assessment on days $0,1,3,5$ and 8 . They found an accuracy of skin burn depth assessments using LDPI of $54 \%$ to $100 \%$ while clinical assessment accuracies were lower, namely $41 \%$ to $100 \%$. Furthermore, they found that the LDPI accuracy was significantly higher than clinical accuracy on day $3(p<0.001)$ and day $5(p=0.005)$. This indicated that burn depth assessment with LDPI was more reliable than clinical evaluation and, therefore, the authors proposed that all burns of intermediate depth should be analysed with a combination of both LDPI scanning and clinical evaluation. These findings were confirmed by Pape et al. (30) and Monstrey et al. (31). They conducted a combined large study among 137 patients with skin burns. In total, they measured 433 different burn sides. Based on their measurements, they created a validated coding technique to predict the 
healing potential (in days) for individual patients. According to the authors, LDPI can be used as a valid tool for the clinical assessment of skin burns.

Anderson et al. (32) used LDPI to quantify blood flow responses to iontophoresis of vasoactive agents; these LDPI findings were then used to examine whether microvascular vasodilation is impaired in patients with systemic sclerosis compared with patients with primary Raynaud's phenomenon and a healthy control group. They concluded that LDPI was feasible to use in measuring blood flow responses to iontophoresis in patients with systemic sclerosis or Raynaud's phenomenon, and that in patients with established systemic sclerosis both endothelium-dependent and endothelium-independent vasodilation are impaired. Furthermore, they recommended using LDPI in order to examine pathophysiology in other groups of patients with rheumatic diseases affecting the microvasculature, and in patients with different severities and durations of scleroderma-spectrum disorders including patients with undifferentiated connective tissue disease. They also indicated that studies to investigate the reproducibility of this technique are still needed (32). In case reproducibility is demonstrated, LDPI could be used (together with symptom-based measurements) to study disease progression over time and responsiveness to vasoactive treatment. Hughes et al. (33) also used LDPI to measure microcirculation in 16 patients with systemic sclerosis. They examined the blood flow response to topical glyceryl trinitrate compared to placebo in fingertip digital ulcers, looking for differences in pathophysiology between fingertip and extensor lesions.

Another example for the clinical use of LDPI is in the prediction of wound healing after surgical interventions. For instance, Ljung et al. (34) used LDPI to measure microcirculation in the skin around the elbow before and after a total elbow replacement in five rheumatoid arthritis patients to ascertain the possible influence of a compromised blood flow on the wound healing process. Eichhorn et al. (35) also used LDPI scans in wound healing, but then in the field of plastic and reconstructive surgery. They performed measurements and simultaneous two-dimensional imaging of the microcirculation $24,48,72 \mathrm{~h}$ and 5 and 14 days postoperatively in 20 patients, who had had reconstruction procedures performed using random or axial pattern flaps. Eichhorn et al. (35) concluded that the two-dimensional LDPI was a useful technique for follow-up and planning of flaps in plastic and reconstructive surgery. They valued the fact that this technique was a simple and non-invasive method and that it included the possibility of obtaining a representative, real-time image of flap microcirculation for comparison with the surrounding area.

\subsubsection{LDPI and the diabetic foot}

The structured search with the combined search terms of "laser Doppler", "diabetic foot" and "(micro)circulation" resulted in 115 hits between 2000 and 2017. Six studies could be included in this review because the majority of the reported studies did not 
use LDPI, but single point laser Doppler flowmetry, or they were not focused on microcirculation. A description of the six included studies follows.

Although LPDI is not commonly used to measure and assess the levels of ischemia in diabetic foot ulcers, multiple studies are done where LDPI is used to measure the effect of different healing techniques for the diabetic foot ulcer. For example Newton et al. (36) included five patients (seven ulcers) with diabetes type 2 and used LDPI to measure microvascular blood flow before and after two, five, and eight weeks of diabetic foot ulcer treatment with dermal replacement. They found an increase in blood flow of $72 \%$ in the base of five out of the seven ulcers studied. They stated that the found changes in blood flow observed in this study might reflected the different angiogenesis in the granulation tissue or vasodilatation of already existing vessels and that LDPI made it possible to detect and assess those changes. Mirimoto et al. (37) found similar results when they measured the vascularization in dermal substitutes for diabetic foot ulcers. They measured the microcirculation of 11 diabetic foot ulcers with LDPI (Moor LDI2-IR; Moor Instruments Ltd., Axminster, Devon, UK, Figure 3) before and after the placement of a dermal substitute. In this way they were able to measure and compare the different ulcers and assess the microcirculation of the ulcer and its surrounding tissue. Although they did not use the LDPI to predict healing tendency or to diagnose the diabetic foot ulcer, they showed that LDPI was able to measure the circulation in diabetic foot ulcers and that this technique could be used for the assessment of microcirculation (37).
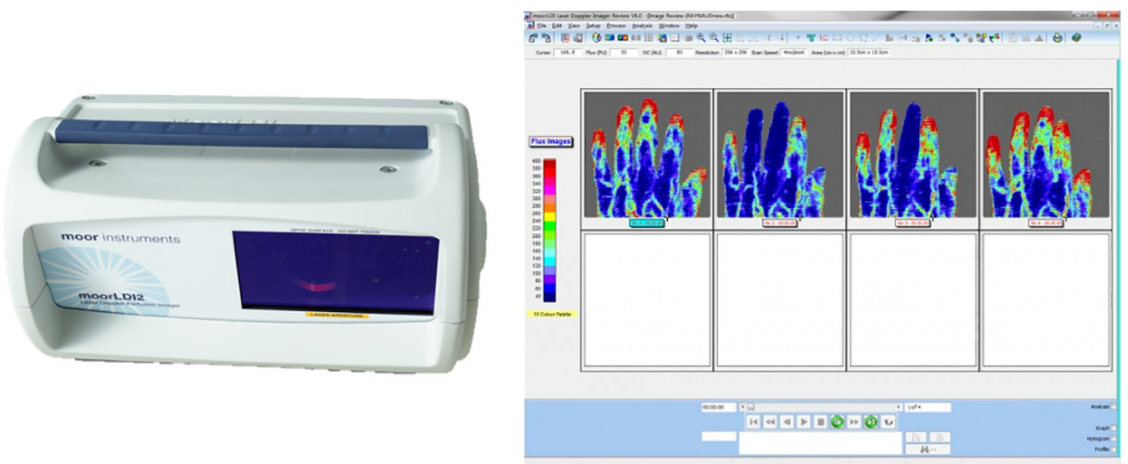

Figure 3: LDPI device (Moor LDI2-IR; Moor Instruments Ltd., Axminster, Devon, UK), as used in the study of Mirimoto et al. (37). Source https://us.moor.co.uk/

Another study that highlighted the usefulness of LDPI for the diabetic foot population was conducted by Caselli et al. (38). They used LDPI to measure the vasodilatory response of methyl nicotinate and compared it to the vasodilatory response to acetylcholine and sodium nitroprusside in ten healthy subjects and ten diabetic patients. In this study they used LDPI to measure the vasodilation and microcirculation 
in the forearm and dorsum of the foot. Although their primary research aim was to measure the vasodilation response of different drugs, the usefulness of LDPI to measure microcirculation in the diabetic foot was shown. For instance, they were able to measure multiple perfusion values of the foot (e.g. base perfusion, peak response to drug, increase in perfusion, area under the curve and time to peak), and found a significant difference between the diabetic population and the healthy control group. This could be an indication that LDPI might be useful for measuring perfusion in a diabetic foot population.

Golster et al. (39) conducted a study to identify whether type 1 diabetes patients (children and adolescents) had signs of microvascular dysfunction and to find a correlation between their microcirculation and different disease parameters (e.g. metabolic control and peripheral nerve dysfunction). They measured the skin blood flow on the dorsum of the foot with LDPI in 37 patients and ten control subjects without diabetes. They performed standard baseline perfusion measurements and applied a stress test to measure post occlusive hyperaemia. They found a correlation between HbA1c (glycated haemoglobin) and blood flow in the foot. Patients with poor metabolic control (acute $\mathrm{HbA} 1 \mathrm{c}>7.5 \%$ ) had an increase in skin blood flow at rest, and when the leg was lowered below heart level, the change in blood flow was significantly reduced. This suggests that low resting blood flow levels contribute to the absence of postural vasoconstrictor response. Golster et al. (39) concluded that LDPI can be used to detect subclinical abnormalities of the microcirculation in young type 1 diabetes.

The final two studies included study population consisting of diverse populations. As their results can be insightful for diabetic foot diagnostics, we did include them in this review. One study was conducted by Gschwandtner et al. (40). They used LDPI to measure the circulation in ischemic ulcers. Particularly, they investigated the relation between the LDPI signal and a visual inspection with capillary microscopy. Only ten out of the 25 included patients were diabetic foot patients. Gschwandtner et al. (40) found that changes in the circulation measured by LDPI may coincide with changes in the number of visible capillaries within an ischemic ulcer. This can be an indication that LDPI is applicable for the assessment of ischemia in (diabetic) ischemic foot ulcers. The second study, conducted by Petrofsky et al. (41), investigated the effect of electrical stimulation on perfusion in the skin of the hand, leg and foot of patients with wounds (half of the patients had type 2 diabetes). They found an increase in blood flow in the skin after an electrical stimulation. An increase of $53 \%$ was found in the blood flow during the electrical stimulations and this was detectable with LDPI.

\subsubsection{Clinical application of $L S C I$}

LSCI has mainly been applied in clinical pilot studies or pre-clinical studies (such as animal testing) to measure the cutaneous, cerebral and renal blood flow (16). For example, Mirdell et al. (42) measured perfusion with $\mathrm{LSCl}$ in 34 patients with burns at 
regular intervals after injury until complete reepithelialisation or surgery, and found a significant difference in perfusion between burns that healed early ( $<7$ days) and burns that healed after $>12$ days or for which surgical intervention was needed. Van Vuuren et al. (43) used LSCI for the non-invasive assessment of blood flow of cutaneous wounds. They examined if $\mathrm{LSCl}$ could be used as a predictor of venous ulcer healing. A sensitivity of $92.3 \%$, specificity of $75.0 \%$, positive predictive value of $80.0 \%$, and negative predictive value of $75.0 \%$ was found in predicting wound healing based on laser speckle images. The wound edge proved to be the best predictor for wound healing. Furthermore, Hecht et al. (44) used non-invasive intraoperative LSCI to predict infarction in 22 patients while they underwent surgery for malignant hemispheric stroke. Bezemer et al. (45) evaluated the use of LSCI for mapping renal cortical microvascular perfusion. They found that $\mathrm{LSCl}$ was useful to detect differences in reperfusion dynamics following different durations of ischemia and that renal microvascular perfusion heterogeneities could be assessed by LSCI (45). In another study, LSCI was used to visualize the microcirculation for real time cerebral blood flow measurement (46) or to measure the optic nerve head blood flow to diagnose for example preperimetric glaucoma (47).

Some review papers evaluated the usefulness of $\mathrm{LSCl}$ in a clinical setting, for example Humeau-Heurtier (48) and Roustit et al. (49). Roustit et al. (49) reviewed the advantages and limitations of the main skin provocation tests, combined with measurement techniques such as LSCI. They concluded that LSCI, when coupled with reactivity tests (e.g. post-occlusive reactive hyperaemia, local thermal hyperaemia, pressure-induced vasodilation, and iontophoresis of vasodilators), offered easy to perform and reliable tools to assess endothelial and neurovascular function in the cutaneous circulation (49). Humeau-Heurtier et al. (48) described the relevance of LSCI for assessing the vascular function in patients. Besides the usefulness of different evaluation techniques feasible with LSCI (e.g. Postocclusive hyperaemia, local thermal hyperaemia or microdialysis), they described multiple clinical opportunities (for example; measurements of sleep apnoea, exercise training or antiangiogenic therapy) for laser techniques of clinical and treatment efficacy (48).

Finally, Zhang et al. (50) integrated hyperspectral, LSCI, and thermographic imaging modalities in a single-experimental setup for non-invasive monitoring of tissue oxygenation, perfusion, and thermal emission during the wound healing process. In their experiment, they monitored the healing process of a wound with $3 \mathrm{~mm}$ diameter that was induced on a healthy subject's lower extremity. They were able to identify the different phases of the wound healing process and provided quantitative guidance for different therapeutic options that could be used for diabetic foot ulcers. 


\subsubsection{LSCl and the diabetic foot}

The structured search with the combined search terms of "laser speckle", "diabetic foot" and "circulation" resulted in a total of 69 documents between 2000 and 2017. Even though some studies were performed to measure circulation in the foot, no studies were found that focused specifically on the measurement of the microcirculation of diabetic foot ulcers with LSCI. This shows that, despite the potential usefulness as discussed in the previous paragraph, LSCI has not yet been studied in people with diabetic foot ulcers. This is an important area for future research.

However, we found two studies closely related to diabetic foot ulcers. Hellmann et al. (51) recruited 12 healthy subjects and 12 persons with type 2 diabetes and used LSCI (PeriCam PSI System, Perimed, Järfälla, Sweden. Figure 4) to determine whether treprostinil iontophoresis increased skin microcirculation. They excluded patients with an active diabetic foot ulcer. They used LSCI to measure the microcirculation in people and showed that it could be used to diagnose small changes in microcirculation in specific areas, such as the skin on the foot or ankle. Based on these results, it can be hypothesized that LSCI may be used similarly in people with diabetic foot ulcers.

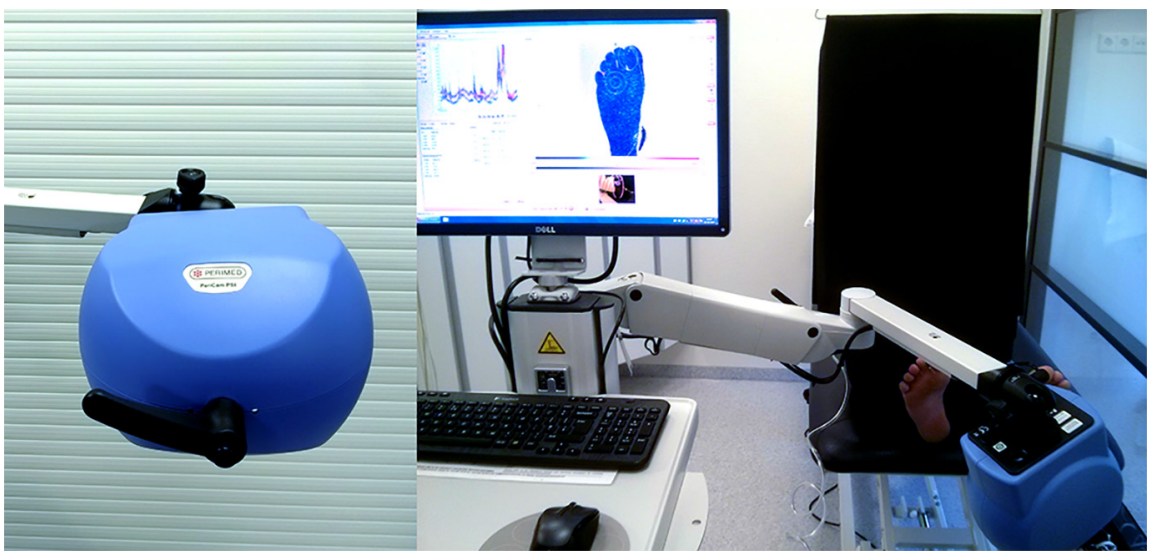

Figure 4: LSCI device (PeriCam PSI System, Perimed, Järfälla, Sweden). Source: Mennes, O.A., 2016. PeriCam PSI System. [photograph] (personal collection).

Furthermore, a more recent study from Katsui et al. (52) showed the potential usefulness of $\mathrm{LSCl}$ in the clinical setting. They used LSCI to assess heating-induced microcirculatory fluctuations in the proximal and distal sites of the dorsal foot of 36 patients with peripheral artery disease (PAD). Of their included PAD patients, $67 \%$ had diabetes mellitus. This means that their results are likely applicable to the diabetic foot population. They found that the LSCI thermal load test discriminated transcutaneous oxygen tension $<30 \mathrm{mmHg}$ with good sensitivity (78.7\%) and specificity (96.2\%). Accordingly, Katsui et al. (52) concluded that LSCI can be used to measure 
microcirculation of the foot and they expect that it can serve as a useful non-invasive method for diagnosing severe limb ischemia. In conclusion, these two studies showed the potential of LSCI for the assessment of microcirculation in the diabetic foot population, however such a study is not yet performed.

\subsubsection{Hyperspectral Imaging}

\subsubsection{Technical background}

Hyperspectral imaging (HSI) is based on optical spectroscopy. HSI uses linearly polarized white light to illuminate the tissue. For the light, three options exist: 1) it can be reflected by the skin, or 2) it penetrates the skin and is absorbed by the tissue, or 3 ) it is scattered and re-emitted by the tissue (53). A part of the reflected light or reemitted light will be detected. Based on the polarization of the light, a discrimination can be made between light reflected by the skin (which is still polarized) or light reemitted from the tissue (which has lost its polarization) (53). By selecting light polarized perpendicular to the polarization of the illuminating light, HSI can select the light that has penetrated into the tissue and exclude reflections from the tissue surface. From the reflected light a spectrum can be acquired and stored in a so-called hypercube. In this way, each pixel in this hypercube represents the local reflectance value at a specific wavelength, reemitted at a specific location on the tissue surface. Based on the measured spectrum and knowledge on light transport in the medium, an absorption spectrum of the tissue can be determined. Because the absorption spectra of many tissue chromophores are known, the characteristics of the tissue can be measured in terms of chromophore content (53-55). Blood volume and oxygen saturation of blood in dermis can be measured as such. For example, deoxyhaemoglobin has a single absorption peak around $554 \mathrm{~nm}$, while oxyhaemoglobin has two absorption peaks, around 542 and $578 \mathrm{~nm}$ (56). Based on these differences, spectroscopic measurements can be performed to visualize microcirculation and local blood oxygen saturation in tissue (Figure 5) (53). 


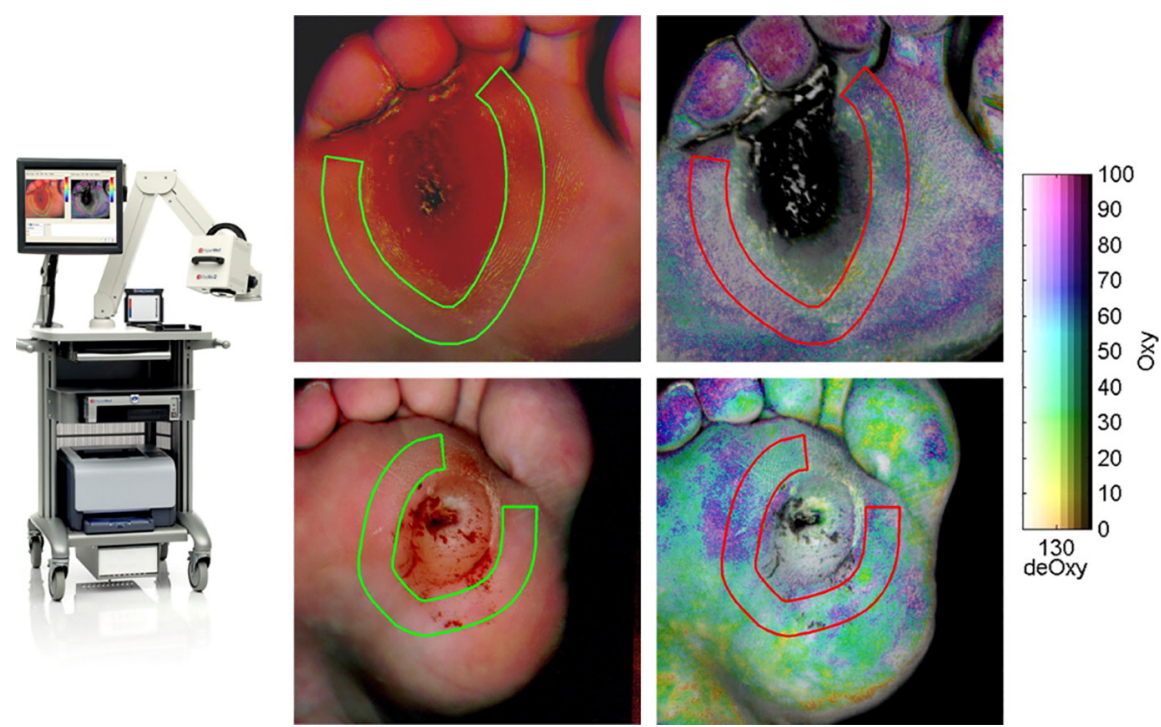

Figure 5: "RGB and hyperspectral imaging (HSI) of a healing diabetic foot ulcer taken with the HTOM system. The top panels show a healed diabetic foot ulcer case. HTOM values are 75,34 , and 69\% for oxy, deoxy, and StO2, respectively. The bottom panels show a non-healed diabetic foot ulcer case. HTOM values are 60, 53, and 53\% for oxy, deoxy, and StO2, respectively. Tissue oxygenation is higher in the healed ulcer as seen by the more purplish tone compared with the more cyan/green tone. Mean oxy and deoxy values were determined for each ulcer from an approximate 1-cm-thick band drawn within the periwound area." by Nouvong A, et al. (57) licensed under CC BY 3.0

\subsubsection{Clinical application of HSI}

HSI bears various possibilities for clinical practice (58). For example, Ferris et al. (59) used HSI to complement in the diagnosis of cervical neoplasia. They included 111 nonpregnant women and compared the results of an abnormal screening Pap smear (or Pap test: screening procedure for cervical cancer (60)) with an HSI scan of the cervix. They found that both techniques had the same specificity (70\%) but that the sensitivity or HSI was higher (97\%) than for the currently used Pap smear screening (72\%). In this way HSI detected cervical cancer precursors at a greater rate than that of the currently used Pap smear screening (59). A more recent study by Wang et al. (61) confirmed those results. Wang et al. also investigated the possibilities of HSI for cervical neoplasia diagnosis in a study among 26 patients with cervical abnormalities (61). They compared the results of $\mathrm{HSI}$ with histologic measurements and found that both methods showed great similarities in their diagnostic power (61). Other examples for the usefulness of $\mathrm{HSI}$ in cancer diagnostics are shown in the field of breast cancer (62), prostate cancer (63) and skin cancer (64), regarding the clinical applicability of HSI. However, these studies were conducted among animals (rats or mice) and results may therefore not necessarily translate to clinical practice. 
Another example of an animal study that indicated some potential usefulness of HSI in a clinical setting was from Gillies et al. (65). They used HSI to monitor changes in skin oxygenation during haemorrhagic shock in pigs. They were able to analyse the changes in skin oxygenation during shock and resuscitation in an objective manner with HSI. Cancio et al. (66) performed a similar study. They included seven pig models to measure the effect of a haemorrhagic shock. They found that HSI was a promising non-invasive and non-contact tool for quantifying changes in skin oxygenation during haemorrhagic shock and resuscitation. However, the results of both studies have not yet been confirmed in humans.

$\mathrm{HSI}$ has been used to measure oxygen saturation values in the temporal retinal arterioles and venules in healthy volunteers. A study among 14 normal volunteers showed that the mean oxygen saturation of the temporal retinal arterioles was 104.3 $( \pm 16.7)$, and in the venules $34.8( \pm 17.8)$. This study provided an overview of the normal values of oxygen saturation in the retia and this might help in the diagnosis of retinal vascular occlusion (67). Additionally, Neville et al. (68) made a database with a normative range of oxyhaemoglobin, deoxyhaemoglobin, and oxygen saturation of 11 anatomical regions. This database was based on the values of 194 healthy subjects. For each of the 11 regions, a HSI oxygenation map was made. Saturation levels were higher at plantar and palmar regions. During occlusion tests, a decrease in perfusion levels was found and this simulated the effect of ischemia. Neville et al. (68) concluded that $\mathrm{HSI}$ was a reliable technique that may help in the diagnosis and treatment of vascular diseases. In line with this study, Chin et al. (69) also investigated if HSI could diagnose the presence or absence of peripheral artery disease (PAD) and if it could predict the severity of the disease. They included 46 healthy (no-PAD) subjects and 65 PAD-patients. Some significant differences in the deoxyhaemoglobin values for the plantar metatarsal, arch, and heel angiosomes were found between patients with and without PAD. Furthermore, a significant correlation between the deoxyhaemoglobin and the ankle-brachial index (commonly used to diagnose PAD) was found. Based on their results they suggested that HSI was able to diagnose the presence of PAD and that it might evaluate the severity of the disease.

\subsubsection{HSI and the diabetic foot}

Our structured search with the combined search terms of "hyperspectral", "diabetic foot" and "(micro)circulation" resulted in 86 hits between 2000 and 2017. Six papers were included in our review, as these were the ones in which HSI was specifically applied to the diabetic foot. The majority of the other papers were focused on other contexts (such as wound healing, burn depth assessment or animal studies) or they were not focused on microcirculation. A description of the included papers follows. 
Greenman et al. (70) included 108 people (87 diabetes mellitus patients and 21 healthy controls) and used $\mathrm{HSI}$ to investigate haemoglobin saturation in the forearm and foot. They indicated that haemoglobin saturation in the foot of people with diabetes was reduced in comparison to the control group. This impairment was further accentuated when there was presence of neuropathy.

In a study by Khaodhiar et al. (71), prediction of diabetic foot ulcer healing was investigated. They included ten persons with type 1 diabetes with 21 foot ulcer sites in total, 13 persons with type 1 diabetes without ulcers, and 14 non-diabetic control subjects. They used medical HSI (CombiVu-R Hyperspectral Technology (HyperMed) to measure the oxyhaemoglobin and deoxyhaemoglobin at or near the ulcer area and on the upper and lower extremity distant from the ulcer. They found that HSI has the capability to identify microvascular abnormalities and tissue oxygenation in the diabetic foot. Another finding was that both oxyhaemoglobin and deoxyhaemoglobin were significantly lower (respectively 38 and 26 vs 50 and 49 arbitrary units) in patients with nonhealing ulcers than in healing ulcers. Furthermore, HSI was able to predict foot ulcer healing with a sensitivity of $93 \%$ and a specificity of $86 \%$.

Nouvong et al. (57) performed a prospective single-arm blinded study with 66 patients with diabetes mellitus. The relative concentration of superficial tissue oxyhaemoglobin and deoxyhaemoglobin was measured with $\mathrm{HSI}$ oxygenation mapping (OxyVu; HyperMed, Burlington, MA, Figure 5) from intact tissue bordering the ulcer. A "healing index", derived from oxyhaemoglobin and deoxyhaemoglobin values, was used to assess the potential for healing. Sensitivity of $80 \%$ and specificity of $74 \%$ was found; those numbers increased to respectively $86 \%$ and $88 \%$ after post-hoc removal of three false-positive and four false-negative cases. However, the exact nature of their "healing index" has never been published, which means the findings cannot be reproduced.

Furthermore, a pilot study ( $\mathrm{n}=2$ ) from the same research group (72) showed the results of a combination of $\mathrm{HSI}$ and an experimentally validated skin optical model to monitor feet of two patients with diabetes. They aimed to observe temporal changes in local epidermal thickness and oxyhaemoglobin concentration, to gain insight into the progression of foot ulcer formation and healing. They observed the ulcers from becoming apparent to the naked eye until they healed and closed. An increase in oxyhaemoglobin concentration around the ulcer was found as well as changes is the epidermal thickness. Both effects might be useful for early prediction of diabetic foot ulceration in a clinical setting.

Finally, Jeffcoate et al. (55) explored the use of HSI to predict healing of diabetic foot ulcers in patients with diabetes. They included 43 patients with a diabetic foot ulcer and measured oxygenation with $\mathrm{HSI}$. Their findings suggest that HSI may predict the healing tendency of the ulcer in routine practice. There was a negative association 
between tissue oxygenation assessed by $\mathrm{HSI}$ at baseline and healing by 12 weeks $(p=0.009)$. The mean arbitrary HSI units at the ulcer site was significantly lower $(p=0.016)$ in the healed ulcer group than in the not healed ulcer group (47.9 vs 61.9 arbitrary units). This is in contrast with the earlier published studies of Khaodhiar et al. (71) and Nouvong et al. (57), who found a positive association between tissue oxygenation and ulcer healing. Jeffcoate et al. explained their negative correlation with the hypothesis that it could be caused by a reduced delivery of oxygen to extravascular tissue or from macrovascular shunting leading to a reduced microvascular transit time that would impair the release of oxygen to the tissues that led to an increase in oxyhaemoglobin at microvascular level. However, evidence for this hypothesis is limited. More research to investigate these interesting contradictory findings is necessary.

\subsubsection{Photoacoustic Imaging}

\subsubsection{Technical background}

Photoacoustic Imaging (PAl), sometimes referred to as optoacoustic imaging, images tissue based on optically induced ultrasound waves. When tissue is illuminated by short laser light pulses (typically 5-100 nanoseconds), the locally absorbed light generates a temperature rise. Since this happens in a short time, a local mechanical stress is generated (the thermo-elastic effect) resulting in the emission of acoustic waves $(73,74)$. Those acoustic waves can be detected as ultrasound and they can be used with a reconstruction algorithm to visualize the locations where light absorption took place (Figure 6). Because the optical absorption coefficients of different tissue types depend on wavelength of the used laser light, it is possible to target specific types of tissue (e.g. oxy- or deoxyhaemoglobin, melanin or water) with properly chosen wavelengths. For example by measuring the ultrasonic response of light pulses at different wavelengths, the location of blood vessels with high oxyhaemoglobin or deoxyhaemoglobin and a discrimination between arteries and veins can be made $(73,74)$. Furthermore by using exogenous contrasts, molecular PAl enables visualizing specific cellular functions and molecular processes, to further enhance the imaging signal, especially those materials with strong absorption in the near-infrared (NIR) region where optical attenuation is weaker than the visible light region in vivo. In recent years, great efforts have been devoted to enhance the molecular imaging capability of PAI, and considerable progress has been made in optimizing both the PAl systems for better detection sensitivity, and the contrast agents for better contrast enhancement (75-78). A limitation of PAI may be that the probe should be in physical contact with the tissue, the measurements cannot be done in a non-contact way. 

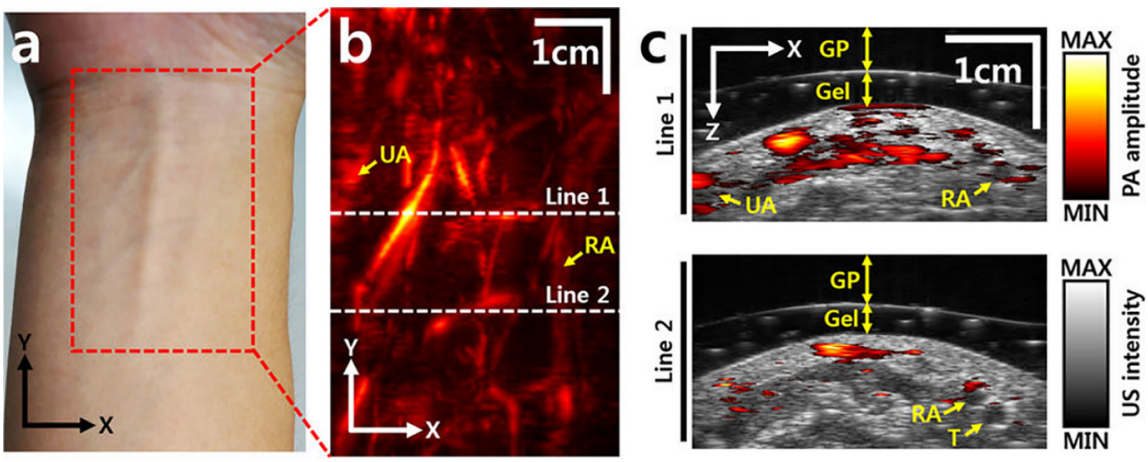

Figure 6A: Photograph and B: PAI MAP image of a human right forearm. The red dashed rectangle outlines the imaging region. $\mathrm{C}$ : Cross sectional overlaid PAI images at the two white dashed lines in (B). GP, gelatin pad; UA, ulnar artery; RA, radial artery; T, tendon; PA, photoacoustic; US, ultrasound; and MAP, maximum amplitude projection. by Kim J et al.(79) licensed under CC BY 4.0

\subsubsection{Clinical application of PA/}

The clinical application of PAI has been investigated in different clinical studies and has been applied as a non-invasive diagnostic technique to assess the microvascularization in different clinical contexts (e.g., breast cancer, skin cancer, port wine stain, synovitis and microvasculature).

Multiple studies have been performed where PAl was used for visualization and diagnosis of breast cancer (80-83). Oraevsky et al. (80) visualized the relative concentration of oxy- and deoxyhaemoglobin in the microvasculature in and around malignant breast tumours, using $757 \mathrm{~nm}$ and $1064 \mathrm{~nm}$ laser light. They demonstrated that PAI bears potential for tumour differentiation. However, those results should be interpreted with caution because of methodological shortcomings, such as not mentioning the number of included patients. Manohar et al. (81) used PAI to visualize breast cancer in vivo at a single wavelength (1064 nm). They performed PAl scans in 13 breast cancer patients and presented two cases in their paper. They found that PAI has potential to visualize breast cancer. This potential of PAI in the clinical setting of cancer is further shown in later studies $(82,83)$. The results of a clinical study among 27 skin cancer patients, for example, showed that PAI was able to detect 90\% (18/20) of the malignant lesions (82). Another study by Heijblom et al. (83) confirmed those findings and demonstrated the potential value of PAl for the imaging of breast tumors. In this study they described the results of 12 new patient measurements for breast cancer analysis and were able to discriminate between breast tumours and cysts. Furthermore, they expected that the potential of PAI could be further improved, when better contrast and imaging depth could be obtained by using better wavelengths or when the image configuration would be changed. 
Another clinical application of PAl is in the field of skin cancer. Zhou et al. (84) performed an animal study with mouse models. They were able to successfully use PAI to detect the tumour depth in vivo till a depth of $3.75 \mathrm{~mm}$ in nude mice. In a later study of Chuah et al. (85) similar results were found when they performed PAI measurements on three patients with skins tumours. They used PAI to acquire a 3D image of the skin tumours and compared those measurements with histological measurements. A good positive correlation between those measurements and the histology was found. Finally, Attia et al. (86) performed a study among 21 skin cancer patients. They were able to discriminate between tumour and healthy skin. Also, they created a 3D image with a spatial resolution of $80 \mu \mathrm{m}$ and found a good correlation $(\mathrm{ICC}=0.81)$ between this $3 \mathrm{D}$ image and the histological measurements.

PAI has also been used in the context of port wine stain birthmarks. In a study by Viator et al. (87), a self-designed and constructed photoacoustic probe was used to determine their depth in tissue phantoms and in one human subject with a port wine stain on his left upper extremity. They found that the photoacoustic probe determined depth in human skin to $570 \mu \mathrm{m}$ and that the acoustic signals had a high signal-to-noise ratio, indicating that PAI could be feasible to probe human skin deeper than a millimetre, sufficient for most port wine stains.

The feasibility of detection of synovitis in finger joints in rheumatoid arthritis patients with PAI was tested by Van den Berg et al. (88) using a handheld ultrasound probe with integrated diode lasers (Figure 7). They used a portable system to examine inflamed and non-inflamed proximal interphalangeal joints of ten patients and compared these with joints from seven healthy subjects. Next to the PAI scans, they also performed ultrasound power Doppler in order to compare the results. They found that PAI was sensitive to clinically evident synovitis: PAI response was four to tenfold higher in inflamed joints compared with contralateral non-inflamed joints and with joints from healthy subjects ( $p<0.001$ for both comparisons). Furthermore, they found that ultrasound power Doppler and PAI were strongly correlated (Spearman's $r=0.64$, with $95 \% \mathrm{Cl}: 0.42,0.79$ ), and concluded that PAl is a promising technique for diagnosis and monitoring of inflammation in peripheral joints. 


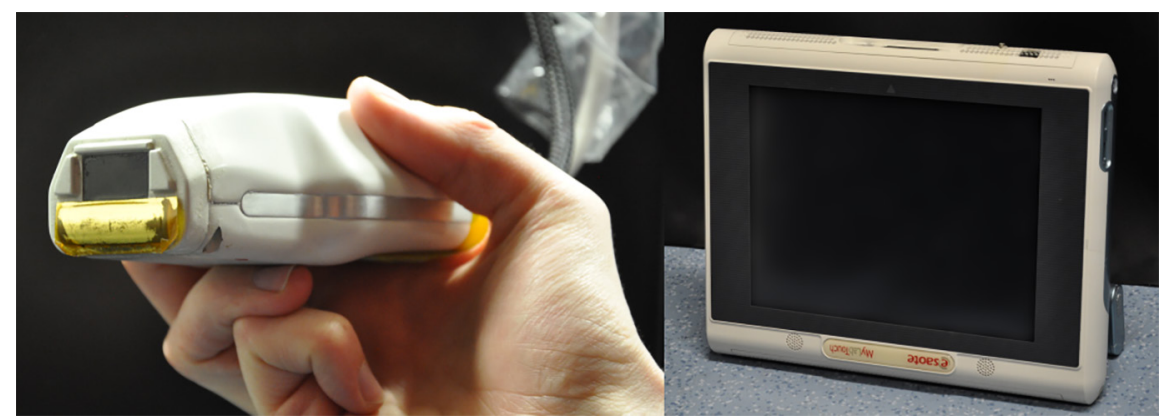

Figure 7: PAl device (handheld PAl probe), as used in the study of Van den Berg et al. (88). Source: Mennes, O.A., 2018. PAI device. [photograph] (personal collection).

A study by Favazza et al. (89) showed the possibilities of PAI to measure cutaneous microvasculature in human skin. They performed measurements on the palms of healthy volunteers, and were able to inflict and measure the hemodynamic response to occlusion tests to simulate the effect of ischemia in the skin. A decrease in the relative oxygen saturation during those occlusions was found and an increase in saturation during the post occlusive hyperaemia reaction. Another study from Taruttis et al. (90) showed the possibility of measuring the vasculature of the human foot with a handheld PAI probe. In this study they included ten healthy volunteers and measured the arteries and microvascular structures of the feet. They were able to measure the larger arteries (e.g. tibialis posterior and dorsalis pedis) as well as smaller blood vessels with a diameter of less than $100 \mu \mathrm{m}$. Furthermore, based on the measured difference in the oxygenation of blood, they were able to distinguish between arteries and veins and could even monitor the arterial pulse. Taruttis et al. (90) concluded that PAl could be a promising technique for the vascular imaging applications. Although measurements in both studies were performed in healthy volunteers, the results might be an indication for the potential of PAI to measure the levels of ischemia in diabetic foot patients.

\subsubsection{PA/ and the diabetic foot}

Our full text search with the combined search terms of "photoacoustic" or "optoacoustic" in combination with "diabetic foot" and "vascularization" resulted in zero hits. In the previous paragraph we described the application of PAI in various clinical settings, with some studies suggesting it might be a promising technique to measure vascularization, also in feet. Keeping in mind that the microcirculatory assessment for the diabetic foot population differs from other vascular disease areas, as mentioned in the introduction of this paper (15), we conclude that research about the possibilities of PAl in the measurement of microvascularization in the diabetic foot is warranted. 


\subsection{DISCUSSION}

This review provided an overview of novel optical imaging techniques that can be used as a replacement or complementary to the currently used blood pressure measurements for the diagnostic assessment of microcirculation in people with a diabetic foot ulcer (Table 1). We focused on Laser Speckle based Perfusion Imaging (LDPI and LSCI), Hyperspectral Imaging (HSI) and Photoacoustic Imaging (PAI). Each of these techniques has specific advantages and disadvantages, and potential use in the clinic depends on the aims of the measurements, as well as the clinical relevance. However, none of the techniques has a clearly established clinical relevance yet, with a only a limited number of publications found describing some clinical diabetic foot ulcer-related outcomes (Table 2). It needs to be determined in future research which technique is the most clinically relevant for the assessment of the microcirculation in diabetic foot ulcers. With this review, more insight is gained about the pros and cons of the novel optical imaging techniques. These are helpful for clinicians and researchers to decide which technique can be used for their purpose.

Table 1: Overview of characteristics of novel imaging techniques (Laser Doppler Perfusion Imaging (LDPI), Laser Speckle Contrast Imaging (LSCl), Hyperspectral Imaging (HSI) and Photoacoustic Imaging (PAI)

\begin{tabular}{|c|c|c|c|c|c|}
\hline Technique & $\begin{array}{l}\text { Physiological } \\
\text { measurement }\end{array}$ & $\begin{array}{l}\text { Penetration depth / } \\
\text { Imaging range }\end{array}$ & Wavelength & $\begin{array}{l}\text { Frame } \\
\text { rate }\end{array}$ & $\begin{array}{l}\text { Additional } \\
\text { characteristics }\end{array}$ \\
\hline LDPI & $\begin{array}{l}\text { Movement of } \\
\text { blood in micro- } \\
\text { circulation }\end{array}$ & $\begin{array}{l}100 \text { to } 1500 \mathrm{um} / \\
50 \times 50 \mathrm{~cm}^{2}\end{array}$ & $633-785 \mathrm{~nm}$ & $\begin{array}{l}\text { Up to } \\
1 \mathrm{fps}\end{array}$ & $\begin{array}{l}\text { 2D imaging; } \\
\text { compact device; } \\
\text { non-contact } \\
\text { measurement }\end{array}$ \\
\hline $\mathrm{LSCl}$ & $\begin{array}{l}\text { Movement of } \\
\text { blood in } \\
\text { microcirculation }\end{array}$ & $\begin{array}{l}100 \text { to } 500 \mathrm{um} / \\
24 \times 24 \mathrm{~cm}^{2}\end{array}$ & $785 \mathrm{~nm}$ & $\begin{array}{l}\text { Up to } \\
100 \mathrm{fps}\end{array}$ & $\begin{array}{l}\text { 2D imaging; } \\
\text { compact device; } \\
\text { non-contact } \\
\text { measurement }\end{array}$ \\
\hline $\mathrm{HSI}$ & $\begin{array}{l}\text { Absorption } \\
\text { spectrum of } \\
\text { tissue, mainly } \\
\text { haemoglobins }\end{array}$ & $\begin{array}{l}1 \text { to } 5 \mathrm{~mm} / \\
50 \times 50 \mathrm{~cm}^{2}\end{array}$ & $200-2500 \mathrm{~nm}$ & $\begin{array}{l}\text { Up to } \\
1000 \\
\text { fps }\end{array}$ & $\begin{array}{l}\text { 2D imaging; } \\
\text { compact device; } \\
\text { non-contact } \\
\text { measurement }\end{array}$ \\
\hline PAI & $\begin{array}{l}\text { Absorption } \\
\text { (spectrum) of } \\
\text { tissue, mainly } \\
\text { haemoglobins }\end{array}$ & $\begin{array}{l}1 \text { to } 100 \mathrm{~mm} / \\
\text { B-scan of } 4 \mathrm{~cm}\end{array}$ & $808-980 \mathrm{~nm}$ & $\begin{array}{l}\text { Up to } \\
50 \mathrm{fps}\end{array}$ & $\begin{array}{l}\text { 2D/3D imaging; } \\
\text { less compact } \\
\text { device; contact } \\
\text { measurement }\end{array}$ \\
\hline
\end{tabular}




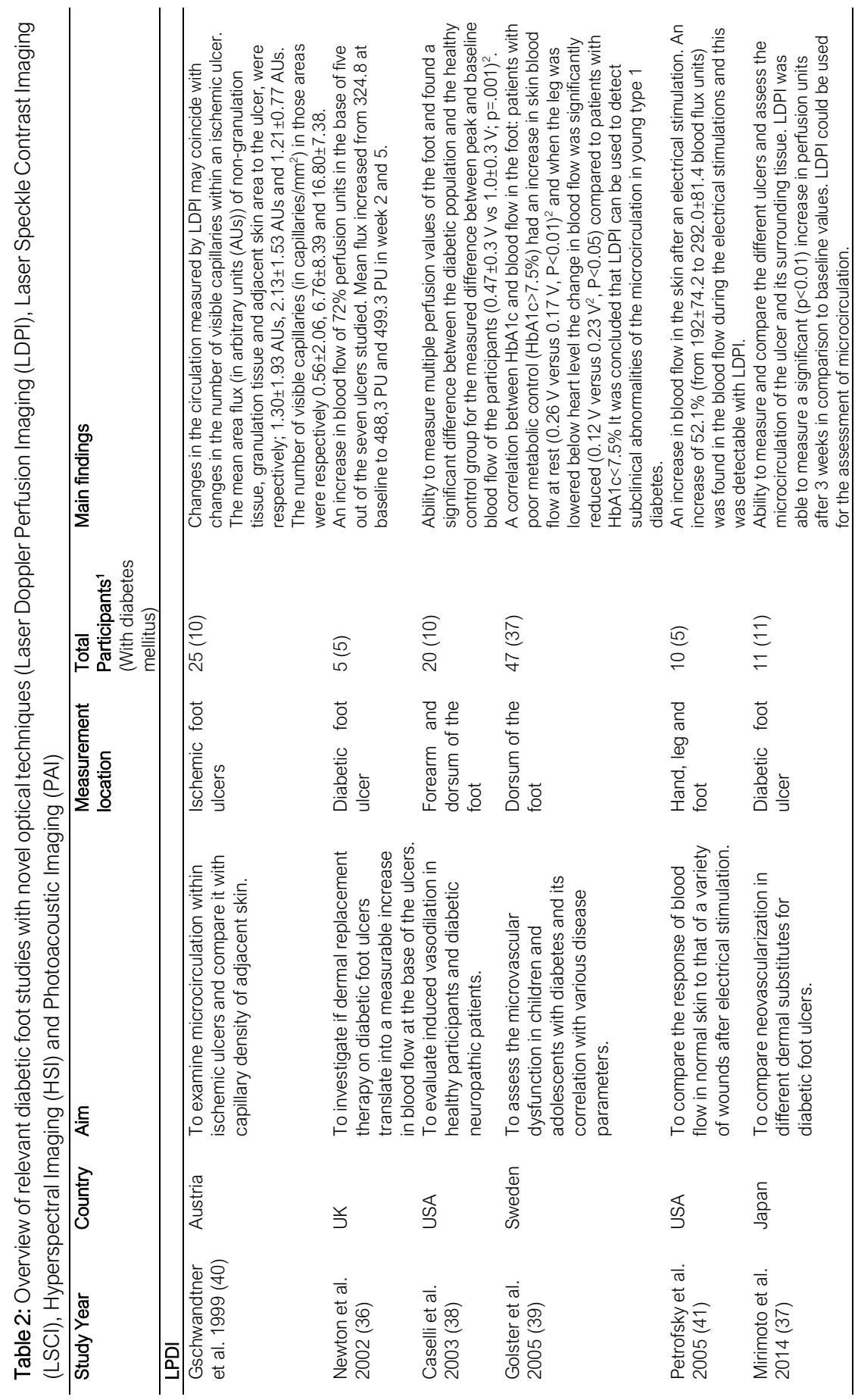




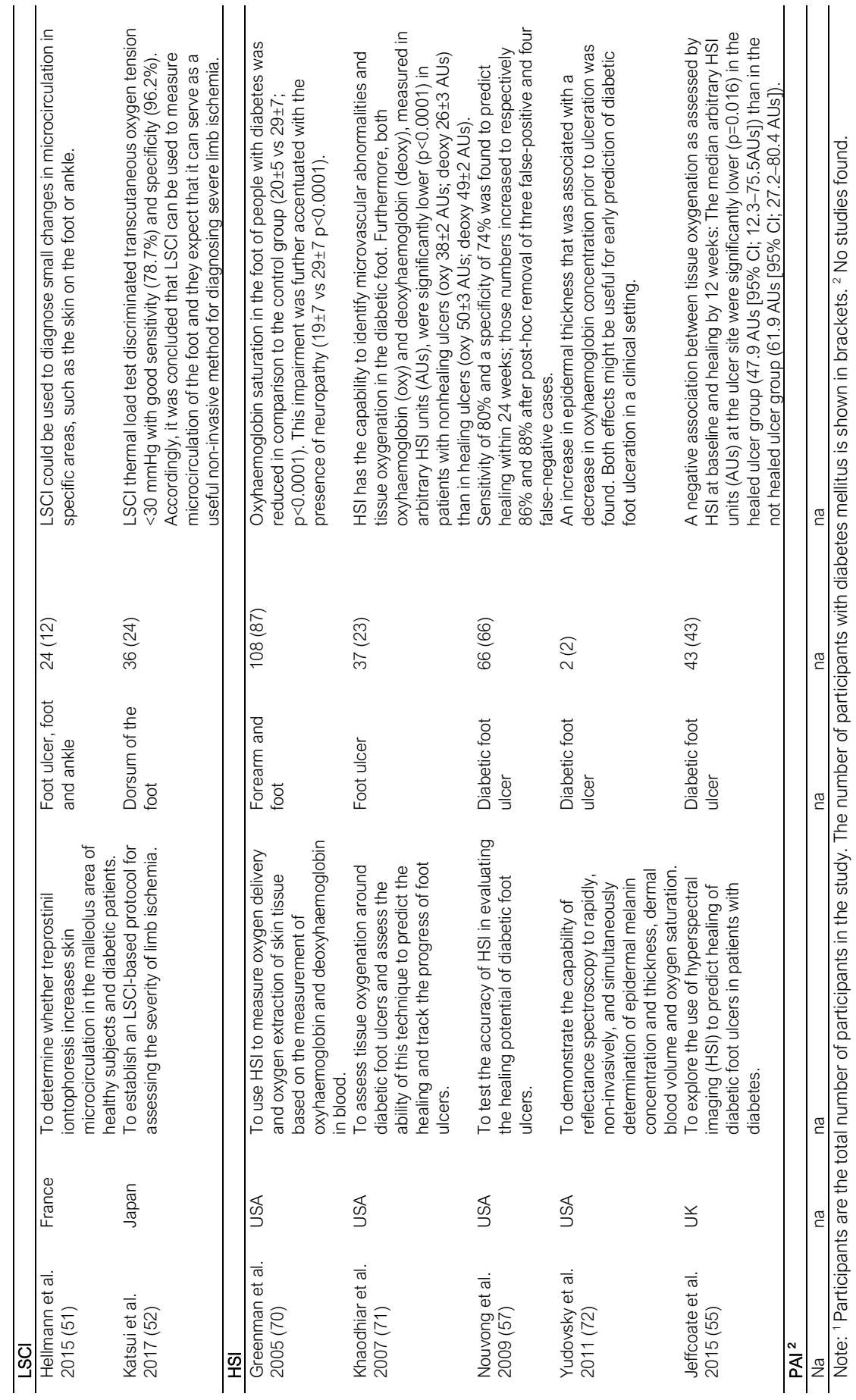


The first important consideration is the technical background of each technique. This determines the aspect of microcirculation that can be captured. If blood flow or blood circulation should be measured, both LDPI and LSCI would be the techniques of choice, as they measure the motion of blood from dynamic speckle patterns (24). For the measurement of the composition of tissue or the (relative) concentration of oxyand deoxyhaemoglobin in blood, HSI or (multiwavelength) PAI should be used. For the measurement of the presence of blood (different from blood flow or blood pressure), both HSI or PAI would be a good choice, as both techniques measure the presence of blood based on its absorption of light, which does not depend on movement in tissue. For 2D imaging in the plane of the skin surface, HSI would be well suited, for 3D imaging PAI is necessary. In comparison with LSCI, LDPI and HSI, PAI is able to deliver 3D images instead of 2D ones. This could be particularly useful in cases when a better overview is necessary, for example before or during revascularization of the foot.

Second, aspects such as costs, usability, and the need for non-contact methods should be considered. Generally, the costs for the equipment for each technique are tens of thousands of euros. Most PAI systems are quite expensive because of the laser, however, these costs can be reduced when a diode laser is used, as was for example applied in the study by van den Berg et al. (88). Furthermore, LDPI systems are more expensive than LSCI because the camera for LDPI needs to have a higher frame-rate to capture the LDPI data.

User friendliness differs among the four optical imaging techniques. PAI systems are usually less compact and therefore less convenient to use in the clinical setting. This might be fixed with the current development of handheld PAI probes, as described in multiple studies $(74,84,86,88,90)$, although the scanning surface and therefore the imaging dimensions are smaller in those devices. LDPI, LSCI and HSI are all available in compact systems and usable on portable trolleys for bedside measurements for the patient.

In the clinical setting of diabetic foot ulcers, it is preferable to use non-contact methods $(16,17)$. Regarding this aspect, PAI is less applicable than the other three systems because PAI needs to have skin-contact in order to detect the returning ultrasound. This is performed using contact gel or water; however, and is not suitable in the context of an open wound, and therefore less desirable in the measurement of diabetic foot ulcers.

We discussed four techniques in this review paper, however, other promising imaging techniques are available that may also be relevant in the diabetic foot ulcers. One example is Infrared thermography, which is currently used in the diagnostics of inflammation and in the prevention of diabetic foot ulcers $(91,92)$. However, it is not yet 
commonly used for the assessment of microcirculation and therefore it was not included in this review.

\subsubsection{Future research}

As outlined above, additional research on LSCI, LDPI, HSI, and PAI for the assessment of microcirculation in the diabetic foot is needed, in both the technical as well in the clinical setting. Some examples of a future research agenda are as follows.

LSCI has great potential as a real-time non-invasive technique. However, the used perfusion units (PU's) are not yet correlated to non-arbitrary units. For example, it is not possible to measure $\mathrm{PU}$ in flow units such as $\mathrm{ml}$ blood $/ \mathrm{min} / \mathrm{mm}^{3}$ tissue. Furthermore, the clinical relevance of $\mathrm{LSCl}$ is unknown. This is also the case for LDPI measurements. Although LDPI is more well-known since it is possible to translate the measured Doppler shift to velocity, the correlation to healing tendency is not known and therefore and improvement can be achieved in this area.

Both $\mathrm{HSI}$ and PAI have major potential when measuring the composition of tissue or concentration of blood, and its oxygen saturation. However, the pathologic consequences based on the different concentration levels of blood are not well known. For example Jeffcoate et al. (53) found a negative association between tissue oxygenation and healing, what is in contrast with studies of Khaodhiar et al. (68) and Nouvong et al. (69), who found a positive association between tissue oxygenation and ulcer healing. More research to the healing tendency of ulcers based on the measured concentrations of oxy- and deoxyhaemoglobin could give more insight in the healing process of wounds and the prediction of ulcer healing.

For PAI there are zero publications where PAI is used to measure the microcirculation for the diabetic foot. Only a few small clinical studies are available that are applicable for the diabetic foot population. It is therefore interesting to look further into the usefulness of PAI in this field. One advantage of PAl is that it can conduct 3D-imaging. It would be of interest to examine the possibilities of this feature for the diabetic foot, because of better delineation of the affected bone and soft tissue involvement, beside the vascular blood compartment evaluation.

\subsection{CONCLUSION}

In this review, four novel optical techniques for assessment of microcirculation in people with a diabetic foot ulcer were evaluated. All of them seem promising for the measurement of various aspects of the microcirculation in and around a diabetic foot ulcer. However, none of the techniques has been sufficiently tested in a clinical setting 
with people with diabetic foot ulcers, and as such their added value for the clinic is currently still uncertain. Although the possibilities are promising, the current novel optical imaging techniques need to be technically improved and prospective trials are necessary to evaluate their clinical value. 


\section{REFERENCES}

1. Alexiadou K, Doupis J. Management of diabetic foot ulcers. Diabetes Ther. 2012;3:115.

2. Kerr M, Rayman G, Jeffcoate WJ. Cost of diabetic foot disease to the National Health Service in England. Diabet Med. 2014 Dec;31:1498-504.

3. Prompers L, Huijberts M, Schaper N, Apelqvist J, Bakker K, Edmonds M, et al. Resource utilisation and costs associated with the treatment of diabetic foot ulcers. Prospective data from the Eurodiale Study. Diabetologia. 2008;51:1826-34.

4. Prompers L, Schaper N, Apelqvist J, Edmonds M, Jude E, Mauricio D, et al. Prediction of outcome in individuals with diabetic foot ulcers: Focus on the differences between individuals with and without peripheral arterial disease. The EURODIALE Study. Diabetologia. 2008;51:747-55.

5. Bakker K, Apelqvist J, Lipsky BA, Van Netten JJ, Schaper NC. The 2015 IWGDF guidance documents on prevention and management of foot problems in diabetes: development of an evidence-based global consensus. Diabetes Metab Res Rev. 2015 Sep 27;

6. Hinchliffe R, Brownrigg J, Apelqvist J, Boyko EJ, Fitridge R, Mills J, et al. IWGDF guidance on the diagnosis, prognosis and management of peripheral artery disease in patients with foot ulcers in diabetes. Diabetes Metab Res Rev. 2016;32 Suppl 1:37-44.

7. Armstrong DG, Boulton AJM, Bus SA. Diabetic Foot Ulcers and Their Recurrence. N Engl J Med. 2017;376:2367-75.

8. Narres M, Kvitkina T, Claessen H, Droste S, Schuster B, Morbach S, et al. Incidence of lower extremity amputations in the diabetic compared with the non-diabetic population: A systematic review. Vol. 12, PLoS ONE. 2017.

9. Skrepnek GH, Mills JL, Lavery LA, Armstrong DG. Health care service and outcomes among an estimated 6.7 million ambulatory care diabetic foot cases in the U.S. Diabetes Care. 2017 Jul;40:936-42.

10. Aldworth J, Bache N Al, Hegelund MH, Hirst SM, Linnenkamp U, Magliano D, et al. IDF Diabetes Atlas 7th edition. 2015. 144 p.

11. Brownrigg JRW, Hinchliffe RJ, Apelqvist J, Boyko EJ, Fitridge R, Mills JL, et al. Effectiveness of bedside investigations to diagnose peripheral artery disease among people with diabetes mellitus: a systematic review. Diabetes Metab Res Rev. 2016 Jan;32:119-27.

12. Brownrigg JRW, Hinchliffe RJ, Apelqvist J, Boyko EJ, Fitridge R, Mills JL, et al. Performance of prognostic markers in the prediction of wound healing or amputation among patients with foot ulcers in diabetes: A systematic review. Diabetes Metab Res Rev. 2016 Jan;32:128-35.

13. Sumpio BE, Forsythe RO, Ziegler KR, Van Baal JG, Lepantalo MJA, Hinchliffe RJ. Clinical implications of the angiosome model in peripheral vascular disease. J Vasc Surg. 2013;58:814-26.

14. Elgzyri T, Larsson J, Thörne J, Eriksson KF, Apelqvist J. Outcome of ischemic foot ulcer in diabetic patients who had no invasive vascular intervention. Eur J Vasc Endovasc Surg. 2013;46:110-7.

15. Boulton AJM, Scarpello JHB, Ward JD. Venous oxygenation in the diabetic neuropathic foot: Evidence of arteriovenous shunting? Diabetologia. 1982 Jan;22:6-8.

16. Allen J, Howell K. Microvascular imaging: Techniques and opportunities for clinical physiological measurements. Physiol Meas. 2014;35. 
17. Forsythe RO, Hinchliffe RJ. Assessment of foot perfusion in patients with a diabetic foot ulcer. Diabetes Metab Res Rev. 2016;32:232-8.

18. Leahy MJ, Enfield JG, Clancy NT, O'Doherty J, McNamara P, Nilsson GE. Biophotonic methods in microcirculation imaging. Med Laser Appl. 2007;22:105-26.

19. Sakr Y. Techniques to assess tissue oxygenation in the clinical setting. Transfus Apher Sci. 2010;43:79-94.

20. Sowa MG, Kuo W-C, Ko AC-T, Armstrong DG. Review of near-infrared methods for wound assessment. J Biomed Opt. 2016;21:91304.

21. Arksey H, O'Malley L. Scoping studies: Towards a methodological framework. Int J Soc Res Methodol Theory Pract. 2005 Feb;8:19-32.

22. Nilsson GE, Tenland T, Oberg PA. Evaluation of a Laser Doppler Flowmeter for Measurement of Tissue Blood Flow. IEEE Trans Biomed Eng. 1980 Oct;BME-27:597604.

23. Stern MD. In vivo evaluation of microcirculation by coherent light scattering. Nature. 1975 Mar 6;254:56-8.

24. Briers D. Laser Doppler, speckle and related techniques for blood perfusion mapping and imagingLaser Doppler, speckle and related techniques for blood perfusion mapping and imaging. 2001;35.

25. Briers JD. Laser speckle contrast imaging for measuring blood flow. 2007;XXXVII:14.

26. Leutenegger M, Martin-Williams E, Harbi P, Thacher T, Raffoul W, André M, et al. Realtime full field laser Doppler imaging. Biomed Opt Express. 2011 Jun 1;2:1470-7.

27. Draijer M, Hondebrink E, Van Leeuwen T, Steenbergen W. Review of laser speckle contrast techniques for visualizing tissue perfusion. Lasers Med Sci. 2009;24:639-51.

28. Bezemer R, Klijn E, Khalilzada M, Lima A, Heger M, van Bommel J, et al. Validation of near-infrared laser speckle imaging for assessing microvascular (re)perfusion. Microvasc Res. 2010;79:139-43.

29. Hoeksema H, Van de Sijpe K, Tondu T, Hamdi M, Van Landuyt K, Blondeel P, et al. Accuracy of early burn depth assessment by laser Doppler imaging on different days post burn. Burns. 2009;35:36-45.

30. Pape SA, Baker RD, Wilson D, Hoeksema H, Jeng JC, Spence RJ, et al. Burn wound healing time assessed by laser Doppler imaging (LDI). Part 1: Derivation of a dedicated colour code for image interpretation. Burns. 2012;38:187-94.

31. Pape SA, Baker RD, Wilson D, Hoeksema H, Jeng JC, Spence RJ, et al. Burn wound healing time assessed by laser Doppler imaging (LDI). Part 1: Derivation of a dedicated colour code for image interpretation. Burns. 2012;38:187-94.

32. Anderson ME, Moore TL, Lunt M, Herrick AL. Digital iontophoresis of vasoactive subtances as measured by laser Doppler imaging - A non-invasive technique by which to measure microvascular dysfunction in Raynaud's phenomenon. Rheumatology. 2004 Aug 1;43:986-91.

33. Hughes M, Moore T, Manning J, Wilkinson J, Dinsdale G, Roberts C, et al. Reduced perfusion in systemic sclerosis digital ulcers (both fingertip and extensor) can be increased by topical application of glyceryl trinitrate. Microvasc Res. 2017;111:32-6.

34. Ljung P, Bornmyr S, Svensson $\mathrm{H}$. Wound healing after total elbow replacement in rheumatoid arthritis wound complications in 50 cases and laser-doppler imaging of skin microcirculation. Acta Orthop. 1995 Jan 8;66:59-63. 
35. Eichhorn W, Auer T, Voy ED, Hoffmann K. Laser Doppler imaging of axial and random pattern flaps in the maxillo-facial area. A preliminary report. J Cranio-Maxillofacial Surg. 1994 Oct;22:301-6.

36. Newton DJ, Khan F, Belch JJF, Mitchell MR, Leese GP. Blood flow changes in diabetic foot ulcers treated with dermal replacement therapy. J Foot Ankle Surg. 2002 Jul;41:233-7.

37. Morimoto N, Kakudo N, Valentin Notodihardjo P, Suzuki S, Kusumoto K. Comparison of neovascularization in dermal substitutes seeded with autologous fibroblasts or impregnated with bFGF applied to diabetic foot ulcers using laser Doppler imaging. J Artif Organs. 2014 Dec 16;17:352-7.

38. Caselli A, Hanane T, Jane B, Carter S, Khaodhiar L, Veves A. Topical methyl nicotinateinduced skin vasodilation in diabetic neuropathy. J Diabetes Complications. 2003;17:205-10.

39. Golster H, Hyllienmark L, Ledin T, Ludvigsson J, Sjoberg F. Impaired microvascular function related to poor metabolic control in young patients with diabetes. ClinPhysiol Functlmaging. 2005;25:100-5.

40. Gschwandtner ME, Ambrózy E, Schneider B, Fasching S, Willfort A, Ehringer H. Laser doppler imaging and capillary microscopy in ischemic ulcers. Atherosclerosis. 1999 Jan 3;142:225-32.

41. Petrofsky J, Schwab E, Lo T, Cúneo M, George J, Kim J, et al. Effects of electrical stimulation on skin blood flow in controls and in and around stage III and IV wounds in hairy and non hairy skin. Med Sci Monit. 2005;11:CR309-16.

42. Mirdell R, Iredahl F, Sjöberg F, Farnebo S, Tesselaar E. Microvascular blood flow in scalds in children and its relation to duration of wound healing: A study using laser speckle contrast imaging. Burns. 2016;42:648-54.

43. van Vuuren TMAJ, Van Zandvoort C, Doganci S, Zwiers I, tenCate-Hoek AJ, Kurstjens RLM, et al. Prediction of venous wound healing with laser speckle imaging. Phlebology. 2017;32:658-64.

44. Hecht N, Müller M-M, Sandow N, Pinczolits A, Vajkoczy P, Woitzik J. Infarct prediction by intraoperative laser speckle imaging in patients with malignant hemispheric stroke. J Cereb Blood Flow Metab. 2016;36:1022-32.

45. Bezemer R, Legrand M, Klijn E, Heger M, Post ICJH, van Gulik TM, et al. Real-time assessment of renal cortical microvascular perfusion heterogeneities using nearinfrared laser speckle imaging. Opt Express. 2010;18:15054-61.

46. Dunn AK. Laser speckle contrast imaging of cerebral blood flow. Ann Biomed Eng. 2012;40:367-77.

47. Shiga Y, Kunikata H, Aizawa N, Kiyota N, Maiya Y, Yokoyama Y, et al. Optic Nerve Head Blood Flow, as Measured by Laser Speckle Flowgraphy, Is Significantly Reduced in Preperimetric Glaucoma. Curr Eye Res. 2016;41:1447-53.

48. Humeau-Heurtier A, Guerreschi E, Abraham P, Mahe G. Relevance of laser doppler and laser speckle techniques for assessing vascular function: State of the art and future trends. IEEE Trans Biomed Eng. 2013;60:659-66.

49. Roustit M, Cracowski JL. Assessment of endothelial and neurovascular function in human skin microcirculation. Trends Pharmacol Sci. 2013;34:373-84.

50. Zhang S, Gnyawali S, Huang J, Ren W, Gordillo G, Sen CK, et al. Multimodal imaging of cutaneous wound tissue. J Biomed Opt. 2015;20:16016. 
51. Hellmann M, Roustit M, Gaillard-Bigot F, Cracowski JL. Cutaneous iontophoresis of treprostinil, a prostacyclin analog, increases microvascular blood flux in diabetic malleolus area. Eur J Pharmacol. 2015;758:123-8.

52. Katsui S, Inoue Y, Igari K, Toyofuku T, Kudo T, Uetake H. Novel assessment tool based on laser speckle contrast imaging to diagnose severe ischemia in the lower limb for patients with peripheral arterial disease. Lasers Surg Med. 2017;49:645-51.

53. Yudovsky D, Nouvong A, Pilon L. Hyperspectral imaging in diabetic foot wound care. J Diabetes Sci Technol. 2010 Sep;4:1099-113.

54. Gould LJ. Technology: Noninvasive Assessment of Lower Extremity Healing Potential. Foot Ankle Spec. 2008 Apr;1:115-6.

55. Jeffcoate WJ, Clark DJ, Savic N, Rodmell PI, Hinchliffe RJ, Musgrove A, et al. Use of $\mathrm{HSI}$ to measure oxygen saturation in the lower limb and its correlation with healing of foot ulcers in diabetes. Diabet Med. 2015;32:798-802.

56. Tuchin VV. Tissue optics: light scattering methods and instruments for medical diagnosis. SPIE Pres. 2007. 3-142 p.

57. Nouvong A, Hoogwerf B, Mohler E, Davis B, Tajaddini A, Medenilla E. Evaluation of diabetic foot ulcer healing with hyperspectral imaging of oxyhemoglobin and deoxyhemoglobin. Diabetes Care. 2009 Nov 1;32:2056-61.

58. Lu G, Fei B. Medical hyperspectral imaging: a review. J Biomed Opt. 2014 Jan 20;19:10901.

59. Ferris DG, Lawhead R a, Dickman ED, Holtzapple N, Miller J a, Grogan S, et al. Multimodal hyperspectral imaging for the noninvasive diagnosis of cervical neoplasia. J Low Genit Tract Dis. 2001;5:65-72.

60. Pap Smear- ClinicalKey https://www.clinicalkey.com/\#!/content/medical_procedure/19-s2.0-mp_FM-020.

61. Wang C, Zheng W, Bu Y, Chang S, Zhang S, Xu RX. Multi-scale hyperspectral imaging of cervical neoplasia. Arch Gynecol Obstet. 2015;293:1309-17.

62. Panasyuk S V., Yang S, Faller D V., Ngo D, Lew RA, Freeman JE, et al. Medical hyperspectral imaging to facilitate residual tumor identification during surgery. Cancer Biol Ther. 2007;6:439-46.

63. Akbari H, Halig L V., Schuster DM, Osunkoya A, Master V, Nieh PT, et al. Hyperspectral imaging and quantitative analysis for prostate cancer detection. J Biomed Opt. 2012;17:760051.

64. Kong SG, Martin M, Vo-Dinh T. Hyperspectral Fluorescence Imaging for Mouse Skin Tumor Detection. ETRI J. 2006 Dec 7;28:770-6.

65. Gillies R, Freeman JE, Cancio LC, Brand D, Hopmeier M, Mansfield JR. Systemic Effects of Shock and Resuscitation Monitored by Visible Hyperspectral Imaging. Diabetes Technol Ther. 2003 Oct;5:847-55.

66. Cancio LC, Batchinsky Al, Mansfield JR, Panasyuk S, Hetz K, Martini D, et al. Hyperspectral Imaging: A New Approach to the Diagnosis of Hemorrhagic Shock. J Trauma Inj Infect Crit Care. 2006 May;60:1087-95.

67. Mordant DJ, Al-Abboud I, Muyo G, Gorman A, Sallam A, Ritchie P, et al. Spectral imaging of the retina. Eye. 2011 Mar 10;25:309-20.

68. Neville R, Gupta S. Establishment of normative perfusion values using hyperspectral tissue oxygenation mapping technology. 2009 Nov;6:156-61. 
69. Chin JA, Wang EC, Kibbe MR. Evaluation of hyperspectral technology for assessing the presence and severity of peripheral artery disease. J Vasc Surg. 2011 Dec;54:167988.

70. Greenman RL, Panasyuk S, Wang X, Lyons TE, Dinh T, Longoria L, et al. Early changes in the skin microcirculation and muscle metabolism of the diabetic foot. Lancet. 2005;366:1711-7.

71. Khaodhiar L, Dinh T, Schomacker K, Panasyuk S V., Freeman JE, Lew R, et al. The Use of Medical Hyperspectral Imaging (MHSI) To Evaluate Microcirculatory Changes in Diabetic Foot Ulcers and Predict Clinical Outcomes. Diabetes Care. 2007;30:903-10.

72. Yudovsky D, Nouvong A, Schomacker K, Pilon L. Monitoring temporal development and healing of diabetic foot ulceration using hyperspectral imaging. J Biophotonics. 2011;4:565-76.

73. Xia J, Yao J, Wang L V. Photoacoustic tomography: principles and advances. Electromagn waves (Cambridge, Mass). Jan;147:1-22.

74. Gujrati V, Mishra A, Ntziachristos V. Molecular imaging probes for multi-spectral optoacoustic tomography. Chem Commun. 2017;53:4653-72.

75. Lee HY, Hyun SH, Lee KS, Kim B-T, Kim J, Shim YM, et al. Volume-based parameter of 18)F-FDG PET/CT in malignant pleural mesothelioma: prediction of therapeutic response and prognostic implications. Ann Surg Oncol. 2010 Oct;17:2787-94.

76. Yang X, Stein EW, Ashkenazi S, Wang L V. Nanoparticles for photoacoustic imaging. Wiley Interdiscip Rev Nanomedicine Nanobiotechnology. 2009 Jul 1;1:360-8.

77. Luke GP, Yeager D, Emelianov SY. Biomedical applications of photoacoustic imaging with exogenous contrast agents. Ann Biomed Eng. 2012 Feb 3;40:422-37.

78. Zhang Y, Yu J, Kahkoska AR, Gu Z. Photoacoustic drug delivery. Vol. 17, Sensors (Switzerland). Multidisciplinary Digital Publishing Institute; 2017. p. 1400.

79. Kim J, Park S, Jung Y, Chang S, Park J, Zhang Y, et al. Programmable Real-time Clinical Photoacoustic and Ultrasound Imaging System. Sci Rep. 2016 Dec 12;6:35137.

80. Oraevsky AA, Savateeva E V., Solomatin S V., Karabutov AA, Andreev VG, Gatalica Z, et al. Optoacoustic imaging of blood for visualization and diagnostics of breast cancer. 2002;81-94.

81. Manohar S, Vaartjes SE, van Hespen JCG, Klaase JM, van den Engh FM, Steenbergen $W$, et al. Initial results of in vivo non-invasive cancer imaging in the human breast using near-infrared photoacoustics. Opt Express. 2007;15:12277.

82. Ermilov SA, Khamapirad T, Conjusteau A, Leonard MH, Lacewell R, Mehta K, et al. Laser optoacoustic imaging system for detection of breast cancer. J Biomed Opt. 2009;14:24007.

83. Heijblom M, Piras D, Xia W, van Hespen JCG, Klaase JM, van den Engh FM, et al. Visualizing breast cancer using the Twente photoacoustic mammoscope: What do we learn from twelve new patient measurements? Opt Express. 2012;20:11582.

84. Zhou Y, Xing W, Maslov KI, Cornelius LA, Wang L V. Handheld photoacoustic microscopy to detect melanoma depth in vivo. Opt Lett. 2014;39:4731.

85. Chuah SY, Attia ABE, Long V, Ho CJH, Malempati P, Fu CY, et al. Structural and functional 3D mapping of skin tumours with non-invasive multispectral optoacoustic tomography. Ski Res Technol. 2017;23:221-6.

86. Attia ABE, Chuah SY, Razansky D, Ho CJH, Malempati P, Dinish US, et al. Noninvasive real-time characterization of non-melanoma skin cancers with handheld optoacoustic probes. Photoacoustics. 2017;7:20-6. 
87. Viator JA, Au G, Paltauf G, Jacques SL, Prahl SA, Ren H, et al. Clinical testing of a photoacoustic probe for port wine stain depth determination. Lasers Surg Med. 2002;30:141-8.

88. van den Berg PJ, Daoudi K, Bernelot Moens HJ, Steenbergen W. Feasibility of photoacoustic/ultrasound imaging of synovitis in finger joints using a point-of-care system. Photoacoustics. 2017;8:8-14.

89. Favazza CP, Cornelius LA, Wang L V. In vivo functional photoacoustic microscopy of cutaneous microvasculature in human skin. J Biomed Opt. 2011;16:26004.

90. Taruttis A, Timmermans AC, Wouters PC, Kacprowicz M, van Dam GM, Ntziachristos V. Optoacoustic Imaging of Human Vasculature: Feasibility by Using a Handheld Probe. Radiology. 2016;281:256-63.

91. Sousa P, Felizardo V, Oliveira D, Couto R, Garcia NM. A review of thermal methods and technologies for diabetic foot assessment. Expert Rev Med Devices. 2015;12:43948.

92. Hernandez-Contreras D, Peregrina-Barreto H, Rangel-Magdaleno J, Gonzalez-Bernal J. Narrative review: Diabetic foot and infrared thermography. Infrared Phys Technol. 2016;78:105-17. 


\section{APPENDIX I}

Database

Scopus (www.scopus.com)

Laser Doppler Perfusion Imaging (Title. Abstract and Keywords)

TITLE-ABS-KEY ( ( laser AND doppler ) AND ( diabetic AND foot ) AND ( circulation OR microcirculation ) ) AND ( LIMIT-TO ( DOCTYPE, "ar " ) OR LIMIT-TO ( DOCTYPE , " re " ) ) AND ( LIMIT-TO ( LANGUAGE , "English " ) )

Laser Speckle Contrast Imaging (Full text)

ALL ( ( laser AND speckle ) AND ( diabetic AND foot ) AND ( circulation OR microcirculation )) AND ( LIMIT-TO ( DOCTYPE , "ar " ) OR LIMIT-TO ( DOCTYPE , " re " ) ) AND ( LIMIT-TO ( LANGUAGE , "English " ) )

Hyperspectral Imaging (Full text)

ALL ( ( hyperspectral ) AND ( diabetic AND foot ) AND ( circulation OR microcirculation) ) AND ( LIMIT-TO ( DOCTYPE , "ar " ) OR LIMIT-TO ( DOCTYPE , " re " ) ) AND ( LIMIT-TO ( LANGUAGE , "English " ) )

Photoacoustic Imaging (Full text)

ALL ( ( photoacoustic OR optoacoustic ) AND ( diabetic AND foot ) AND (vascularization ) ) AND ( LIMIT-TO ( DOCTYPE , "ar ") OR LIMIT-TO ( DOCTYPE , " re " ) ) AND ( LIMIT-TO ( LANGUAGE , "English " )) 



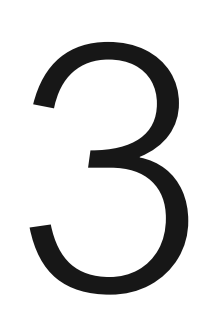

\section{Assessment of Microcirculation in the Diabetic Foot with Laser Speckle Contrast Imaging}

O.A. Mennes, J.J. van Netten, J.G. van Baal, W. Steenbergen.

Physiological Measurement 2019; 40(6): 065002

(DOI: 10.1088/1361-6579/ab2058)

(C) IOP Publishing. Reproduced with permission. All rights reserved. 


\section{ABSTRACT}

Background: A major challenge for treating diabetic foot ulcers is estimating the severity of ischemia, as currently used non-invasive diagnostic techniques provide relatively poor prognostic values. Laser Speckle Contrast Imaging (LSCI) is a promising non-invasive technique to assess microcirculation. Our aim was to investigate the stability and reproducibility of $\mathrm{LSCl}$ for the assessment of microcirculation in the diabetic foot, the relation of LSCI results to currently used non-invasive blood pressure measurements, and the ability of $\mathrm{LSCl}$ to discriminate between the degrees of ischemia.

Method: Thirty-three participants with diabetic foot ulcers were included in this prospective, single centre, observational cohort study that was conducted in the Netherlands. They were classified as non-ischemic, ischemic or critical-ischemic based on criteria formulated in the international guidelines. Two clinicians performed LSCI scans of the foot, consisting of baseline measurements, followed by two stress tests (post-occlusion peak and elevation test). With 3 measurement conditions and 5 regions of interest of the foot per patient, a total of 15 measurements were available for analyses.

Results: The intra-observer agreement of LSCl was high (Interclass Correlation Coefficient $($ ICC $)=0.711-0.950 ; p<0.001)$ for all 15 measurements. The inter-observer agreement was high (ICC $=0.728-0.861 ; p \leq 0.001)$ for 10 measurements and moderate (ICC=0.476-0.570; $p \leq 0.005)$ for the remaining 5 measurements. The inter-assessor agreement was high and significant (ICC $=0.857-0.996 ; \quad p \leq 0.001)$ for all measurements. Correlation between $\mathrm{LSCl}$ and non-invasive blood pressure measurements was low (ICC=-0.272-0.582). During both stress tests, microcirculation was significantly lower in critical-ischemic feet compared to non-ischemic feet $(67.5$ Perfusion Units (PU) vs. 96.3PU and 41.0PU vs. 63.9PU; $p<0.05$ ).

Conclusion: $\mathrm{LSCl}$ is a stable and reproducible technique for assessment of microcirculation in people with diabetic foot ulcers and shows significant differences between non-ischemic, ischemic and critical-ischemic patient populations. 


\subsection{INTRODUCTION}

One of the major complications of diabetes mellitus are diabetic foot ulcers, with an annual prevalence around 2\% and a life-time incidence between 19\% and 34\% (1-4). Diabetic foot ulcers have a high morbidity and mortality, resulting in increasing healthcare costs (5-8) and are an important reason to perform lower extremity amputation, especially in the presence of ischemia (5).

Early and accurate diagnosis is the first step in ischemia treatment and outcome improvement (2). Primary diagnosis of ischemia currently consists of visual inspection and non-invasive assessment of blood pressure in the feet (2). Clinical decisions are based on these measurements; according to international guidelines, urgent vascular imaging and revascularisation are to be considered in patients with a diabetic foot ulcer and either an ankle pressure $<50 \mathrm{mmHg}$ or an ankle-brachial index $(\mathrm{ABI})<0.5$, in patients with a toe pressure $<30 \mathrm{mmHg}$, or in patients with a transcutaneous oxygen pressure $\left(\mathrm{TcpO}_{2}\right)<25 \mathrm{mmHg}(2)$.

However, these techniques only provide relatively poor indications of the severity of ischemia. Furthermore, these values provide relatively poor prognostic values in predicting ulcer healing (9). A suggested explanation is that these measurements are not necessarily reflecting the situation of ischemia at the site of the ulcer. With different angiosomes providing blood supply to the feet, low values in one angiosome may not reflect blood supply at the ulcer location (10). Clinical evidence adds to the poor prognostic values; for example, a large observational cohort study showed some patients with critical ischemia to heal from their foot ulcers without vascular interventions, while some patients with non-ischemic ulcers did not heal despite adequate treatment (11).

Improving assessment of microcirculation in people with diabetic foot ulcers is an important area for research, as identified in international guidelines (2). This may overcome the disadvantages of currently used non-invasive blood pressure measurements (2). An improved diagnostic technique should reflect the situation of ischemia at the ulcer location, be stable (i.e. provide a constant signal over time during the measurements) and reproducible (i.e. have high intra and inter-user correlation), non-invasive, and differ from currently used non-invasive blood pressure measurements.

A potential technique fulfilling these requirements is single-exposure Laser Speckle Contrast Imaging (LSCI). LSCl can measure microcirculation based on changes in the optical speckle pattern generated by motion of red blood cells in the skin or ulcers (12). When skin is illuminated with coherent laser light, the reflected light will create light and 
dark areas, a so-called speckle pattern (12). In perfused skin, moving red blood cells cause a dynamic speckle pattern. The dynamic character of the speckles can be visualized with a camera with an integration time longer than the time scale of the speckle fluctuations, causing the speckle pattern to be blurred on the recorded image (12-14). Increased perfusion (i.e. increased local concentration and/or increased speed of moving red blood cells), result in a reduced spatial speckle contrast $(12,15)$. There is a correlation between the speckle contrast and blood perfusion. By measuring this contrast, $\mathrm{LSCl}$ can be used to estimate blood perfusion in the capillary bed the exact location of the ulcer $(12,14)$.

LSCl is already successfully applied in the assessment of burns and burn-related wounds (16). In people with diabetes, it has been used to assess the microvascular endothelial function of the arm in a population with type 1 diabetes (17). A stress test (post-occlusion peak test) was performed to differentiate between people with diabetes and a control group. Furthermore, a conference paper has reported a pilot study testing LSCI on the feet of 5 patients (3 with diabetes) (18). However, no additional clinical baseline information was provided and only 4 diabetic foot ulcers were measured in the 3 patients. Both studies concluded that $\mathrm{LSCl}$ could be used for diagnostic screening or monitoring of the capillary blood flow on a regular basis during treatment $(17,18)$. However, neither of these studies investigated whether LSCI is a method that can be reliably used in people with diabetes. Its stability, reproducibility, reliability and validity have not yet been investigated.

Our aim is to investigate the stability and reproducibility of LSCI for the assessment of microcirculation in the diabetic foot and its relation to currently used non-invasive blood pressure measurements and stress tests.

\subsection{METHODS}

For this prospective, single centre, observational cohort study, 33 consecutive participants with diabetic foot ulcers were recruited from the 'diabetic foot outpatient clinic' in Ziekenhuisgroep (Hospital Group) Twente, location Almelo, the Netherlands. Sample size calculation was not applicable, as no baseline information for $\mathrm{LSCl}$ in people with diabetic foot ulcers was available. All participants had diabetes mellitus type I or II, were 18 years or older and were able to undergo non-invasive blood pressure measurements. Participants with an ulcer on both feet or with a foot infection (International Working Group on the Diabetic Foot grade 2-4 (19)) were excluded. The study protocol was approved by the medical research ethics committee Twente (NL52422.044.15), and was registered in the Dutch Trial Register (NTR5116). All participants gave written informed consent before participation in the study. 
All participants underwent non-invasive blood pressure measurements consisting of brachial pressure, ankle pressure, toe pressure (measured at the hallux, or, in case the hallux was amputated, at the second toe), and transcutaneous oxygen pressure $\left(\mathrm{T} c p O_{2}\right)$ measurements (Periflux 6000, Perimed AB, Stockholm, Sweden); anklebrachial index $(\mathrm{ABI})$ and toe-brachial index $(\mathrm{TBI})$ were calculated. Participants were classified as non-ischemic, ischemic or critical-ischemic based on the lowest measured blood pressure using cut-off points based on international guidelines $(2,20)$ : participants with an ankle pressure $<50 \mathrm{mmHg}, \mathrm{ABI}<0.5$, toe pressure $<30 \mathrm{mmHg}$, $\mathrm{TBI}<0.3$, or $\mathrm{TcpO}_{2}<25 \mathrm{mmHg}$ were classified as 'critical-ischemic'; participants with an ankle pressure $50-70 \mathrm{mmHg}, \mathrm{ABI}$ 0.5-0.9, toe pressure $30-50 \mathrm{mmHg}$, TBI 0.3-0.7 or $\mathrm{TcpO}_{2} 25-30 \mathrm{mmHg}$ were classified as 'ischemic'; non-ischemic participants had values above those cut-off points, i.e.: ankle pressure $>70 \mathrm{mmHg}, A B I>0.9$, toe pressure $>50 \mathrm{mmHg}, \mathrm{TBI}>0.7$, and $\mathrm{TcpO}_{2}>30 \mathrm{mmHg}(2,20)$.

Following blood pressure measurements, multiple LSCI scans were performed in each participant (PeriCam PSI; Perimed AB, Stockholm, Sweden). Before use the system was calibrated with its calibration box, measuring a zero-perfusion area and a colloidal suspension of polystyrene particles to set the LSCI values on $0 \pm 5$ perfusion units (PU) and $250 \pm 5 \mathrm{PU}$ respectively. Both the dorsal and plantar side of the foot were scanned, but only the ulcerated side was used in data analysis. The scans were made with approximately $25 \mathrm{~cm}$ distance between the system and the foot, a framerate of $10 \mathrm{fps}$, integration time of $6 \mathrm{~ms}$, a resolution of $1388 \times 1038$ pixels and a measurement area of $25 \times 15 \mathrm{~cm}$. The ulcerated foot was scanned twice by the principal investigator to calculate intra-observer variability. For 25 participants, a complete additional LSCI scan of the ulcer was performed by a trained health professional (registered physician assistant with $>10$ years of experience in treating diabetic foot disease) as second observer to calculate inter-observer variability.

Both the non-invasive blood pressure measurement and the $\mathrm{LSCl}$ scans were performed in a temperature controlled hospital room $\left(21 \pm 1^{\circ} \mathrm{C}\right)$. All patients underwent wound treatment before the perfusion measurements took place, during which procedure the foot was able to acclimatize to this room temperature. The $\mathrm{LSCl}$ scan methodology is schematically shown in Fig. 1. Baseline microcirculation was determined in the first period of the scan, and defined as 'no visual changes in the mean LSCl signal value for 30 seconds'. Subsequently, two stress tests (postocclusion peak and elevation test, as described below) were performed, in order to increase a potential difference between patient populations and to assess microcirculation and endothelial function in more detail. Between and after those stress tests an extra baseline measurement of 30 seconds was performed to monitor the stability of LSCl over time. 

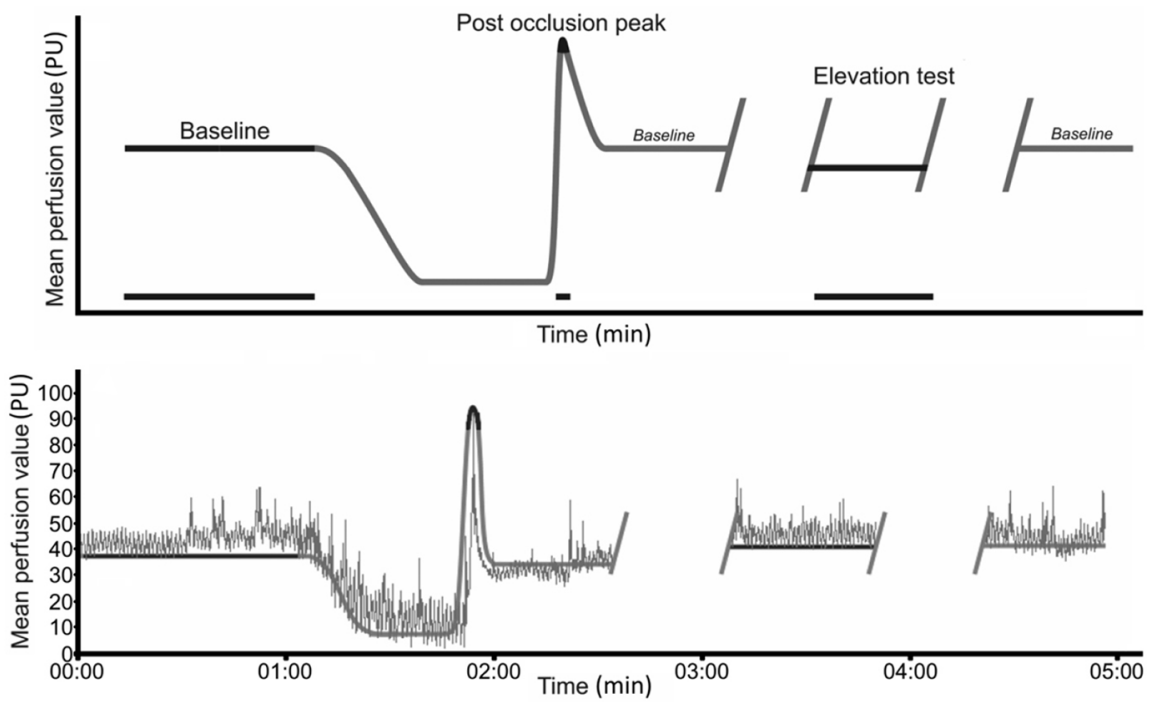

Figure 1 Top: Schematic overview of different stress tests and Laser Speckle Contrast Imaging signal over time with measurement periods shown in black. Baselines and elevation test all had a minimum duration of 30 seconds. Bottom: Example of the outcomes of the LSCl scan in one participant, with mean perfusion values for each ROI over time.

During the post-occlusion peak test, blood flow in the foot was interrupted with a pressure cuff ( $\max 260 \mathrm{mmHg}$ ) around the ankle. The pressure was released after 30 seconds. The maximum perfusion value during the post-occlusive reactive hyperaemia was measured as outcome. During the elevation test, the foot was lifted on a foam block of $30 \mathrm{~cm}$ height (with the scan paused during placement). Elevation test measurements consisted of a minimum of 30 seconds of a stable signal in the elevated foot, with its mean value as outcome.

The scans were quantified by measuring the perfusion of five specified Regions Of Interest (ROI), referring to a physical position on the image: ulcer (ulcer location); ulcer edge (ulcer edges and surrounding $1 \mathrm{~cm}$ ); $\mathrm{TcpO}_{2}$ (probe measurement area); hallux (total visible hallux, or second toe when hallux was amputated); foot (total visible foot) - see Fig. 2. All ROls were drawn on the scan by the principal investigator using LSCIsoftware (PIMSoft version 1.5; Perimed AB, Stockholm Sweden). Positions of the ROI were adjusted in each timeframe by the principal investigator to correct for motion effects. In order to measure the influence of the different assessors on the rating of the $\mathrm{LSCl}$ scans, for the same 25 participants that received an extra scan from the trained health professional an extra analysis was performed by an independent researcher who was not involved in the LSCI scans. This researcher quantified the scan from the trained health professional via the method described above. 

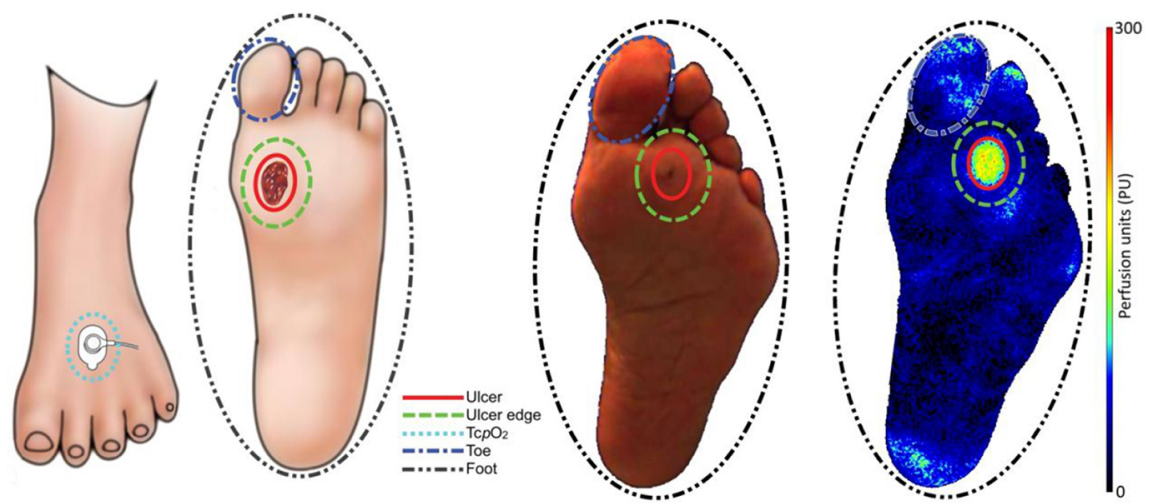

Figure 2 Left: Schematic overview of the different Regions of Interest for perfusion measurements; ulcer location (edge) varied between participants. Middle and right: outcomes of an LSCI scan in one participant, with colour image (middle) and perfusion image (right).

Zero perfusion units from the background were removed from the picture and not used in the data analysis, because we excluded all zero-value pixels during calculations. The stability over time of the baseline measurements was analysed with linear mixed models. Intra- and inter-observer correlations were calculated using intra-class correlations between and within different observers. Values $>0.7$ were considered high agreement (21). For the relation of LSCl and currently used non-invasive blood pressure measurements, Pearson's $r$ was calculated. A two-way random effect ANOVA was conducted to compare the inter-assessor reliability. One-way ANOVA was performed to calculate the difference in microcirculation between the three patient populations (non-ischemic, ischemic and critical-ischemic), followed by post-hoc Bonferroni corrections for multiple comparisons between groups. With 3 conditions and 5 ROls per patient, a total of 15 measurements were available for analyses. The relation between baseline measurement and post-occlusive peak or elevation test measurements was calculated with a Pearson's $r$. Alpha was set at $\leq 0.05$. Analyses were performed using SPSS version 21.0 (SPSS Inc. Chicago, IL, USA) and Microsoft Excel 2016 (Microsoft Corporation, Redmond, WA, USA). 


\subsection{RESULTS}

The majority of the 33 included participants were males, mean age was around $64.9 \pm 13.6$ years, and most were diagnosed with type 2 diabetes (Table 1).

Table 1: Participant characteristics $(\mathrm{N}=33)$

\begin{tabular}{|c|c|c|}
\hline Characteristics & $\mathrm{N}$ & $\%$ \\
\hline \multicolumn{3}{|l|}{ Gender } \\
\hline Male & 28 & 85 \\
\hline Female & 5 & 15 \\
\hline \multicolumn{3}{|l|}{ Age (years) } \\
\hline mean $\pm S D$ (range) & \multicolumn{2}{|c|}{$64.9 \pm 13.6(38-85)$} \\
\hline \multicolumn{3}{|l|}{ Diabetes Mellitus } \\
\hline Type 1 & 2 & 6 \\
\hline Type 2 & 31 & 94 \\
\hline \multicolumn{3}{|l|}{ Ulcer location } \\
\hline MTP1/dig 1 & 13 & 39 \\
\hline MTP2-3/dig 2-3 & 9 & 27 \\
\hline MTP4-5/dig 4-5 & 8 & 24 \\
\hline Other & 3 & 9 \\
\hline \multicolumn{3}{|l|}{ Ischemia } \\
\hline Non-ischemic & 11 & 33 \\
\hline Ischemic & 14 & 42 \\
\hline Critical-ischemic & 8 & 24 \\
\hline \multicolumn{3}{|l|}{ Neuropathic } \\
\hline Neuropathic impairment & 29 & 88 \\
\hline No neuropathic impairment & 4 & 12 \\
\hline
\end{tabular}

Note: SD = standard deviation; MTP = metatarsophalangeal joint; dig = digitum

We found no significant differences between the three baselines over time (before stress tests $46.05 \pm 13.15 \mathrm{PU}$, between stress tests $48.33 \pm 16.01 \mathrm{PU}$ and after stress tests 47.65 $\pm 14.64 \mathrm{PU} ; r=0.058)$. A high and significant intra-observer agreement was found for all 15 measurements ( $r=0.711-0.950 ; p \leq 0.001$; Table 2). 


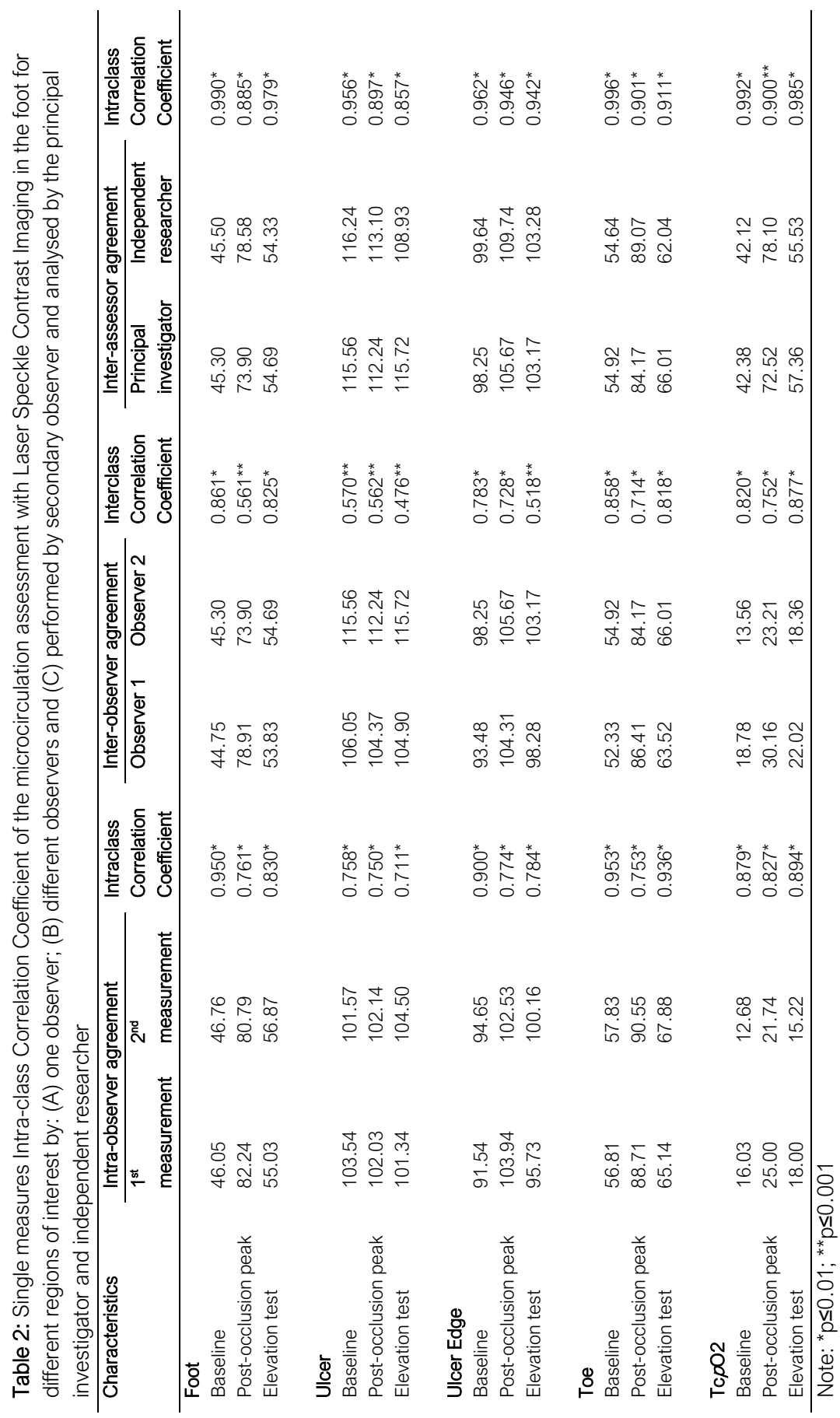


We found a high and significant inter-observer agreement for 10 out of 15 measurements (ICC=0.728-0.861; $p \leq 0.001)$, and a moderate and significant interobserver agreement for the remaining 5 measurements (ICC $=0.476-0.570 ; p \leq 0.005$; Table 2). The inter-assessor agreement was high and significant (ICC=0.857-0.996; $p \leq 0.001$; Table 2). Low correlations were found between $\mathrm{LSCl}$ and $\mathrm{ABI}$, toe pressure and $\mathrm{TcpO}_{2}$ (range -0.272-0.582; Table 3).

Table 3: Pearson's R correlations between Laser Speckle Contrast Imaging (LSCI) and noninvasive blood pressure measurements of the ulcerated foot for different Regions of Interest (ROI)

\begin{tabular}{|c|c|c|c|c|}
\hline ROI & Arm & Ankle & Toe & $\mathrm{Tc} p \mathrm{O}_{2}$ \\
\hline \multicolumn{5}{|l|}{ Foot } \\
\hline LSCI baseline & -0.272 & 0.112 & -0.048 & -0.066 \\
\hline Post-occlusion peak & -0.162 & 0.202 & $0.382^{*}$ & 0.110 \\
\hline Elevation test & -0.018 & $0.477^{*}$ & $0.488^{*}$ & 0.180 \\
\hline \multicolumn{5}{|l|}{ Ulcer } \\
\hline LSCI baseline & -0.154 & -0.166 & 0.078 & 0.036 \\
\hline Post-occlusion peak & -0.047 & 0.048 & 0.227 & 0.070 \\
\hline Elevation test & 0.018 & 0.228 & $0.429^{*}$ & 0.245 \\
\hline \multicolumn{5}{|l|}{ Ulcer edge } \\
\hline LSCI baseline & -0.228 & 0.062 & 0.127 & -0.007 \\
\hline Post-occlusion peak & -0.188 & 0.238 & $0.390^{*}$ & 0.245 \\
\hline Elevation test & -0.062 & $0.367^{*}$ & $0.533^{*}$ & 0.216 \\
\hline \multicolumn{5}{|l|}{ Toe } \\
\hline LSCI baseline & & & 0.233 & \\
\hline Post-occlusion peak & & & $0.500^{*}$ & \\
\hline Elevation test & & & $0.582^{*}$ & \\
\hline \multicolumn{5}{|l|}{$\mathrm{Tc} p \mathrm{O}_{2}$} \\
\hline LSCI baseline & & & & 0.062 \\
\hline Post-occlusion peak & & & & 0.232 \\
\hline Elevation test & & & & 0.242 \\
\hline
\end{tabular}

Note: * ${ }^{*} \leq 0.05$ (two-tailed)

No significant differences in perfusion between the critical ischemic, ischemic and nonischemic populations were found during the LSCI baseline measurements (Table 4; Fig. 3). 
Table 4: ANOVA measurement with post hoc Bonferroni analysis for all participants with and without (critical-) ischemia for foot, hallux, ulcer and ulcer edge region of interest.

\begin{tabular}{|c|c|c|c|c|c|c|}
\hline \multirow{2}{*}{ Foot } & & \multirow[t]{2}{*}{$\begin{array}{l}\text { LSCl } \\
\text { Perfusion } \\
\text { (mean } \pm \mathrm{SD})\end{array}$} & \multirow[t]{2}{*}{$\begin{array}{r}\text { values } \\
\text { units }\end{array}$} & \multirow[t]{2}{*}{$\begin{array}{l}\text { ANOVA } \\
p \text { value }\end{array}$} & \multicolumn{2}{|c|}{$\begin{array}{l}\text { Post hoc analysis } \\
p \text { value }\end{array}$} \\
\hline & & & & & & \\
\hline \multirow[t]{3}{*}{ LSCI baseline } & Non-ischemic & $48.1 \pm 14.8$ & & & $\mathrm{NI}-\mathrm{I}$ & 1.000 \\
\hline & Ischemic & $46.9 \pm 12.7$ & & 0.575 & $\mathrm{NI}-\mathrm{Cl}$ & 0.947 \\
\hline & Critical-ischemic & $41.8 \pm 12.2$ & & & $\mathrm{I}-\mathrm{Cl}$ & 1.000 \\
\hline \multirow[t]{3}{*}{ Post-occlusion peak } & Non-ischemic & $96.3 \pm 29.3$ & & & $\mathrm{NI}-\mathrm{I}$ & 0.284 \\
\hline & Ischemic & $79.6 \pm 18.4$ & & $0.044^{*}$ & $\mathrm{NI}-\mathrm{Cl}$ & $0.045^{*}$ \\
\hline & Critical-ischemic & $67.5 \pm 24.7$ & & & $\mathrm{I}-\mathrm{Cl}$ & 0.791 \\
\hline \multirow[t]{3}{*}{ Elevation test } & Non-ischemic & $63.9 \pm 16.2$ & & & $\mathrm{NI}-\mathrm{I}$ & 0.468 \\
\hline & Ischemic & $56.1 \pm 10.0$ & & $0.003^{* *}$ & $\mathrm{NI}-\mathrm{Cl}$ & $0.003^{* *}$ \\
\hline & Critical-ischemic & $41.0 \pm 14.1$ & & & $\mathrm{I}-\mathrm{Cl}$ & $0.046^{*}$ \\
\hline \multicolumn{7}{|l|}{ Toe } \\
\hline \multirow[t]{3}{*}{ LSCI baseline } & Non-ischemic & $68.0 \pm 28.9$ & & & $\mathrm{NI}-\mathrm{I}$ & 0.412 \\
\hline & Ischemic & $54.7 \pm 19.0$ & & 0.081 & $\mathrm{NI}-\mathrm{Cl}$ & 0.087 \\
\hline & Critical-ischemic & $45.0 \pm 11.8$ & & & $\mathrm{I}-\mathrm{Cl}$ & 0.953 \\
\hline \multirow[t]{3}{*}{ Post-occlusion peak } & Non-ischemic & $107.4 \pm 29.3$ & & & $\mathrm{NI}-\mathrm{I}$ & 0.167 \\
\hline & Ischemic & $86.7 \pm 20.2$ & & $0.007^{* *}$ & $\mathrm{NI}-\mathrm{Cl}$ & $0.006^{\star *}$ \\
\hline & Critical-ischemic & $66.7 \pm 29.6$ & & & $\mathrm{I}-\mathrm{Cl}$ & 0.268 \\
\hline \multirow[t]{3}{*}{ Elevation test } & Non-ischemic & $83.0 \pm 28.7$ & & & $\mathrm{NI}-\mathrm{I}$ & 0.196 \\
\hline & Ischemic & $65.6 \pm 21.6$ & & $0.001^{* * *}$ & $\mathrm{NI}-\mathrm{Cl}$ & $0.001^{* * *}$ \\
\hline & Critical-ischemic & $39.9 \pm 12.5$ & & & $\mathrm{I}-\mathrm{Cl}$ & $0.047^{*}$ \\
\hline \multicolumn{7}{|l|}{ Ulcer } \\
\hline \multirow[t]{3}{*}{ LSCI baseline } & Non-ischemic & $113.4 \pm 23.7$ & & & $\mathrm{NI}-\mathrm{I}$ & 0.490 \\
\hline & Ischemic & $96.3 \pm 28.9$ & & 0.370 & $\mathrm{NI}-\mathrm{Cl}$ & 1.000 \\
\hline & Critical-ischemic & $102.6 \pm 38.0$ & & & $\mathrm{I}-\mathrm{Cl}$ & 1.000 \\
\hline \multirow[t]{3}{*}{ Post-occlusion peak } & Non-ischemic & $114.4 \pm 18.6$ & & & $\mathrm{NI}-\mathrm{I}$ & 0.105 \\
\hline & Ischemic & $92.7 \pm 27.2$ & & 0.104 & $\mathrm{NI}-\mathrm{Cl}$ & 0.784 \\
\hline & Critical-ischemic & $101.4 \pm 26.2$ & & & $\mathrm{I}-\mathrm{Cl}$ & 1.000 \\
\hline \multirow[t]{3}{*}{ Elevation test } & Non-ischemic & $123.0 \pm 17.5$ & & & $\mathrm{NI}-\mathrm{I}$ & 0.053 \\
\hline & Ischemic & $96.1 \pm 27.1$ & & $0.005^{\star *}$ & $\mathrm{NI}-\mathrm{Cl}$ & $0.005^{\star *}$ \\
\hline & Critical-ischemic & $80.7 \pm 35.1$ & & & $\mathrm{I}-\mathrm{Cl}$ & 0.604 \\
\hline \multicolumn{7}{|l|}{ Ulcer edge } \\
\hline \multirow[t]{3}{*}{ LSCI baseline } & Non-ischemic & $102.8 \pm 27.5$ & & & $\mathrm{NI}-\mathrm{I}$ & 0.396 \\
\hline & Ischemic & $87.5 \pm 19.1$ & & 0.181 & $\mathrm{NI}-\mathrm{Cl}$ & 0.288 \\
\hline & Critical-ischemic & $83.2 \pm 28.7$ & & & $\mathrm{I}-\mathrm{Cl}$ & 1.000 \\
\hline \multirow[t]{3}{*}{ Post-occlusion peak } & Non-ischemic & $123.0 \pm 26.5$ & & & $\mathrm{NI}-\mathrm{I}$ & $0.016^{*}$ \\
\hline & Ischemic & $96.1 \pm 21.5$ & & $0.006^{* *}$ & $\mathrm{NI}-\mathrm{Cl}$ & $0.014^{*}$ \\
\hline & Critical-ischemic & $91.5 \pm 15.8$ & & & $\mathrm{I}-\mathrm{Cl}$ & 1.000 \\
\hline \multirow[t]{3}{*}{ Elevation test } & Non-ischemic & $116.4 \pm 21.6$ & & & $\mathrm{NI}-\mathrm{I}$ & $0.039^{*}$ \\
\hline & Ischemic & $93.8 \pm 19.6$ & & $\leq 0.001^{* * *}$ & $\mathrm{NI}-\mathrm{Cl}$ & $\leq 0.001^{* * *}$ \\
\hline & Critical-ischemic & $70.8 \pm 23.6$ & & & $\mathrm{I}-\mathrm{Cl}$ & 0.062 \\
\hline
\end{tabular}

Note: $\mathrm{NI}-\mathrm{I}$ = Non-ischemic compared to Ischemic, $\mathrm{NI}-\mathrm{Cl}$ = Non-ischemic compared to Criticalischemic, $\mathrm{I}-\mathrm{Cl}=$ Ischemic compared to Critical-ischemic;

${ }^{*} \mathrm{p} \leq 0.05,{ }^{* *} \mathrm{p} \leq 0.01,{ }^{* * *} \mathrm{p} \leq 0.001$ 

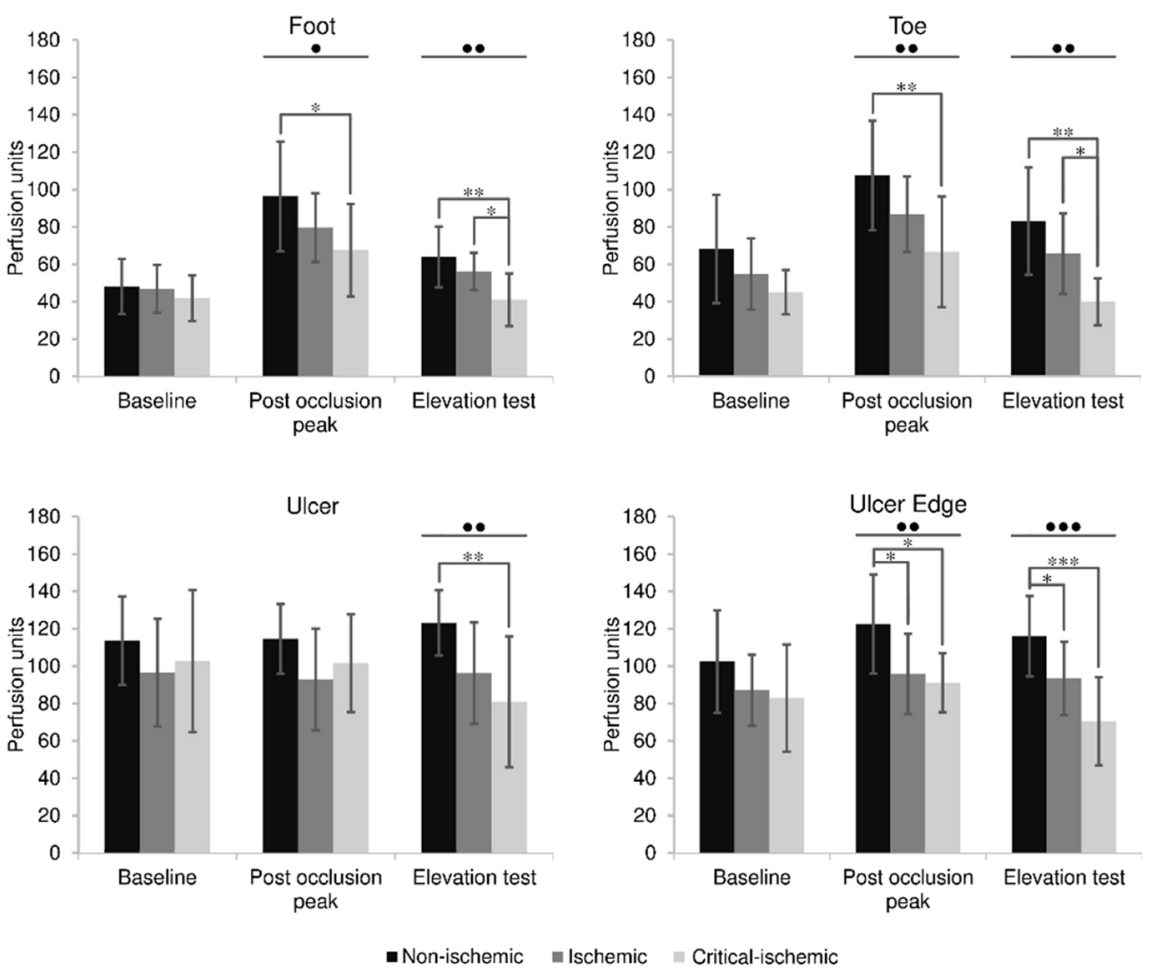

Figure 3: $\mathrm{LSCl}$ values (perfusion units, mean $\pm \mathrm{SD}$ ) of all participants with and without (critical) ischemia for foot, toe, ulcer and ulcer edge ROls.

Note: ANOVA differences between groups are marked with $\bullet(p \leq 0.05), \boldsymbol{\bullet}(p \leq 0.01), \boldsymbol{\cdots}(p \leq 0.001)$; post-hoc Bonferroni significant differences between groups are marked with * $(p \leq 0.05)$, ${ }^{* *}(p \leq 0.01),{ }^{* * *}(p \leq 0.001)$.

The perfusion during both stress tests was significantly higher for the non-ischemic population in comparison with the critical-ischemic population (Table 4; Fig. 3). The only exception was the post-occlusion peak for the ulcer ROI, which did not differ between populations. Further, perfusion was significantly higher in the ischemic vs. the critical-ischemic populations for the foot and toe ROls during the elevation test, and significantly higher for the non-ischemic vs. the ischemic populations in the ulcer edge ROI during both stress tests, (Table 4; Fig. 3).

Moderate to good correlations (range 0.630-0.839; $p \leq 0.001$ ) were found between the individual baseline measurements and the post-occlusive peak and elevating test values. Correlations for the post-occlusion peak were lower compared to elevation test values for all ROls (Foot: 0.649 vs 0.779 ; Toe: 0.680 vs 0.833 ; Ulcer: 0.792 vs 0.798 ; Ulcer edge: 0.630 vs 0.839 ). Correlations between baseline and stress test values for the different patient populations (non-ischemic, ischemic and critical-ischemic) show 
similar results (range 0.381-0.941), where post-occlusion peak were lower compared to elevation test values for all measurements except the toe values in the critical ischemic group (Fig. 4).

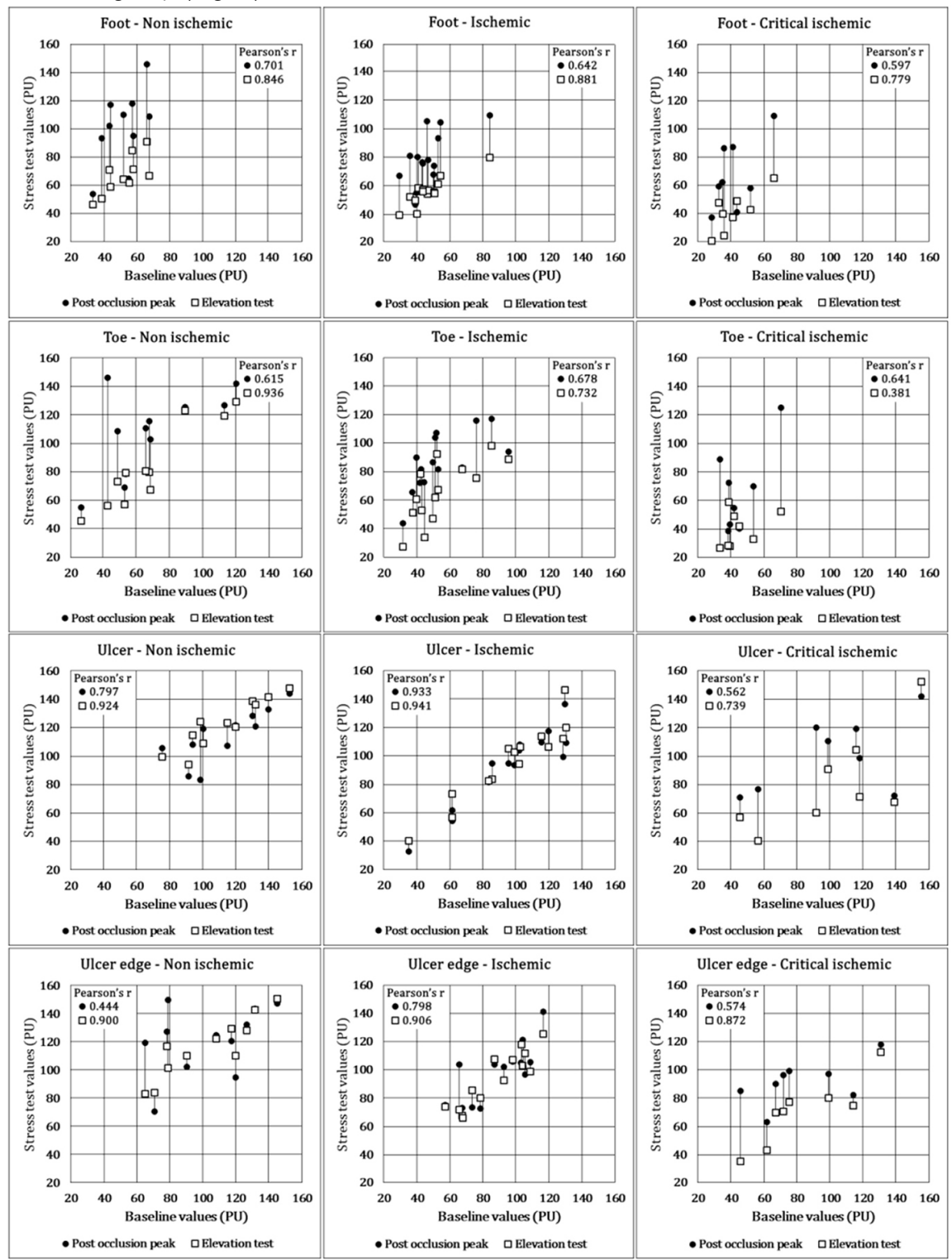

Figure 4: LSCI baseline values compared to stress test values (post occlusion peak and Elevation test) of all participants with and without (critical-)ischemia for the foot, toe, ulcer and ulcer edge ROls.

Note: Vertical lines connect corresponding measurements of the same patients. 


\subsection{DISCUSSION}

In this prospective cohort study, we investigated the reproducibility and stability of LSCI for the assessment of microcirculation in people with diabetic foot ulcers, and its relation with currently used non-invasive blood pressure measurements. We found high intra-observer agreements, moderate-to-high inter-observer agreements and high inter-assessor agreements; low correlations between LSCI and currently used noninvasive blood pressure measurements. LSCI was able to differentiate between nonischemic, ischemic and critical-ischemic patients, especially during elevated blanching. LSCI can be regarded as a suitable technique to assess microcirculation in people with diabetic foot ulcers: it is stable and reproducible, non-invasive and noncontact, it does not disturb the blood flow, nor causes it damage to the tissues, and it can easily be implemented in routine outpatient clinical practice $(17,18,22)$.

Our findings regarding the intra-observer agreement are in line with other studies on $\mathrm{LSCl}$ for microcirculation assessment in other clinical populations, such as patients with clinical signs of peripheral arterial disease (23) and healthy populations $(24,25)$. In the study measured on peripheral arterial disease, perfusion was measured using LSCI in 63 feet of patients (23). They investigated reproducibility of 8 measurements, and found no significant differences between the measurements. Furthermore, the reproducibility of a post-occlusive peak test was measured for LSCI when measured on different days on the forearm of 28 healthy volunteers, and was found to be high (25). Finally, in a study on 10 subjects similar results were found compared to our work concerning performance of the post-occlusive peak test with $\mathrm{LSCl}$, as they also found moderate-to-high inter-observer and intra-observer reproducibility of baseline, peak and plateaus (26).

This study was the first to also investigate inter-assessor agreement. We found high correlations, indicating that the influence of different assessors on the ROI placement or the removal of movement artefacts in the results is limited. This implies that data extraction from scans can be safely done by different technicians. However, it is still a useful avenue for future research to automate this step, as the current analyses are time-consuming and thereby a barrier for effective clinical implementation.

The low correlation between LSCl and conventional blood pressure measurements was anticipated, and in line with the aforementioned clinical population of 63 peripheral arterial disease patients (23). We confirm their conclusion that baseline perfusion values of $\mathrm{LSCl}$ are not correlated with non-invasive blood pressure measurements (e.g. $\mathrm{ABI}$ and tcPO2). This suggests that $\mathrm{LSCl}$ measurements differ from currently used noninvasive blood pressure measurements. This is likely the result of measuring at different area and depth with LSCI in comparison to non-invasive blood pressure measurement. 
For example, toe and ankle pressure (and related indices) or $\mathrm{TcpO}_{2}$ do not necessarily reflect the microcirculatory flow at the ulcer site, the ulcer edge or the whole foot. With $\mathrm{LSCl}$, the whole foot can be measured, as well as individual ROls. The penetration depth of LSCI is approximately $0.3 \mathrm{~mm}$ (26), measuring circulation predominantly in the skin arterioles, whereas non-invasive blood pressure is derived from arteries. Although non-invasive blood pressure measurements are the current standard for initial prediction of diabetic foot ulcer healing tendency (2), we expect that with the measurement of microcirculation in the arterioles closer to the ulcer, a better indication might be available of the situation at or around the ulcer. This might lead to an improvement of the moderate predictions of the healing tendency currently achieved (9).

A requirement to improve this healing tendency prediction, is that $\mathrm{LSCl}$ should discriminate between critical-ischemic, ischemic and non-ischemic patients. Although we found different perfusion values for each patient population, with non-ischemic patients having the highest perfusion values and critical-ischemic the lowest, the differences were not significant during the baseline measurements or at the ulcer ROI. Both stress tests enlarged these differences and reached statistical significance in most ROls. This indicates that potential usefulness of $\mathrm{LSCl}$ lies especially in combination with those stress tests. The increased perfusion value during the elevated blanching tests could be explained by the veno-arteriolar reflex causing vasoconstriction of the microvascular bed when the foot is lowered (27). It could be possible that the lifting of the leg causes a vasodilatation and increases the perfusion values and differences between the non-ischemic and critical-ischemic patient population during the stress test.

Differences between the three populations were smaller at the ulcer ROI than at the ulcer edge ROI. This could be explained by the angiogenesis and reepithelialisation that form from the ulcer edge towards the centre of the ulcer area, and with more microcirculatory activity in non-ischemic patients compared to the (critical-)ischemic patient population. The difference in LSCl at the ulcer location between non-ischemic and (critical-)ischemic patients was smaller. After debridement it is possible that fluid, nutrients, and immune-competent cells are transported to the ulcer and increase the LSCI signal (28), and all our participants were measured after debridement. Other studies have shown similar findings, for example when microcirculation in and around diabetic foot ulcers was measured with hyperspectral imaging $(29,30)$. They found a significant negative correlation between the hyperspectral imaging signal in intact skin adjacent to the edge of the ulcer and ulcer healing (30). We expect that LSCI may complement non-invasive blood pressure measurement, to assess the microcirculation in this population. Based on the current results, we suggest that microcirculation in the ulcer edge could be the best indicator, and application of different stress test during the measurements is needed. 
Different optical imaging techniques are also available to monitor microcirculation in diabetic foot ulcers. For example Laser Doppler Perfusion Imaging (LDPI), Hyperspectral Imaging (HSI) and Photoacoustic Imaging (31). Each technique has their own advantages and disadvantages and can be used to measure different characteristics of the microcirculation in tissue or the diabetic foot (31). The noncontact and full field imaging possibilities of LSCI, LDPI and HSI are great advantages for clinical measurements. Furthermore, the compact design, low cost and short imaging time of the LSCl make it an interesting technique to measure perfusion in the diabetic foot and to investigate the feasibility of LSCI in a clinical setting.

This study has limitations. First, the differentiation between the three patient populations was based on non-invasive blood pressure measurements. This is in line with international guidelines (2), but still includes the limitations of these non-invasive tests as discussed above. Unfortunately, no other non-invasive criteria are currently available. The use of invasive or extensive diagnostic techniques, like angiography or duplex, was not desirable in this study. We did not want to expose participants to invasive tests when these were not needed for their regular treatment. A potential effect of this limitation is dividing participants into the wrong category of ischemia, while this could not be checked with invasive diagnostics. This could result in a different distribution of the patient populations and may influence the results, as a different distribution of patients based on other diagnostic techniques would result in different mean $\mathrm{LSCl}$ values for each patient population.

Another limitation was the relatively small study size, and the not entirely equal distribution of the number of the non-ischemic, ischemic and critical-ischemic participants. However, our total of 33 participants was sufficient to obtain first insights in the use of $\mathrm{LSCl}$, and the number of participants is comparable to related studies $(23,30)$. With the variations seen between patients with diabetic foot ulcers, assessment of $\mathrm{LSCl}$ in larger populations is needed in future research (11). Furthermore, no drug related medical background of the patients was included in this study. Therefore it was not possible to monitor and measure the potential effects of any vasoactive drugs on the measured perfusion values. This could be a confounding factor in our results.

We did not measure the temperature of the foot during each measurement. However, the $\mathrm{LSCl}$ scans were performed in a temperature controlled hospital room $\left(21 \pm 1^{\circ} \mathrm{C}\right)$ end the foot had sufficient time to acclimatise to this room temperature, but we cannot correct for possible differences in skin temperate between individual patients.

Another limitation was the neuropathic status of the patients. We measured the neurological impairment of all patients based on loss of protective sensation and were 
not able to perform a statistical analysis due to the small patient population without neurological impairment. Furthermore, we think that a better scaling and measurement of the degree of neurological impairment could be useful to show a better correlation between measured perfusion and neuropathic status. For example, a quantitative scaling method could give more insight in the correlation between measured perfusion and neurological impairment instead of measuring only the presence or absence of neurological impairment as we did in the current study.

This study provides first insights in the potential of $\mathrm{LSCl}$ in the assessment of microcirculation in the diabetic foot. Future research should examine the added diagnostic value of LSCI in clinical practice. As the aim of this study was to investigate the feasibility of LSCI, we did not investigate clinical outcomes in the current study. For this, a larger cohort of patients is needed that is followed over time, to link microcirculation assessment with clinical outcomes. The ability of LSCl to show significant differences between different patient groups indicates that threshold baseline LSCl-values to predict wound healing might be derived from such future research and could be used to predict healing outcome and help with diagnoses and the choice in adequate treatment options.

\subsection{CONCLUSION}

LSCI is a stable and reproducible technique for the assessment of microcirculation in people with diabetic foot ulcers and its result using various stress tests show significant differences between non-ischemic, ischemic and critical-ischemic patient populations. LSCI provides a new assessment method for microcirculation in this population and may complement non-invasive blood pressure techniques. 


\section{REFERENCES}

1. Bakker K, Apelqvist J, Lipsky B, Van Netten J, International Working Group on the Diabetic foot. The 2015 IWGDF Guidance documents on prevention and management of foot problems in diabetes: development of an evidence-based global consensus. Diabetes Metab Res Rev. 2016;32:2-6.

2. Hinchliffe R, Brownrigg J, Apelqvist J, Boyko EJ, Fitridge R, Mills J, et al. IWGDF guidance on the diagnosis, prognosis and management of peripheral artery disease in patients with foot ulcers in diabetes. Diabetes Metab Res Rev. 2016;32:37-44.

3. Aldworth J, Bache N Al, Hegelund MH, Hirst SM, Linnenkamp U, Magliano D, et al. IDF Diabetes Atlas 7th edition. 2015. 144 p.

4. Armstrong DG, Boulton AJM, Bus SA. Diabetic Foot Ulcers and Their Recurrence. N Engl J Med. 2017;376(24):2367-75.

5. Alexiadou K, Doupis J. Management of diabetic foot ulcers. Diabetes Ther. 2012;3(1):1-15.

6. Kerr M, Rayman G, Jeffcoate WJ. Cost of diabetic foot disease to the National Health Service in England. Diabet Med. 2014 Dec;31(12):1498-504.

7. Prompers L, Huijberts M, Schaper N, Apelqvist J, Bakker K, Edmonds M, et al. Resource utilisation and costs associated with the treatment of diabetic foot ulcers. Prospective data from the Eurodiale Study. Diabetologia. 2008;51(10):1826-34.

8. Prompers L, Schaper N, Apelqvist J, Edmonds M, Jude E, Mauricio D, et al. Prediction of outcome in individuals with diabetic foot ulcers: Focus on the differences between individuals with and without peripheral arterial disease. The EURODIALE Study. Diabetologia. 2008;51(5):747-55.

9. Brownrigg JRWW, Hinchliffe RJ, Apelqvist J, Boyko EJ, Fitridge R, Mills JL, et al. Performance of prognostic markers in the prediction of wound healing or amputation among patients with foot ulcers in diabetes: A systematic review. Diabetes Metab Res Rev. 2016;32:128-35.

10. Sumpio BE, Forsythe RO, Ziegler KR, van Baal JG, Lepantalo MJAA, Hinchliffe RJ. Clinical implications of the angiosome model in peripheral vascular disease. $\mathrm{J}$ Vasc Surg. 2013;58(3):814-26.

11. Elgzyri T, Larsson J, Thörne J, Eriksson K-FF, Apelqvist J. Outcome of Ischemic Foot Ulcer in Diabetic Patients Who Had no Invasive Vascular Intervention. Eur J Vasc Endovasc Surg. 2013;46(1):110-7.

12. Draijer M, Hondebrink E, Van Leeuwen T, Steenbergen W. Review of laser speckle contrast techniques for visualizing tissue perfusion. Lasers Med Sci. 2009;24(4):63951.

13. Boas DA, Dunn AK. Laser speckle contrast imaging in biomedical optics. J Biomed Opt. 2010;15(1):011109.

14. Biffi CA, Tuissi A. Stato dell'arte sulle tecniche di produzione additiva per metalli. Metall Ital. 2017;109(1):5-10.

15. Li H, Liu Q, Lu H, Li Y, Zhang HF, Tong S. Directly measuring absolute flow speed by frequency-domain laser speckle imaging. Vol. 22, Optics express. 2010. 21079 p.

16. Ganapathy P, Tamminedi T, Qin Y, Nanney L, Cardwell N, Pollins A, et al. Dual-imaging system for burn depth diagnosis. Burns. 2014;40(1):67-81.

17. de M. Matheus AS, Clemente ELS, de Lourdes Guimarães Rodrigues M, Torres 
Valença DC, Gomes MB. Assessment of microvascular endothelial function in type 1 diabetes using laser speckle contrast imaging. J Diabetes Complications. 2017;31(4):753-7.

18. Jayanthy AK, Sujatha N, Reddy MR, Narayanamoorthy VB. Non invasive blood flow assessment in diabetic foot ulcer using laser speckle contrast imaging technique. In: Wax A, Backman V, editors. Arch Ophthalmol. International Society for Optics and Photonics; 2014. p. 89521D.

19. Lipsky BA, Aragón-Sánchez J, Diggle M, Embil J, Kono S, Lavery L, et al. IWGDF guidance on the diagnosis and management of foot infections in persons with diabetes. Diabetes Metab Res Rev. 2016;32:45-74.

20. Spångeus A, Wijkman M, Lindström T, Engvall JE, Östgren CJ, Spångéus A, et al. Toe brachial index in middle aged patients with diabetes mellitus type 2: Not just a peripheral issue. Diabetes Res Clin Pract. 2013;100(2):195-202.

21. Landis JR, Koch GG. The Measurement of Observer Agreement for Categorical Data. Biometrics. 1977;33(1):159.

22. Allen J, Howell K. Microvascular imaging: techniques and opportunities for clinical physiological measurements. Physiol Meas. 2014;35(7):R91-141.

23. Katsui S, Inoue Y, Igari K, Toyofuku T, Kudo T, Uetake H. Novel assessment tool based on laser speckle contrast imaging to diagnose severe ischemia in the lower limb for patients with peripheral arterial disease. Lasers Surg Med. 2017;49(7):645-51.

24. Humeau-Heurtier A, Abraham P, Durand S, Mahé G. Excellent inter- and intra-observer reproducibility of microvascular tests using laser speckle contrast imaging. Clin Hemorheol Microcirc. 2013;58(3):439-46.

25. Roustit M, Millet C, Blaise S, Dufournet B, Cracowski JL. Excellent reproducibility of laser speckle contrast imaging to assess skin microvascular reactivity. Microvasc Res. 2010;80(3):505-11.

26. O'Doherty J, McNamara P, Clancy NT, Enfield JG, Leahy MJ. Comparison of instruments for investigation of microcirculatory blood flow and red blood cell concentration. J Biomed Opt. 2009;14(3):034025.

27. Rayman G, Hassan A, Tooke JE. Blood flow in the skin of the foot related to posture in diabetes mellitus. Br Med J (Clin Res Ed). 1986 Jan 11;292(6513):87-90.

28. Gonzalez AC de O, Costa TF, Andrade Z de A, Medrado ARAP. Wound healing - A literature review. An Bras Dermatol. 2016;91(5):614-20.

29. Yudovsky D, Nouvong A, Pilon L. Hyperspectral imaging in diabetic foot wound care. J Diabetes Sci Technol. 2010 Sep;4(5):1099-113.

30. Jeffcoate WJ, Clark DJ, Savic N, Rodmell PI, Hinchliffe RJ, Musgrove A, et al. Use of $\mathrm{HSI}$ to measure oxygen saturation in the lower limb and its correlation with healing of foot ulcers in diabetes. Diabet Med. 2015;32(6):798-802.

31. Mennes OA, van Netten JJ, Slart RHJ., Steenbergen W. Novel Optical Techniques for Imaging Microcirculation in the Diabetic Foot. Curr Pharm Des. 2018;24(12):1304-16. 



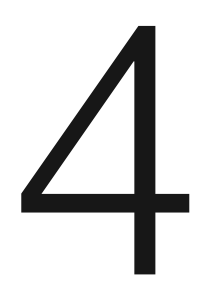

\section{The Association between Foot and Ulcer Microcirculation measured with Laser Speckle Contrast Imaging and Healing of Diabetic Foot Ulcers}




\section{ABSTRACT}

Background: Diagnosis of peripheral artery disease in people with diabetes and a foot ulcer using current non-invasive blood pressure measurements is challenging. Laser Speckle Contrast Imaging ( $\mathrm{LSCl}$ ) is a promising non-invasive technique to measure cutaneous microcirculation. This study investigated the association between microcirculation (measured with both $\mathrm{LSCl}$ and non-invasive blood pressure measurement) and healing of diabetic foot ulcers 12 and 26 weeks after measurement.

Methods: Sixty-one patients with a diabetic foot ulcer were included in this prospective, single-centre, observational cohort-study. LSCI scans of the foot, ulcer and ulcer edge were conducted, during baseline and post-occlusion hyperaemia. Non-invasive blood pressure measurement included arm, foot, and toe pressures and associated indices. Healing was defined as complete re-epithelialization and scored at 12 and 26 weeks.

Results: No significant difference between patients with healed or non-healed foot ulcers was found for both types of measurements $(p=0.135-0.989)$. ROC curves demonstrated moderate sensitivity (range 0.636-0.971) and specificity (range 0.4640.889), for LSCl and non-invasive blood pressure measurements.

Conclusions: No association between diabetic foot ulcer healing and LSCI-measured microcirculation or non-invasive blood pressure measurements was found. The healing tendency of diabetic foot ulcers is difficult to predict based on single measurements using current blood pressure measurements or LSCl. 


\subsection{INTRODUCTION}

Diabetes Mellitus is a metabolic disease and its patient population is growing worldwide, with a prevalence of $9.3 \%$ of the adults between 20 and 79 years old. A total of 463 million people are living with diabetes (1). One of the major complications of diabetes is diabetic foot disease. Mortality, high morbidity, costs and a reduced quality of life are all associated with diabetic foot disease (2-5). Peripheral neuropathy and peripheral artery disease (PAD) are both major causes for diabetic foot ulceration, and PAD also contributes to poor healing outcomes $(6,7)$. Recognizing the levels of ischemia of the lower limb arteries is therefore essential in the treatment of foot ulcers in people with diabetes. However, this has been identified by various researchers and clinicians as one of the key challenges in diabetic foot disease $(7,8)$.

Multiple methods are used to identify diabetic foot ulcers that are suspicious to poor healing as a result of PAD. Diagnostic arteriography and non-invasive blood pressure measurements are recommended in guidelines of the International Working Group on the Diabetic Foot (IWGDF) for the treatment of diabetic foot ulcers (9). Indications for vascular consultation include ankle blood pressure $<50 \mathrm{mmHg}$, toe pressure $<30 \mathrm{mmHg}$ and ankle / brachial index $(\mathrm{ABI})<0.5(6,10)$. However, these non-invasive blood pressure measurements have various disadvantages. For example, it has been shown that $A B I$ underestimates the prevalence of $P A D$ in people with diabetes due to the arterial circular calcification of the media and the consequent non-compressibility $(11,12)$. Toe pressure does not reflect the vascular situation at the ulcer location, and does not measure microcirculatory status. Microcirculation can be estimated with transcutaneous oxygen pressure measurements $\left(\mathrm{TcpO}_{2}\right)$, where a value above 25 $\mathrm{mmHg}$ has been demonstrated to predict ulcer healing potential; however, the presence of oedema can affect the accuracy of these measurements, and as this measurement is confined to the dorsal side of the foot it frequently does not reflect vascular status at the ulcer location $(6,10,11,13)$. In a recent systematic review on the performance of prognostic markers in the prediction of ulcer healing or amputation among foot ulcers in diabetes, it was concluded that wound healing was associated with a better perfused foot (skin perfusion pressure $\geq 40 \mathrm{mmHg}$, toe pressure $\geq 30$ $\mathrm{mmHg}$, or $\mathrm{TcpO}_{2} \geq 25 \mathrm{mmHg}$ ) (8). However, in most studies included in this systematic review, likelihood ratios of these tests in accurately predicting healing were small. While this is partly the result of co-existing factors (e.g. infection, comorbidities) also impacting on ulcer healing, it also suggests that other measurements overcoming the disadvantages of these non-invasive blood pressure measurements may result in better predictive values.

Novel optical imaging techniques such as Laser Speckle Contrast Imaging (LSCl) are available to complement the currently used non-invasive blood pressure 
measurements in people with diabetic foot disease (14). LSCI is a non-invasive optical imaging technique able to measure blood flow in the skin $(15,16)$. In general, the reproducibility of $\mathrm{LSCl}$ is high, and it has low inter-subject variability (17-20). LSCI is an interesting technique to measure blood flow in diabetic foot disease, because it has a widely validated track record of non-invasive in-vivo blood flow measurements compared to other established methods of large-area microcirculation (14). Furthermore, LSCl can provide non-invasive real-time feedback on changes in perfusion, and is able to monitor the microcirculation in the outer layer of the skin. Such microcirculation measurements give an indication of the perfusion directly in and around the ulcer, which overcomes the disadvantage of measurements such as $A B I$, toe pressure and $\mathrm{TcpO}_{2}$.

In a previous study among patients with a diabetic foot ulcer, we demonstrated that LSCl is a stable technique with a high inter- and intra-user reliability (21). We concluded that $\mathrm{LSCl}$ can be used in the clinical setting complementing non-invasive blood pressure measurements. However, for assessing its clinical benefit, insight in its prognostic accuracy in comparison to non-invasive blood pressure measurements is required. Therefore, the aim of this study is to investigate and compare the prognostic values of $\mathrm{LSCl}$ and non-invasive blood pressure measurements in relation to healing of diabetic foot ulcers.

\subsection{METHODS}

The clinical dataset used for this study was obtained as part of a larger study (21). This study was approved by a registered medical ethics committee and registered in the Dutch trial register (NTR5116). Thirty-three patients with a diabetic foot ulcer participated and both the non-invasive blood pressure measurements and LSCI measurements of each ulcerated foot was available. This dataset was supplemented with people with a diabetic foot ulcer who were eligible for regular treatment, as LSCl was implemented in daily practice following completion of the abovementioned study. All patients with a diabetic foot ulcer who were presenting at the outpatient clinic at ZGT Hospital, location Almelo, the Netherlands, were scanned with LSCl. If the patient fulfilled the inclusion criteria and gave permission to use the data for scientific purposes, the patient data was included in this study. All examinations used in the current study were part of regular treatment and therefore the second cohort of this study was exempt from medical ethical review according to the Medical Research Involving Human Subjects Act in the Netherlands. These two cohorts together form the participants of this current single centre, observational cohort study. All study actions were in line with the principles of the Declaration of Helsinki. 
Inclusion criteria were a confirmed diagnosis of type 1 or type 2 diabetes mellitus, and one foot ulcer (defined as break of the skin of the foot that involves at least the epidermis and part of the dermis (22)). Exclusion criteria were having multiple foot ulcers, an amputation of the forefoot or a more proximal location, moderate or severe foot infection (IWGDF grade 3 or 4; (23)), being incapacitated or undergoing cancer treatment. All patients were treated in accordance with the local protocol, which is based on the Dutch guidelines (24) and the IWGDF guidelines (25). Treatment consisted of offloading, ulcer debridement and wound dressings, antibiotic treatment in case of mild infection, and blood pressure measurements to assess PAD, and surgical revascularization when required. Regular blood pressure measurements included both the non-invasive blood pressure measurements (i.e. arm pressure, ankle pressure, $\mathrm{ABI}$, toe pressure and $\mathrm{TcpO}_{2}$ ). Regular microcirculatory measurements included $\mathrm{LSCl}$-scans in and around the ulcer location.

Measurements were performed after ulcer debridement, and consisted of first doing LSCI scans, followed by non-invasive blood pressure measurements. LSCI scans were performed of the ulcer foot with a PeriCam PSI NR (Perimed AB, Stockholm, Sweden). Either the plantar or the dorsal side of the foot was scanned, depending on the ulcer location, with the ulcer location to be included in the scan. Perfusion was expressed in Perfusion Units (PU). During the scan the patient lay supine on the examination table barefoot. After 5 minutes, for the patient to get used to the room temperature (kept between an ambient 21-22 degrees), the LSCI scans were acquired. During the scans three different time periods of interest (TOI) were measured: baseline, biological zero and post-occlusion hyperaemia measurements. The baseline was a measurement in the first stage of the scan when the measured perfusion was stable on visual inspection for 30 seconds. Subsequently, a cuff around the ankle was inflated to stop blood flow to the foot. During this time the perfusion dropped to the biological zero value of the patient. When the perfusion did not further decrease for 30 seconds the biological zero was measured. After this measurement the ankle cuff was released. The maximum measured blood flow after release of pressure was used as the post-occlusion hyperaemia value. During each TOI, different Regions of interest (ROI) of the foot were measured (i.e. foot, ulcer and ulcer edge). As described in detail in our previous paper (21), each ROI was manually selected in the scans in order to measure the mean perfusion of different areas of the foot and ulcer. The ROls were positioned at the beginning of each $\mathrm{TO}$ and repositioned during the scan to correct for possible movement of the foot during the scan (21). Non-invasive blood pressure measurements consisted of measuring arm pressure, ankle pressure, toe pressure and $\mathrm{T}_{\mathrm{C}} \mathrm{O}_{2}$, with a PeriFlux 6000 (Perimed AB, Stockholm, Sweden), all according to the manufacturer's instruction.

Each patient was classified as non-ischemic, ischemic or critical-ischemic based on non-invasive blood pressure measurements, following IWGDF criteria $(6,26)$. Patients 
were classified as critical-ischemic when $A B I \leq 0.39$, or ankle pressure $<50 \mathrm{mmHg}$, or toe pressure or $\mathrm{T}_{\mathrm{c}} \mathrm{O}_{2}<30 \mathrm{mmHg}$. Patients that are not classified as critical-ischemic but had an $\mathrm{ABI}$ between 0.4-0.79, or an ankle pressure between $50-100 \mathrm{mmHg}$, or a toe pressure or $\mathrm{TcpO}_{2}$ between $30-59 \mathrm{mmHg}$ were classified as ischemic. Patients were classified as non-ischemic with $\mathrm{ABI} \geq 0.8$ and an ankle pressure $>100 \mathrm{mmHg}$ and a toe pressure and $\mathrm{TcpO}_{2} \geq 60 \mathrm{mmHg}(6,10,27)$.

Clinical background and different parameters (Table 1) of the patient were obtained at baseline. The level of neuropathy was measured with a 10-grams Semmes-Weinstein monofilament (6), HbA1c was measured with blood tests and other parameters such as smoking were collected or measured during anamnesis. Follow-up for outcomes was until ulcer healing or for a maximum 26 weeks. Healing of the foot ulcer was defined as complete re-epithelialization of the ulcer without revascularization or major amputation (22) and was scored by an experienced clinician at 12 and 26 weeks. Patients who died, who underwent revascularization or major amputation were excluded.

\subsubsection{Statistical analysis}

To investigate differences between the healed and non-healed participants at 12 and 26 weeks, t-tests were conducted for all numerical variables and a $\mathrm{Chi}^{2}$ test for all categorical variables. Statistical relevance was considered with a p-value less than 0.05. ROC curves were created and the sensitivity and specificity of different parameters were calculated. The thresholds to calculate positive and negative likelihood ratio (LLR+ and LLR-) were chosen based on the highest combination of both sensitivity and specificity. A LLR- between $0.5-1$ or LLR+ between 1-5 indicates no to small change, while a LLR- between 0.1-0.5 or LLR+ between 5-10 were considered as moderate. LLR- below 0.1 or LLR+ above 10 were considered as large effect (8). 


\subsection{RESULTS}

A total of 61 patients were included. One patient died during follow-up, two patients underwent major amputation and five patients underwent revascularisation. Of the 53 patients included for analysis, 23 (43.4\%) healed within 12 weeks, 36 (67.9\%) in 26 weeks, while 17 patients (32.1\%) did not heal in 26 weeks or received revascularisation treatment (Figure 1, Table 1).

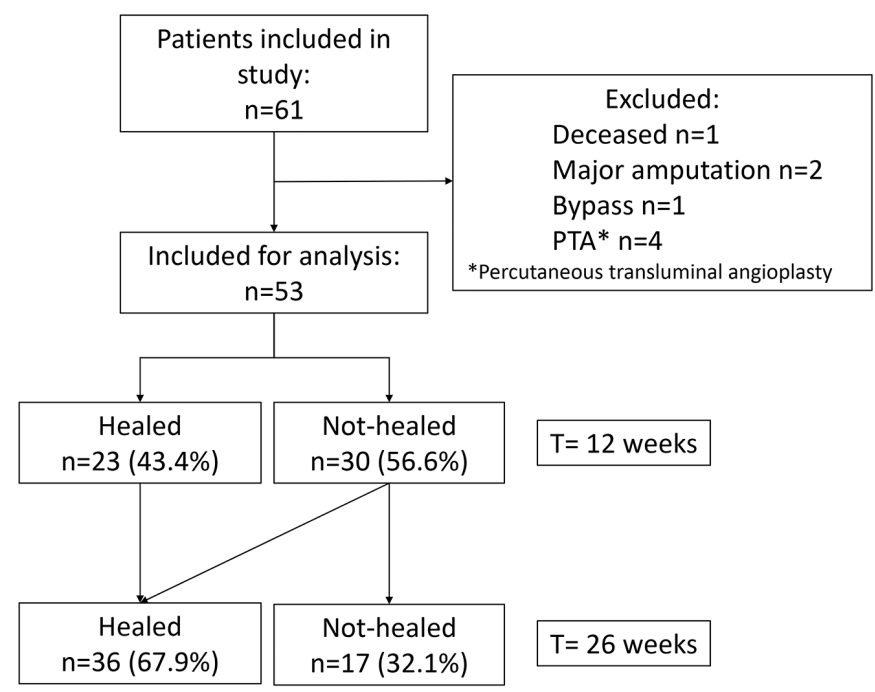

Figure 1: Schematic overview of patient population and clinical outcomes at 12 and 26 weeks.

Patients were on average 67 years, predominantly male, and with an average BMI of 29.7 (Table 1). Average healing percentages for the non-ischemic, ischemic and critical-ischemic groups were $4.3 \%, 69.6 \%$ and $26.1 \%$ at 12 weeks and $11.1 \%, 63.9 \%$ and $25.0 \%$ at 26 weeks. There were no significant differences between healed and non-healed patients at 12 weeks $(p=0.925)$ and also no significant differences between these groups at 26 weeks ( $p=0.275)$ (Table 1). Furthermore, for the majority of other patient characteristics, no significant difference was found between healers and nonhealers ( $p$-values ranging from 0.027-0.949; Table 1). 
Table 1: Patient characteristics at baseline, and separated between healed and non-healed patients at 12 and 26 weeks

\begin{tabular}{|c|c|c|c|c|c|c|c|c|}
\hline \multirow{2}{*}{\multicolumn{2}{|c|}{ Variable }} & \multirow{2}{*}{$\begin{array}{l}\text { Baseline } \\
\text { Mean } \pm \text { SD } \\
N(\%)\end{array}$} & \multicolumn{3}{|l|}{12 Weeks } & \multicolumn{3}{|l|}{26 weeks } \\
\hline & & & $\begin{array}{l}\text { Healed } \\
\text { Mean } \pm S D\end{array}$ & $\begin{array}{l}\text { Non-healed } \\
\text { Mean } \pm S D\end{array}$ & $\begin{array}{l}\mathrm{P} \text { - } \\
\text { value }\end{array}$ & $\begin{array}{l}\text { Healed } \\
\text { Mean } \pm S D\end{array}$ & $\begin{array}{l}\text { Non-healed } \\
\text { Mean } \pm S D\end{array}$ & $\begin{array}{l}P \text { - } \\
\text { value }\end{array}$ \\
\hline \multicolumn{2}{|c|}{ Patient characteristics } & $53(100 \%)$ & $23(43.4 \%)$ & $30(56.6 \%)$ & & $36(67.9 \%)$ & $17(32.1 \%)$ & \\
\hline \multicolumn{2}{|l|}{ Age (years) } & $66.7 \pm 12.8$ & $68.9 \pm 13.1$ & $65.1 \pm 12.7$ & 0.300 & $67.3 \pm 11.9$ & $65.7 \pm 15.1$ & 0.679 \\
\hline \multirow[t]{2}{*}{ Gender } & Male & $42(79.2 \%)$ & $18(78.3 \%)$ & $24(80 \%)$ & 0.877 & $30(83.3 \%)$ & $12(70.6 \%)$ & 0.286 \\
\hline & Female & $11(20.8 \%)$ & $5(21.7 \%)$ & $6(20 \%)$ & & $6(16.7 \%)$ & $5(29.4 \%)$ & \\
\hline \multicolumn{2}{|l|}{ Height $(\mathrm{cm})$} & $179.4 \pm 9.6$ & $179.1 \pm 11.1$ & $179.7 \pm 8.4$ & 0.858 & $179.7 \pm 10.7$ & $178.7 \pm 6.6$ & 0.744 \\
\hline \multicolumn{2}{|l|}{ Weight (Kg) } & $96.0 \pm 19.9$ & $98.0 \pm 21.4$ & $94.4 \pm 18.8$ & 0.546 & $96.9 \pm 20.1$ & $93.9 \pm 20.0$ & 0.660 \\
\hline \multicolumn{2}{|l|}{ BMI } & $29.7 \pm 5.5$ & $30.4 \pm 5.5$ & $29.2 \pm 5.6$ & 0.475 & $29.9 \pm 5.2$ & $29.4 \pm 6.4$ & 0.808 \\
\hline \multicolumn{2}{|c|}{$\mathrm{HbA} 1 \mathrm{c}(\mathrm{mmol} / \mathrm{mol})$} & $63.6 \pm 21.0$ & $59.5 \pm 14.4$ & $67.6 \pm 25.6$ & 0.221 & $63.0 \pm 15.9$ & $65.7 \pm 33.4$ & 0.729 \\
\hline \multirow[t]{4}{*}{ Smoking } & Yes & $11(52.4 \%)$ & $3(42.9 \%)$ & $8(57.1 \%)$ & 0.590 & $6(46.2 \%)$ & $5(62.5 \%)$ & 0.436 \\
\hline & No & $4(19.0 \%)$ & $1(14.3 \%)$ & $3(21.4 \%)$ & & $2(16.4 \%)$ & $2(25.0 \%)$ & \\
\hline & Stopped & $6(28.6 \%)$ & $3(42.9 \%)$ & $3(21.4 \%)$ & & $5(38.5 \%)$ & $1(12.5 \%)$ & \\
\hline & Unknown & 32 & 16 & 16 & & 23 & 9 & \\
\hline \multirow{2}{*}{$\begin{array}{l}\text { Diabetes } \\
\text { type }\end{array}$} & 1 & 3 (5.7\%) & $1(4.3 \%)$ & $2(6.7 \%)$ & 0.717 & $2(5.6 \%)$ & 1 (5.9\%) & 0.962 \\
\hline & 2 & 50 (94.3\%) & 22 (95.7\%) & $28(90.0 \%)$ & & 34 (94.4\%) & $16(88.2 \%)$ & \\
\hline \multirow{3}{*}{$\begin{array}{l}\text { Diabetes } \\
\text { duration }\end{array}$} & $\leq 10$ years & 20 (43.5\%) & $8(38.1 \%)$ & 12 (48.0\%) & 0.394 & $12(36.4 \%)$ & $8(61.5 \%)$ & 0.180 \\
\hline & $>10$ years & 26 (56.5\%) & 13 (61.9\%) & $13(52.0 \%)$ & & 21 (63.6\%) & $5(38.5 \%)$ & \\
\hline & Unknown & 7 & 2 & 5 & & 3 & 4 & \\
\hline \multirow[t]{3}{*}{ Dialysis } & Yes & $3(5.7 \%)$ & $1(4.3 \%)$ & 2 (6.7\%) & 0.266 & $2(5.6 \%)$ & 1 (5.9\%) & 0.998 \\
\hline & No & 47 (88.7\%) & 22 (95.7\%) & 25 (83.3\%) & & 32 (88.9\%) & 15 (88.2\%) & \\
\hline & In the past & 3 (5.7\%) & $0(0.0 \%)$ & $3(10.0 \%)$ & & $2(5.6 \%)$ & $1(5.9 \%)$ & \\
\hline \multirow[t]{2}{*}{ Infections } & Yes & $8(15.1 \%)$ & $4(17.4 \%)$ & $4(13.3 \%)$ & 0.683 & $5(13.9 \%)$ & $3(17.6 \%)$ & 0.721 \\
\hline & No & 45 (84.9\%) & 19 (82.6\%) & 26 (86.7\%) & & 31 (86.1\%) & $14(82.4 \%)$ & \\
\hline \multirow[t]{3}{*}{ Neuropathy } & y Yes & 48 (96.0\%) & $22(100.0 \%)$ & 26 (92.9\%) & 0.201 & 35 (100.0\%) & $13(86.7 \%)$ & 0.027 \\
\hline & No & $2(4.0 \%)$ & $0(0.0 \%)$ & $2(7.1 \%)$ & & $0(0.0 \%)$ & $2(13.3 \%)$ & \\
\hline & Unknown & 3 & 1 & 2 & & 1 & 2 & \\
\hline \multicolumn{2}{|c|}{ UT-classification } & & & & 0.776 & & & 0.704 \\
\hline & $O A$ & $4(7.5 \%)$ & $2(8.7 \%)$ & 2 (6.7\%) & & $3(8.3 \%)$ & 1 (5.9\%) & \\
\hline & $1 A$ & $30(56.6 \%)$ & $15(65.2 \%)$ & $15(50.0 \%)$ & & 22 (62.9\%) & $8(47.1 \%)$ & \\
\hline & $1 B$ & $1(1.9 \%)$ & $0(0.0 \%)$ & 1 (3.3\%) & & $0(0.0 \%)$ & 1 (5.9\%) & \\
\hline & $1 C$ & $1(1.9 \%)$ & $0(0.0 \%)$ & 1 (3.3\%) & & $1(2.8 \%)$ & $0(0.0 \%)$ & \\
\hline & $2 A$ & 5 (9.4\%) & 1 (4.3\%) & $4(13.3 \%)$ & & $3(8.3 \%)$ & $2(11.8 \%)$ & \\
\hline & $2 B$ & 5 (9.4\%) & $2(8.7 \%)$ & $3(10.0 \%)$ & & $3(8.3 \%)$ & $2(11.8 \%)$ & \\
\hline & $3 A$ & $3(5.7 \%)$ & 1 (4.3\%) & 2 (6.7\%) & & $2(5.6 \%)$ & 1 (5.9\%) & \\
\hline & $3 B$ & $3(5.7 \%)$ & $2(8.7 \%)$ & $1(3.3 \%)$ & & $2(5.6 \%)$ & 1 (5.9\%) & \\
\hline & $3 C$ & $1(1.9 \%)$ & $0(0.0 \%)$ & $1(3.3 \%)$ & & $0(0.0 \%)$ & 1 (5.9\%) & \\
\hline \multirow{2}{*}{$\begin{array}{l}\text { History of } \\
\text { ulcers }\end{array}$} & Yes & 31 (58.5\%) & 15 (65.2\%) & 16 (53.3\%) & 0.384 & 20 (55.6\%) & $11(64.7 \%)$ & 0.528 \\
\hline & No & 22 (41.5\%) & $8(34.8 \%)$ & $14(46.7 \%)$ & & $16(44.4 \%)$ & $6(35.3 \%)$ & \\
\hline \multicolumn{9}{|c|}{ Minor amputation } \\
\hline & Yes & $8(15.1 \%)$ & $2(8.7 \%)$ & $6(20.0 \%)$ & 0.255 & $5(13.9 \%)$ & $3(17.6 \%)$ & 0.721 \\
\hline & No & 45 (84.9\%) & $21(91.3 \%)$ & $24(80.0 \%)$ & & 31 (86.1\%) & $14(82.4 \%)$ & \\
\hline \multirow[t]{3}{*}{$\begin{array}{l}\text { Vascular } \\
\text { status }\end{array}$} & $\begin{array}{l}\text { Non- } \\
\text { ischemic }\end{array}$ & 7 (13.2\%) & $1(4.3 \%)$ & $6(20.0 \%)$ & 0.925 & $4(11.1 \%)$ & $3(17.6 \%)$ & 0.275 \\
\hline & Ischemic & $28(52.8 \%)$ & $16(69.6 \%)$ & 12 (40.0\%) & & 23 (63.9\%) & $5(29.4 \%)$ & \\
\hline & $\begin{array}{l}\text { Critical- } \\
\text { ischemic }\end{array}$ & $18(34.0 \%)$ & $6(26.1 \%)$ & 12 (40.0\%) & & $9(25.0 \%)$ & $9(52.9 \%)$ & \\
\hline
\end{tabular}

${ }^{*} \mathrm{p}<0.05$; note: UT-classification is the University of Texas Diabetic Wound Classification(28) 
There were no significant differences in any of the perfusion measurements between the healed and non-healed group, neither for LSCl at the foot, ulcer or ulcer edge, nor for any of the non-invasive blood pressure measurements ( $p$-values ranging from 0.136-0.983; Table 2).

Table 2: Mean values of Laser Speckle Contrast Imaging (in Perfusion Units (PU)) and noninvasive blood pressure measurements $(\mathrm{mmHg})$ at baseline and between patients with healed versus non-healed foot ulcers

\begin{tabular}{|c|c|c|c|c|c|c|c|}
\hline \multirow[t]{2}{*}{ Variable } & \multirow{2}{*}{$\begin{array}{l}\text { Baseline } \\
\text { Mean } \pm \text { SD } \\
N(\%)\end{array}$} & \multicolumn{3}{|l|}{12 Weeks } & \multicolumn{3}{|l|}{26 weeks } \\
\hline & & $\begin{array}{l}\text { Healed } \\
\text { Mean } \pm S D\end{array}$ & $\begin{array}{l}\text { Non-healed } \\
\text { Mean } \pm S D\end{array}$ & $\begin{array}{l}\mathrm{P}- \\
\text { value }\end{array}$ & $\begin{array}{l}\text { Healed } \\
\text { Mean } \pm S D\end{array}$ & $\begin{array}{l}\text { Non-healed } \\
\text { Mean } \pm S D\end{array}$ & $\begin{array}{l}\mathrm{P} \text { - } \\
\text { value }\end{array}$ \\
\hline \multicolumn{8}{|c|}{ Laser Speckle Contrast Imaging (PU) } \\
\hline \multicolumn{8}{|l|}{ Foot } \\
\hline Baseline & $50.3 \pm 14.6$ & $49.3 \pm 15.1$ & $51.1 \pm 14.5$ & .654 & $49.4 \pm 13.9$ & $52.3 \pm 16.3$ & .508 \\
\hline Biological zero & $12.8 \pm 7.7$ & $12.7 \pm 7.3$ & $12.8 \pm 8.1$ & .959 & $12.5 \pm 6.5$ & $13.5 \pm 10$ & .637 \\
\hline Post occlusion peak & $77.3 \pm 26.6$ & $76.7 \pm 24.4$ & $77.8 \pm 28.6$ & .889 & $77.4 \pm 23.2$ & $77.2 \pm 33.6$ & .983 \\
\hline \multicolumn{8}{|l|}{ Ulcer } \\
\hline Baseline & $104.8 \pm 34.6$ & $108.8 \pm 33$ & $101.8 \pm 36.1$ & .467 & $109.1 \pm 35.7$ & $95.8 \pm 31.2$ & .197 \\
\hline Biological zero & $25.2 \pm 15.3$ & $26.4 \pm 17.9$ & $24.3 \pm 13.2$ & .631 & $25 \pm 16.3$ & $25.7 \pm 13.4$ & .884 \\
\hline Post occlusion peak & $104.0 \pm 33.4$ & $107.8 \pm 32.6$ & $101.1 \pm 34.3$ & .473 & $108.2 \pm 35.2$ & $95.2 \pm 28.2$ & .190 \\
\hline \multicolumn{8}{|l|}{ Ulcer edge } \\
\hline Baseline & $92.2 \pm 30.7$ & $96.3 \pm 33.4$ & $89.1 \pm 28.6$ & .402 & $94.2 \pm 33.8$ & $88.1 \pm 23$ & .509 \\
\hline Biological zero & $20.1 \pm 10.7$ & $20.5 \pm 10.8$ & $19.8 \pm 10.9$ & .840 & $19.4 \pm 10$ & $21.7 \pm 12.3$ & .465 \\
\hline Post occlusion peak & $102.0 \pm 32.9$ & $108.1 \pm 33.9$ & $97.3 \pm 31.9$ & .239 & $104.8 \pm 35.3$ & $96 \pm 27.4$ & .373 \\
\hline \multicolumn{8}{|c|}{ Non-invasive blood pressure measurements $(\mathrm{mmHg})$} \\
\hline Ankle & $121.9 \pm 41.0$ & $121.3 \pm 46.3$ & $122.3 \pm 37.2$ & .931 & $126.9 \pm 41.4$ & $110.4 \pm 39$ & .183 \\
\hline Toe & $88.7 \pm 45.3$ & $97.7 \pm 45.1$ & $81.8 \pm 45.1$ & .220 & $95.4 \pm 45.2$ & $75.2 \pm 43.8$ & .136 \\
\hline $\mathrm{ABI}$ & $0.90 \pm 0.31$ & $0.88 \pm 0.3$ & $0.92 \pm 0.32$ & .698 & $0.94 \pm 0.32$ & $0.82 \pm 0.3$ & .188 \\
\hline TBI & $0.68 \pm 0.37$ & $0.73 \pm 0.32$ & $0.64 \pm 0.41$ & .410 & $0.73 \pm 0.38$ & $0.57 \pm 0.34$ & .151 \\
\hline $\mathrm{TcpO}_{2}$ & $47.9 \pm 17.5$ & $44.8 \pm 15.1$ & $50.3 \pm 19.1$ & .262 & $46.8 \pm 14.1$ & $50.1 \pm 23.6$ & .526 \\
\hline
\end{tabular}

When we compare the 95\% confidence intervals of both the $\mathrm{LSCl}$ perfusion measurements and non-invasive blood pressure measurements we see some nonsignificant differences (Figure 2). The LSCI perfusion values of the foot were lower for the healed group compared to the non-healed group for all TOl's and at both 12 and 26 weeks. While the LSCI perfusion at the ulcer and ulcer edge location were generally higher for those TOl's at 12 and 26 weeks.

The non-invasive blood pressure measurements of the healed group at 26 were generally higher than the non-healed group. At 12 weeks, only the toe-pressure and TBI were higher for the healed group compared to the non-healed group (Figure 2).

The ROC curves for LSCl scans and non-invasive blood pressure measurements demonstrated poor to moderate sensitivity and specificity (Figure 3). 


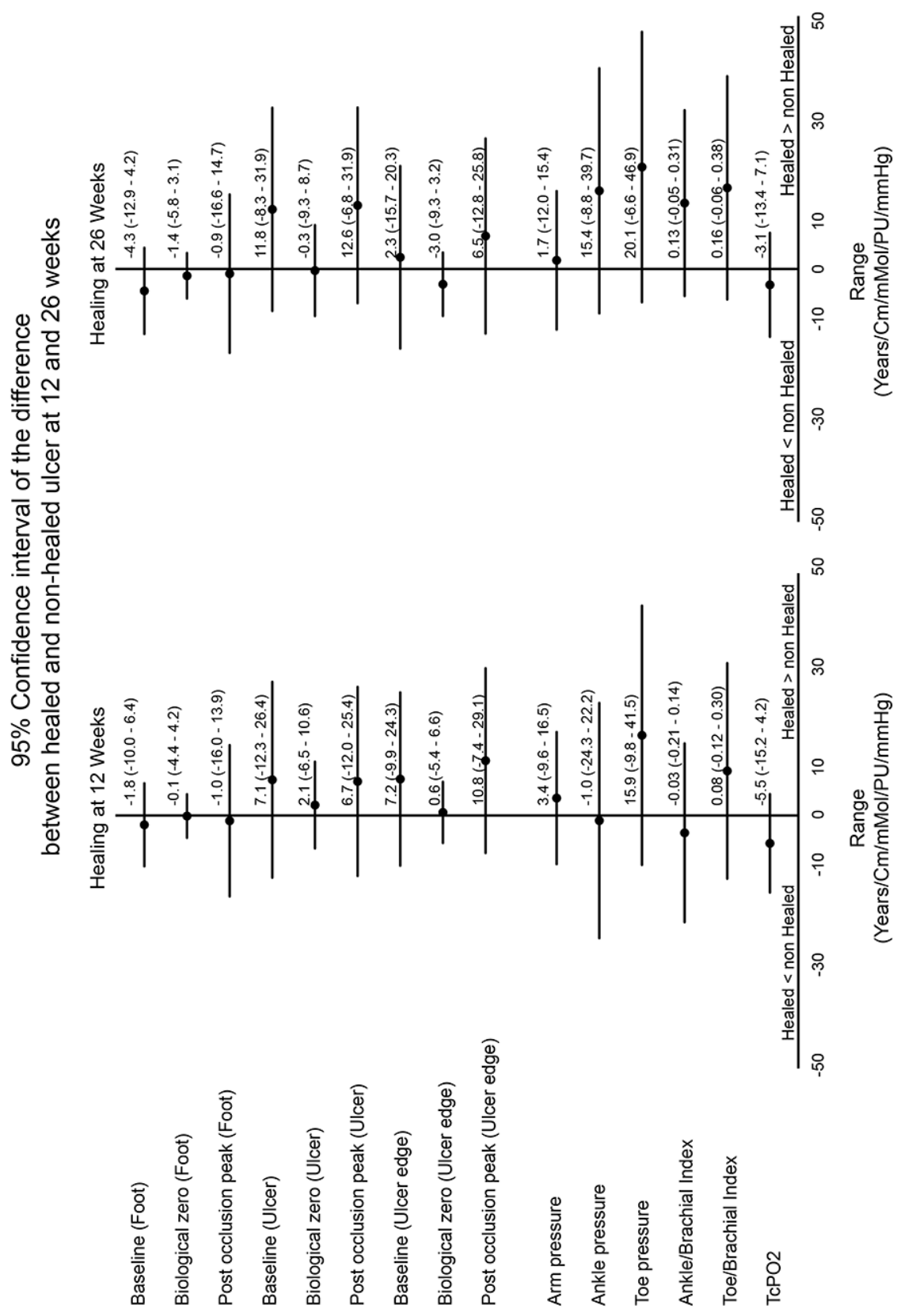

Figure 2: Difference in range of parameters between healed and non-healed patients at 12 and 26 weeks. Note: $\mathrm{ABI}$ and TBI interval are multiplied with 100 for better visualization. 

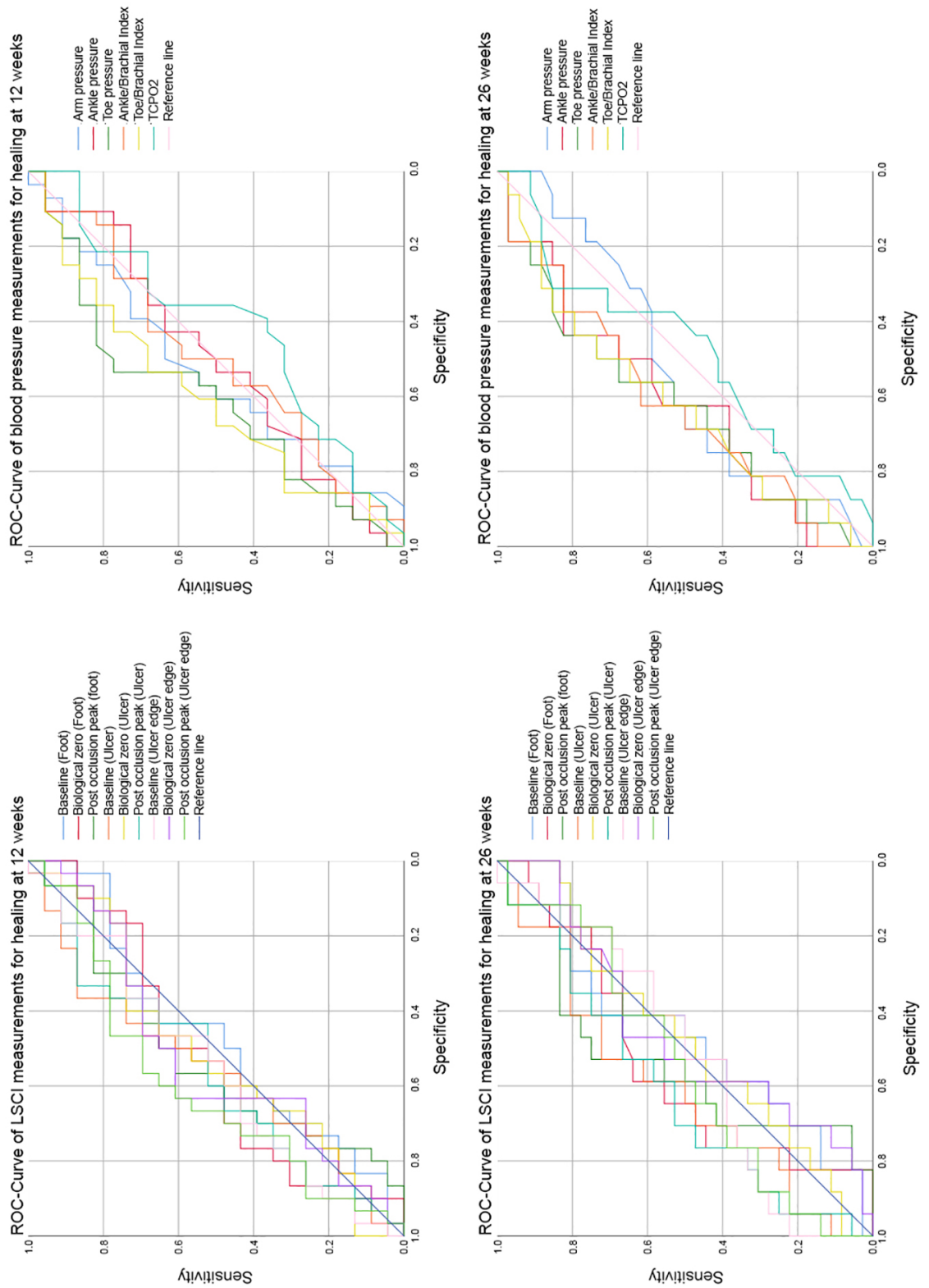

Figure 3: ROC curves showing sensitivity and specificity for Laser Speckle Contrast Imaging measurements and non-invasive blood pressure measurements as prognostic tests for ulcer healing at 12 or 26 weeks. 
Both LLR+ and LLR- showed a small to no effect (LLR+ 1.06-4.72; LLR- 0.36-0.89; Figure 3, Table 3). The largest effect for prognosis of healing at 12 weeks was found for LSCl at the ulcer during baseline or post-occlusive peak (LLR-: 0.36). The largest effect for prognosis of healing at 26 weeks was found for $\mathrm{LSCl}$ at the ulcer edge during baseline or post-occlusive peak (LLR+: 4.72 and 2.60) and for ankle and toe pressure (LLR-: 0.40).

Table 3: Threshold, sensitivity and specificity for non-invasive blood pressure measurements and Laser Speckle Contrast Imaging measurements, for healing after 12 weeks and 26 weeks

\begin{tabular}{|c|c|c|c|c|c|c|}
\hline 12 weeks & Threshold & AUC & Sensitivity & Specificity & LLR+ & LLR- \\
\hline \multicolumn{7}{|l|}{ Foot } \\
\hline Baseline & 43.5 PU & 0.467 & 0.696 & 0.400 & 1.16 & 0.76 \\
\hline Biological zero & 14.3 PU & 0.528 & 0.435 & 0.767 & 1.86 & 0.74 \\
\hline Post occlusion peak & $73.5 \mathrm{PU}$ & 0.517 & 0.609 & 0.567 & 1.40 & 0.69 \\
\hline \multicolumn{7}{|l|}{ Ulcer } \\
\hline Baseline & 84.3 PU & 0.558 & 0.870 & 0.367 & 1.37 & $0.36^{* *}$ \\
\hline Biological zero & 15.7 PU & 0.510 & 0.739 & 0.400 & 1.23 & 0.65 \\
\hline Post occlusion peak & 89.4 PU & 0.561 & 0.870 & 0.333 & 1.30 & $0.39^{* *}$ \\
\hline \multicolumn{7}{|l|}{ Ulcer edge } \\
\hline Baseline & 103.0 PU & 0.552 & 0.435 & 0.700 & 1.45 & 0.81 \\
\hline Biological zero & 19.9 PU & 0.519 & 0.609 & 0.633 & 1.66 & 0.62 \\
\hline Post occlusion peak & 96.6 PU & 0.603 & 0.696 & 0.567 & 1.61 & 0.54 \\
\hline Arm pressure & $130.5 \mathrm{mmHg}$ & 0.528 & 0.636 & 0.500 & 1.27 & 0.73 \\
\hline Ankle pressure & $153.0 \mathrm{mmHg}$ & 0.500 & 0.273 & 0.821 & 1.53 & 0.89 \\
\hline Toe pressure & $77.5 \mathrm{mmHg}$ & 0.608 & 0.773 & 0.536 & 1.66 & $0.42^{* *}$ \\
\hline Ankle Brachial Index & 0.83 & 0.494 & 0.682 & 0.429 & 1.19 & 0.74 \\
\hline Toe Brachial Index & 0.57 & 0.599 & 0.682 & 0.536 & 1.47 & 0.59 \\
\hline $\mathrm{Tc} p \mathrm{O}_{2}$ & $30.5 \mathrm{mmHg}$ & 0.416 & 0.818 & 0.214 & 1.04 & 0.85 \\
\hline 26 weeks & Threshold & & Sensitivity & Specificity & LLR+ & LLR- \\
\hline \multicolumn{7}{|l|}{ Foot } \\
\hline Baseline & 41.9 PU & 0.454 & 0.722 & 0.412 & 1.23 & 0.67 \\
\hline Biological zero & 10.9 PU & 0.540 & 0.639 & 0.588 & 1.55 & 0.61 \\
\hline Post occlusion peak & 62.3 PU & 0.541 & 0.750 & 0.529 & 1.59 & $0.47^{*}$ \\
\hline \multicolumn{7}{|l|}{ Ulcer } \\
\hline Baseline & 92.3 PU & 0.606 & 0.722 & 0.529 & 1.53 & 0.52 \\
\hline Biological zero & 12.7 PU & 0.469 & 0.750 & 0.294 & 1.06 & 0.85 \\
\hline Post occlusion peak & 109.5 PU & 0.609 & 0.472 & 0.765 & $2.01^{*}$ & 0.69 \\
\hline \multicolumn{7}{|l|}{ Ulcer edge } \\
\hline Baseline & 118.5 PU & 0.525 & 0.278 & 0.941 & $4.72^{*}$ & 0.77 \\
\hline Biological zero & 14.0 PU & 0.455 & 0.667 & 0.471 & 1.26 & 0.71 \\
\hline Post occlusion peak & 123.0 PU & 0.547 & 0.306 & 0.882 & $2.60^{*}$ & 0.79 \\
\hline Ankle pressure & $96.0 \mathrm{mmHg}$ & 0.619 & 0.824 & 0.438 & 1.46 & $0.40^{*}$ \\
\hline Toe pressure & $54.0 \mathrm{mmHg}$ & 0.626 & 0.824 & 0.438 & 1.46 & $0.40^{*}$ \\
\hline Ankle Brachial Index & 0.89 & 0.619 & 0.618 & 0.625 & 1.65 & 0.61 \\
\hline Toe Brachial Index & 0.51 & 0.618 & 0.735 & 0.500 & 1.47 & 0.53 \\
\hline $\mathrm{Tc} p \mathrm{O}_{2}$ & $30.5 \mathrm{mmHg}$ & 0.484 & 0.853 & 0.313 & 1.24 & $0.47^{*}$ \\
\hline
\end{tabular}

Note: AUC = Area Under the Curve; LLR+ = positive likelihood ratio; LLR- = negative likelihood ratio; $\mathrm{TcpO}_{2}=$ transcutaneous oxygen pressure measurements; *Small effect 
With no significant differences found between patients who healed and those who did not heal, we repeated all tests for the group of participants classified as ischemic only $(n=28)$. We chose to do so, because advanced blood pressure assessment is most important in this group from a clinical perspective, as these are patients for whom diagnosis and prognosis are in a grey area. However, these post-hoc analyses did not result in different findings (results not shown), again no differences were seen in blood pressure measurements between patients who healed and patients who did not heal.

\subsection{DISCUSSION}

The aim of this study was to investigate the association between foot and ulcer (micro)circulation (measured with both $\mathrm{LSCl}$ and non-invasive blood pressure measurement) and healing of diabetic foot ulcers at 12 and 26 weeks. We found no significant differences in any of the measurements between the group of healed and non-healed patients, neither at 12 nor 26 weeks. Positive and negative likelihood ratios showed no or only small effects. In our cohort, both LSCI and non-invasive blood pressure measurements were not useful as a standalone prognostic test for diabetic foot ulcer healing. This result is not in line with the outcomes of a recent systematic review by Forsythe et al. (8) in which it was concluded that some non-invasive blood pressure measurements may have prognostic value. However, the majority of studies included in this review showed similar likelihood ratios as found in the current study (8). This implies that prognostic quality of non-invasive blood pressure measurements on its own are not always a valuable predictor for healing of diabetic foot ulcers.

While our study had a different approach in calculating the cut-off values for different non-invasive bedside blood pressure measurement tests, we can still use the results of the studies included in Forsythe et al. (9) to put the effect of the found likelihood ratios in perspective. First, in our study, the cut-off values for the different blood pressure measurements were based on the optimal combination of both sensitivity and specificity for ulcer healing. This is a different approach compared to other studies in which they used fixed cut-off values and in which they calculated corresponding likelihood ratios based on those values. We chose this approach, because no cut-off values for $\mathrm{LSCl}$ measurements available yet. Therefore, it was necessary to find the cut-off values with the highest prognostic power. To compare LSCI with the noninvasive blood pressure measurements, we used the same technique and calculations with this bedside test as well. Despite this difference in approach, the likelihood ratios were comparable. For example, we found a LLR+ for healing after 12 and 26 weeks based on the ankle pressure of 1.53 and 1.46 with a cut-off values of $>153 \mathrm{mmHg}$ and $>96.0 \mathrm{mmHg}$. Other studies found an LLR+ of $1.08(>50 \mathrm{mmHg})(29), 1.46(\geq 50 \mathrm{mmHg})$ (30), $2.52(\geq 80 \mathrm{mmHg})(30), 3.24(\geq 70 \mathrm{mmHg})(31)$ and $6.40(\geq 100 \mathrm{mmHg})(32)$. 
Although the studies with a higher threshold $(>70 \mathrm{mmHg})$ showed a higher LLR+, this effect was still small (LLR+: 2.52-3.24) to moderate (LLR+6.40), while other studies observed no change in effect based on ankle pressure measurements (LLR+: 1.08 1.46).

Similar findings are seen when comparing the found LLR+ and LLR- for toe pressure and $\mathrm{T}_{\mathrm{A}} \mathrm{O}_{2}$. Our study found LLR+ and LLR-for toe pressure and $\mathrm{T}_{\mathrm{A}} \mathrm{O}_{2}$ of $1.46,0.40$ and $1.24,0.47$ respectively. Other studies reported similar LLR+ and LLR- for toe pressure measurements. For example 1.12, 0.88 (29); 1.28, 0.33 (30); 2.47, 0.21 (31); $2.88,0.64$ (33); 4.30, 0.25 (30) and 5.00, 0.88 (33). Although the LLR are not exactly identical, the results are similar in effect and range from no-effect (LLR+: 1-2; LLR-: 0.5-1), to a small effect (LLR+: 2-5; LLR-: 0.2-0.5), comparable to our findings of noeffect (LLR+: 1.46) and a small effect (LLR-: 0.40).

When we compared our LLR+ for $\mathrm{TcpO}_{2}$ with other LLR+ values, some studies did find larger effects: LLR+ of 10.03 (33) and 5.14 (34) were found for $\mathrm{TcpO}_{2}$ thresholds $\geq 30 \mathrm{mmHg}$, indicating a moderate to large prognostic effect. However, those findings were not unanimous as other studies found lower LLR+ $(1.21$ and 2.73$)(35,36)$. This is an indication that the prognostic power of different tests are influenced by the specific patient populations and other factors such as environment and time period.

As this is the first study to investigate LLR+ and LLR- for LSCI in relation to diabetic foot ulcer healing, there are no findings for direct comparison. However, in light of the above-mentioned studies, our findings are within expected range. Despite the advantages of measuring at and around the exact ulcer location, and including both baseline perfusion values and stress-test values, $\mathrm{LSCl}$ did not result in improved prognostic likelihood ratios for ulcer healing in this cohort, in comparison to regular non-invasive blood pressure measurements.

The following limitations of this study should be considered. First, the combination of more than one prognostic test may provide more useful information on the probability of healing than a single test or test used in isolation (8). However, in the current study, we analysed the different blood pressure tests individually instead of combining them. While it is interesting to do so in a follow-up study, with the low likelihood ratios found, the benefits of combining may be small.

Second, the exclusion of patients that underwent a major amputation can be considered as another limitation. A major amputation could have been considered as endpoint, too, in addition to wound healing. This could be useful for clinicians in order to identify the patients with a higher probability of healing without revascularization to pursue a conservative approach. Also, it could be of importance to identify patients with an unacceptable high risk of a major amputation. For those patients, adequate revascularization should be a priority (8). So, amputation incidence can help in assessing the impact of disease. However, it is not necessarily a good measure of the 
quality of care and amputation incidence is partially based on the clinical choice of the attending physician (37). Therefore, we decided to focus on the healing of the diabetic foot as a biological endpoint.

Third, the follow-up period of six months and the use of two measurement moments (at 12 and 26 weeks) dichotomizes healing, rather than using the more detailed time to healing in days or weeks. This dichotomization results in diverse groups, where both short healing times ( $<4$ weeks) and long healing times ( $>20$ weeks) could end up in the same group. In further research it might be better to use time to heal (in weeks) as an endpoint for that study. This gives a better understanding and more useful parameter to get meaningful insights in the patients' healing tendency, since the time needed to heal a chronic diabetic foot ulcers usually varies a lot. We decided to use a different approach in this study because of several reasons. First, we wanted to compare our results with previous research (9). Second, dichotomization is recommended for the assessment of data in diabetic foot research (e.g. (38)). Third, diagnostic values cannot be calculated for a continuous outcome measure.

A fourth limitation of this study is that drug use and specific additional treatment of the patient (for example offloading or wound dressings) were not taken into account in this study. Where the first might influence microcirculation measurements, the latter might influence healing outcomes. However, because all patients were treated in the same centre and by the same clinicians, clinical decisions were considered similar, and therefore not accounted for in analyses. And while some drugs might affect microcirculation, no previous study on prognosis has found an effect of such drugs on likelihood ratios for prognosis (8).

Finally, it is questionable whether we can compare our results with outcomes of older studies. Although we see comparable results, it is likely that the included populations differ. Whereas in the past the majority of patients with diabetic foot ulcers had been treated in hospitals, nowadays only the more complex cases visit the hospitals or specialized care centres for diabetic foot ulcers. For future research it would be interesting to compare and validate our findings with more recent studies.

\subsection{CONCLUSION}

No association between healing of diabetic foot ulcers and microcirculation measured with LSCl or non-invasive blood pressure measurements was found. We can conclude that both types of measurements were not useful as a standalone prognostic instrument for diabetic foot ulcer healing. 


\section{REFERENCES}

1. IDF Diabetes Atlas 9th edition 2019. https://www.diabetesatlas.org/en/

2. Boulton AJ, Vileikyte L, Ragnarson-Tennvall G, Apelqvist J. The global burden of diabetic foot disease. Vol. 366, Lancet. 2005. p. 1719-24.

3. Kerr M, Rayman G, Jeffcoate WJ. Cost of diabetic foot disease to the National Health Service in England. Diabet Med. 2014 Dec;31(12):1498-504.

4. Nabuurs-Franssen MH, Huijberts MSP, Nieuwenhuijzen Kruseman AC, Willems J, Schaper NC. Health-related quality of life of diabetic foot ulcer patients and their caregivers. Diabetologia. 2005 Sep;48(9):1906-10.

5. Jupiter DC, Thorud JC, Buckley CJ, Shibuya N. The impact of foot ulceration and amputation on mortality in diabetic patients. I: From ulceration to death, a systematic review. Int Wound J. 2015;13(5):892-903.

6. Hinchliffe R, Brownrigg J, Apelqvist J, Boyko EJ, Fitridge R, Mills J, et al. IWGDF guidance on the diagnosis, prognosis and management of peripheral artery disease in patients with foot ulcers in diabetes. Diabetes Metab Res Rev. 2016;32:37-44.

7. Prompers L, Schaper N, Apelqvist J, Edmonds M, Jude E, Mauricio D, et al. Prediction of outcome in individuals with diabetic foot ulcers: Focus on the differences between individuals with and without peripheral arterial disease. The EURODIALE Study. Diabetologia. 2008;51(5):747-55.

8. Forsythe RO, Apelqvist J, Boyko EJ, Fitridge R, Hong JP, Katsanos K, et al. Performance of prognostic markers in the prediction of wound healing or amputation among patients with foot ulcers in diabetes: A systematic review. Diabetes Metab Res Rev . 2020 Mar 16;36(S1).

9. Jones DW, Wyers MC. Lower Extremity Arterial Reconstruction in Patients with Diabetes Mellitus: Principles of Treatment. In 2018. p. 327-43.

10. Spångeus A, Wijkman M, Lindström T, Engvall JE, Östgren CJ, Spångéus A, et al. Toe brachial index in middle aged patients with diabetes mellitus type 2: Not just a peripheral issue. Diabetes Res Clin Pract. 2013;100(2):195-202.

11. Faglia E, Clerici G, Caminiti M, Quarantiello A, Curci V, Morabito A. Predictive Values of Transcutaneous Oxygen Tension for Above-the-ankle Amputation in Diabetic Patients with Critical Limb Ischemia. Eur J Vasc Endovasc Surg. 2007 Jun;33(6):7316.

12. Mills JL. Open bypass and endoluminal therapy: Complementary techniques for revascularization in diabetic patients with critical limb ischaemia. In: Diabetes/Metabolism Research and Reviews. 2008.

13. Lukkari-Rautiarinen E, Lepäntalo M, Pietilä J. Reproducibility of skin blood flow, perfusion pressure and oxygen tension measurements in advanced lower limb ischaemia. Eur J Vasc Surg. 1989;3(4):345-50.

14. Mennes OA, van Netten JJ, Slart RHJ., Steenbergen W. Novel Optical Techniques for Imaging Microcirculation in the Diabetic Foot. Curr Pharm Des. 2018;24(12):1304-16.

15. Briers D. Laser Doppler, speckle and related techniques for blood perfusion mapping and imagingLaser Doppler, speckle and related techniques for blood perfusion mapping and imaging. 2001;35.

16. Boas DA, Dunn AK. Laser speckle contrast imaging in biomedical optics. J Biomed Opt. 2010;15(1):011109.

17. Roustit M, Millet C, Blaise S, Dufournet B, Cracowski JL. Excellent reproducibility of 
laser speckle contrast imaging to assess skin microvascular reactivity. Microvasc Res. 2010;80(3):505-11.

18. Millet C, Roustit M, Blaise S, Cracowski JLL. Comparison between laser speckle contrast imaging and laser Doppler imaging to assess skin blood flow in humans. Microvasc Res. 2011;82(2):147-51.

19. Mahe G, Humeau-Heurtier A, Durand S, Leftheriotis G, Abraham P. Assessment of Skin Microvascular Function and Dysfunction With Laser Speckle Contrast Imaging. Circ Cardiovasc Imaging. 2012;5(1):155-63.

20. Iredahl F, Löfberg A, Sjöberg F, Farnebo S, Tesselaar E. Non-Invasive Measurement of Skin Microvascular Response during Pharmacological and Physiological Provocations. Connes P, editor. PLoS One. 2015 Aug 13;10(8):e0133760.

21. Mennes OA, van Netten JJ, van Baal JG, Steenbergen W. Assessment of microcirculation in the diabetic foot with laser speckle contrast imaging. Physiol Meas. 2019;

22. van Netten JJ, Bus SA, Apelqvist J, Lipsky BA, Hinchliffe RJ, Game F, et al. Definitions and criteria for diabetic foot disease on behalf of the International Working Group on the Diabetic Foot. 2020;19.

23. Lipsky BA, Senneville É, Abbas ZG, Aragón-Sánchez J, Diggle M, Embil JM, et al. Guidelines on the diagnosis and treatment of foot infection in persons with diabetes (IWGDF 2019 update). Diabetes Metab Res Rev . 2020 Mar 16;36(S1):e3280.

24. Startpagina diabetische voet - Richtlijn - Richtlijnendatabase . https://richtlijnendatabase.nl/richtlijn/diabetische_voet/startpagina_diabetische_voet.h tml

25. Schaper NC, van Netten JJ, Apelqvist J, Bus SA, Hinchliffe RJ, Lipsky BA. Practical Guidelines on the prevention and management of diabetic foot disease (IWGDF 2019 update). Diabetes Metab Res Rev . 2020 Mar 1; 36(S1).

26. Forsythe RO, Apelqvist J, Boyko EJ, Fitridge R, Hong JP, Katsanos K, et al. Performance of prognostic markers in the prediction of wound healing or amputation among patients with foot ulcers in diabetes: A systematic review. Diabetes Metab Res Rev . 2020 Mar 16;36(S1):e3278.

27. Hinchliffe RJ, Forsythe RO, Apelqvist J, Boyko EJ, Fitridge R, Hong JP, et al. Guidelines on diagnosis, prognosis, and management of peripheral artery disease in patients with foot ulcers and diabetes (IWGDF 2019 update). Diabetes Metab Res Rev . 2020 Mar 20;36(S1).

28. Lavery LA, Armstrong DG, Harkless LB. Classification of diabetic foot wounds. J Foot Ankle Surg . 1996;35(6):528-31.

29. Elgzyri T, Larsson J, Thörne J, Eriksson K-FF, Apelqvist J. Outcome of Ischemic Foot Ulcer in Diabetic Patients Who Had no Invasive Vascular Intervention. Eur J Vasc Endovasc Surg. 2013;46(1):110-7.

30. Gershater MA, Löndahl M, Nyberg P, Larsson J, Thörne J, Eneroth M, et al. Complexity of factors related to outcome of neuropathic and neuroischaemic/ischaemic diabetic foot ulcers: a cohort study. Diabetologia ;52(3):398-407.

31. Wallin $\mathrm{L}, \mathrm{Björnsson} \mathrm{H}$, Stenström A. Fluorescein angiography for predicting healing of foot ulcers. Acta Orthop . $1989 ; 60(1): 40-4$.

32. Holstein P, Lassen NA. Healing of ulcers on the feet correlated with distal blood pressure measurements in occlusive arterial disease. Acta Orthop . 1980;51(16):995-1006. 
33. Kalani M, Ostergren J, Brismar K, Jörneskog G, Fagrell B. Transcutaneous oxygen tension and toe blood pressure as predictors for outcome of diabetic foot ulcers. Diabetes Care . 1999 Jan;22(1):147-51.

34. Wang Z, Hasan R, Firwana B, Elraiyah T, Tsapas A, Prokop L, et al. A systematic review and meta-analysis of tests to predict wound healing in diabetic foot . Vol. 63, Journal of Vascular Surgery. Mosby Inc.; 2016. p. 29S-36S.e2.

35. Fagher K, Katzman P, Löndahl M. Transcutaneous oxygen pressure as a predictor for short-term survival in patients with type 2 diabetes and foot ulcers: a comparison with ankle-brachial index and toe blood pressure. Acta Diabetol . 2018 Aug 1;55(8):781-8. /pmc/articles/PMC6060900/

36. Rajagopalan C, Viswanathan V, Rajsekar S, Selvaraj B, Daniel L. Diabetic foot ulcerscomparison of performance of ankle-brachial index and transcutaneous partial oxygen pressure in predicting outcome. Int J Diabetes Dev Ctries . 2018 Apr 1;38(2):179-84.

37. Jeffcoate W, Game F, Morbach S, Narres M, Van Acker K, Icks A. Assessing data on the incidence of lower limb amputation in diabetes. Diabetologia . 2021 Jun 30;64(6).

38. Jeffcoate WJ, Bus SA, Game FL, Hinchliffe RJ, Price PE, Schaper NC. Reporting standards of studies and papers on the prevention and management of foot ulcers in diabetes: required details and markers of good quality. Lancet Diabetes Endocrinol. 2016;4(9):781-8. 



\section{Semi-Automatic Tracking of Laser Speckle Contrast Images of Microcirculation in Diabetic Foot Ulcers}

O.A. Mennes, M. Selles, J.J. van Netten, J.G. van Baal, W. Steenbergen, R.H.J.A. Slart.

Diagnostics 2020; 10(12): 1054. (DOI: 10.3390/diagnostics10121054) 


\section{ABSTRACT}

Background: Foot ulcers are a severe complication of diabetes mellitus. Assessment of the vascular status of diabetic foot ulcers with Laser Speckle Contrast Imaging (LSCI) is a promising approach for diagnosis and prognosis. However, manual assessment during analysis of LSCI limits clinical applicability. Our aim was to develop and validate a fast and robust tracking algorithm for semi-automatic analysis of LSCI data.

Method: The feet of 33 participants with a diabetic foot ulcer were recorded with $\mathrm{LSCl}$, including at baseline, during the Post-Occlusive Reactive Hyperaemia (PORH) test and during the Buerger's test. Different regions of interest (ROIs) were used to measure microcirculation in different areas of the foot. A tracking algorithm was developed in MATLAB to reposition the ROIs in the LSCI scans. Manual- and algorithm-tracking of all recordings were compared by calculating the Intraclass Correlation Coefficient (ICC).

Results: The algorithm was faster in comparison to the manual approach (90sec. vs 15min.). Agreement between manual- and algorithm-tracking was good to excellent during baseline $(\mathrm{ICC}=0.896-0.984 ; \mathrm{p}<0.001)$, the PORH-test $(\mathrm{ICC}=0.790-0.960$; $p<0.001)$ and the Buerger's test (ICC $=0.851-0.978 ; p<0.001)$.

Conclusion: Resulting in a tracking algorithm that delivers assessment of $\mathrm{LSCl}$ in diabetic foot ulcers with results comparable to a labor-intensive manual approach, but with a 10 -fold workload reduction. 


\subsection{INTRODUCTION}

The incidence of diabetes mellitus increases worldwide, followed by an increasing number of related complications such as diabetic foot disease (1). It is estimated that diabetic foot disease ranks in the top ten of global burden of diseases (2). The most common aspect of this disease are diabetic foot ulcers, which are related to peripheral neuropathy and peripheral artery disease (PAD) (3). Due to peripheral neuropathy, tissue damage to the foot can go unnoticed. At the same time, PAD and capillary dysfunction cause ischemia, and impaired wound healing in case an ulcer develops $(4,5)$.

Treatment of diabetic foot ulcers relies on five major factors, including relief of pressure and protection and care of the ulcer, restoration of skin perfusion, treatment of infection, metabolic control and treatment of co-morbidities (6). To determine the need of restoration of skin perfusion, it is essential to determine the vascular status of a patient. Based on this assessment, treatment decisions such as the need for revascularization are taken. However, this vascular assessment is one of the biggest challenges in the treatment of diabetic foot ulcers (6).

The clinical standard for vascular assessment consists primarily of non-invasive assessment of blood pressure (e.g. by determining the ankle-brachial index (ABI), toebrachial index (TBI) or transcutaneous oxygen pressure (TcPO2)). However, noninvasive blood pressure measurements fail to adequately assess microcirculation in the area of the ulcer. $\mathrm{ABI}$ and $\mathrm{TBI}$ only assess macrocirculation in the lower extremities, while with $\mathrm{TCPO} 2$ the microcirculation is assessed locally, but not necessarily in or adjacent to the ulcer, and it can be influenced by other factors such as oedema or infection (7). Furthermore, the reliability of non-invasive assessment of blood pressure in people with diabetes is questionable as vascular calcification stiffens the arterial wall, making arteries poorly compressible (8).

Novel optical imaging techniques such as Laser Speckle Contrast Imaging (LSCl) are promising for the assessment of microcirculation, and not yet applied as clinical routine. $\mathrm{LSCl}$ provides real-time, non-contact imaging of the superficial microcirculation of foot tissue, and images a large tissue area (9). Therefore, LSCI could be an eligible tool for the assessment of microcirculation in patients with diabetic foot ulcers $(10,11)$.

LSCI is a technique that can image and estimate blood flow in tissue down to a depth of $1-1.5 \mathrm{~mm}$ (10). When coherent light is diffusely backscattered by a medium, it forms an interference pattern also called a speckle pattern. Moving particles in the illuminated medium will cause temporal fluctuations in the speckle pattern. Presence of more and faster moving particles results in more and faster fluctuations in the speckle pattern 
and causes a blurred speckle pattern image when imaged with an exposure time larger than the time scale of the speckle fluctuations (12-14). By quantifying the contrast of the speckles, an indication of the concentration of moving particles (which are predominantly red blood cells in the case of tissue imaging) and their speed can be made (12). Quantification of the movement of the red blood cells in the skin results in an estimation of perfusion. $\mathrm{LSCl}$ can therefore complement the currently used noninvasive blood pressure measurements to assess the level of ischemia in diabetic foot ulcers, for example directly at or adjacent to the ulcer location. Furthermore it can be performed during stress tests that provide additional clinical information, such as the Post-Occlusive Reactive Hyperaemia (PORH) test, where the peak blood flow is measured after an occlusion of the ankle, or the Buerger's test, where the leg is raised to reduce blood flow in the foot for $30-60$ seconds $(11,15,16)$.

When LSCl is applied to assess diabetic foot ulcers, different regions of interest (ROls) can be assessed simultaneously. This is useful, since both the ulcer and the ulcer edge are of particular interest, and also allows for analysis of multiple ulcers on one foot (10). Currently, ROls have to be drawn manually to analyse the measurements. This is time consuming and limits clinical applicability of LSCl. An algorithm that assists in positioning the $\mathrm{ROI}$ during the entire measurement, and that performs despite the presence of foot or leg movements, may help to overcome this drawback.

The aim of this study was to (1) develop, and (2) validate a fast and robust tracking algorithm for semi-automatic analysis of LSCI data, to improve clinical applicability of LSCl.

\subsection{MATERIALS AND METHODS}

\subsubsection{Data acquisition}

The clinical dataset was obtained as part of a larger study (17). This study was approved by a registered medical ethics committee and the study was registered in the Dutch trial register (NTR5116; 25-03-2015). Thirty-three patients with a diabetic foot ulcer participated. LSCI measurements of each ulcerated foot were performed by two operators (operator $\mathrm{A}$ and operator $\mathrm{B}$ ), resulting in a total of $66 \mathrm{LSCl}$ recordings. The recordings were obtained using a PeriCam PSI (Perimed AB, Stockholm, Sweden). Before each use the system was calibrated. During the measurements, both a PORHtest and Buerger's test were performed to amplify differences in perfusion between (critical-)ischemic and non-ischemic patients $(11,15,16)$. 
Four ellipse-shaped Regions of Interest (ROIs) were selected based on the grey scale intensity image of the LSCl scan, at three Timespans of Interest (TOls). The ROls included: (1) the ulcer, (2) the ulcer edge, (3) the toe (hallux or, when the hallux was amputated, digitus II), and (4) the entire plantar or dorsal side of the foot (the side where the ulcer was located was measured). Pixels in the background of the scan were excluded from the dataset and did not influence ROI placement or calculations of the microcirculation (Figure 1).

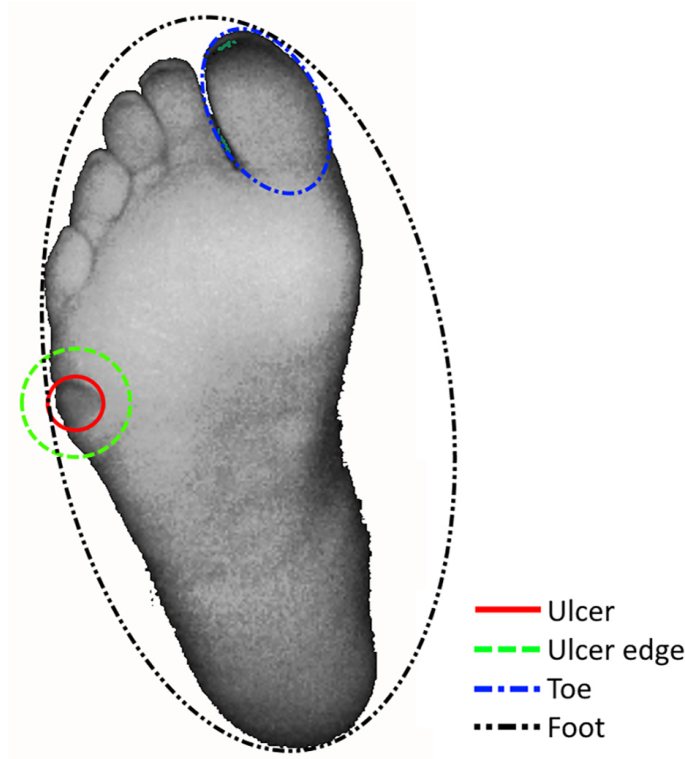

Figure 1: Placements of four Regions of Interest (ROI) of Laser Speckle Contrast Imaging scans; (1) the ulcer, (2) the ulcer edge, (3) the toe (hallux or digitus II) and (4) the entire foot (in this case the plantar side).

The TOls included: (1) baseline, (2) the moment of peak perfusion during the PORHtest and (3) the Buerger's test (Figure 2). In case no well-defined peak perfusion was found during the PORH-test, the maximum measured value when the perfusion stops increasing was used to define TOI 2. All ROls were manually positioned for the first frame. 
TOI 2

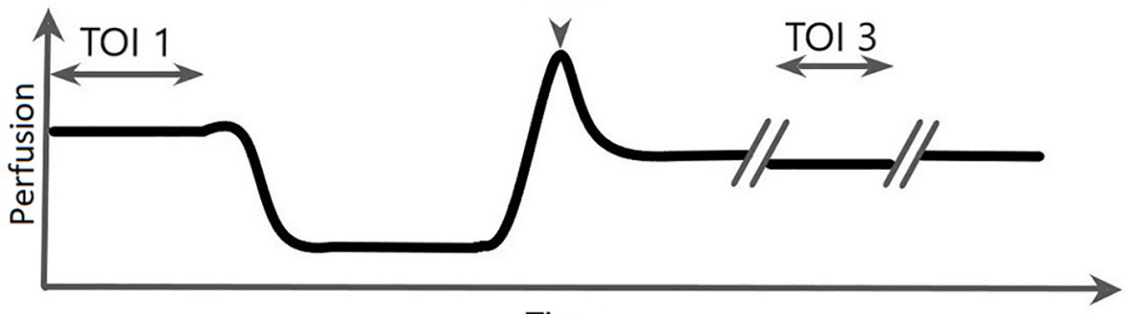

Time

Figure 2: Placements of Timespans of Interest (TOI) of Laser Speckle Contrast Imaging scans. TOI 1: baseline, TOI 2: Post-Occlusive Reactive Hyperaemia test, peak perfusion after reperfusion. TOI 3: Buerger's test. //: Short break in scan to reposition the foot before and after Buerger's test.

In manual tracking, ROls were manually repositioned for each frame during the scan, to compensate for movement of the foot during measurement. For algorithm tracking, the ROls from the first frame were used to automatically reposition the ROls during the rest of the scan when required. A second set of ROls was drawn before the Buerger's test and automatically used for this TOI. The shape of the foot was detected using Canny edge detection (18) as implemented in MATLAB's Image Processing Toolbox (19) for all frames of the recording. Mean perfusion values for each ROI during each TOI were calculated and used for data analysis, and were computed with Pimsoft software (Perimed AB, Stockholm, Sweden).

\subsubsection{Algorithm development}

An algorithm was developed with custom-written code in MATLAB (The MathWorks inc.; Natic; USA). Following manual assessment of ROls in the first frame, the ROls in frames $[2, n]$ were computed using the Iterative Closest Point algorithm (20) (ICP) as implemented in MATLAB's Computer Vision System Toolbox (21). ICP computes the transformation matrix between two images to align two images. The transformation matrix consists of a rotational and translational component.

Likewise, the transformation matrices of frame 1 to frames $[2, n]$ were computed based on the shape of the foot, resulting in $n-1$ transformations. Application of the corresponding transformation matrices to the ROls as defined in frame 1 resulted in the ROls in the frames [2,n] (Figure 3). 


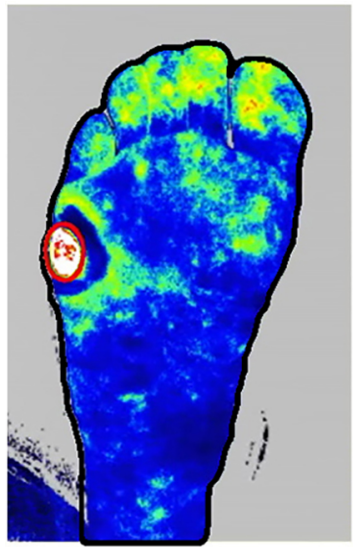

Frame 1

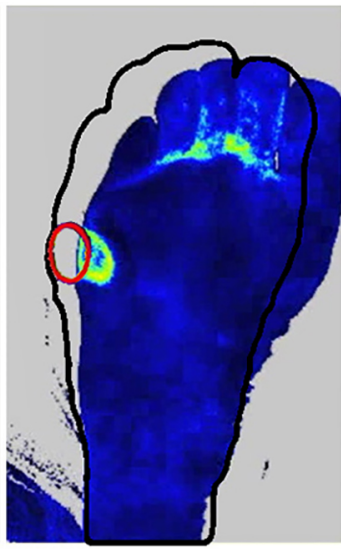

Frame 2

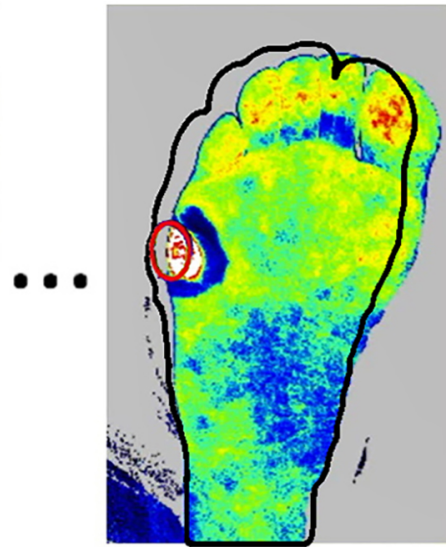

Frame $n$

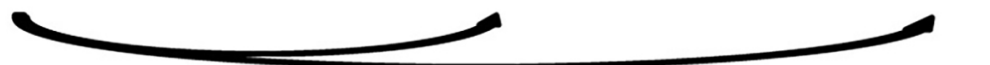

Compute transformations

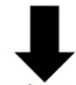

Apply transformations to ROI in frame 1

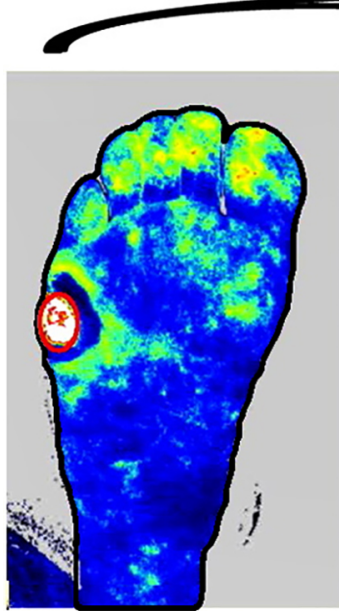

Frame 1, with ROI

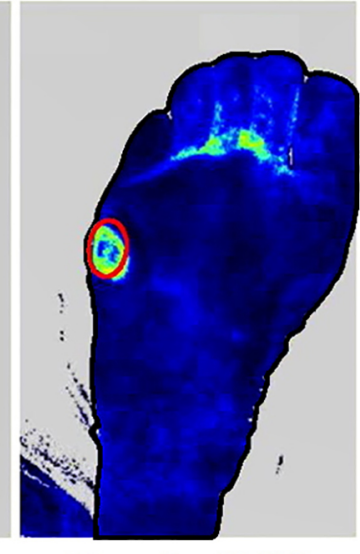

Frame 2, with ROI

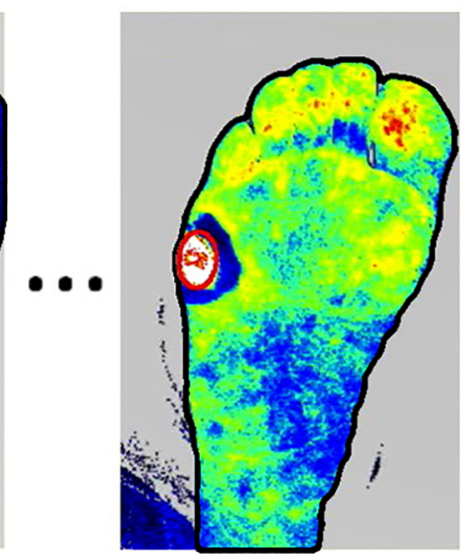

Frame $n$, with ROI

Figure 3: Principle of the region of interest (ROI) tracking. The transformation from frame 1 towards the other frames is computed based on the shape of the foot in both frames. The ROI in the frames $[2, n]$ is computed by application of the transformation from frame 1 to frame $n$ on the initial ROI. If, as shown, the foot moves to right, the ROI follows correct placement at the ulcer location. 
To prevent erroneous calculations of ROls due to static artefacts in the background (such as the examination bench), the region without static artefacts was once selected manually in frame 1 of the recording (Figure 4). Consequently, the region without static artefacts was used to calculate the transformation matrices and compute the ROIs in the frames $[2, n]$.

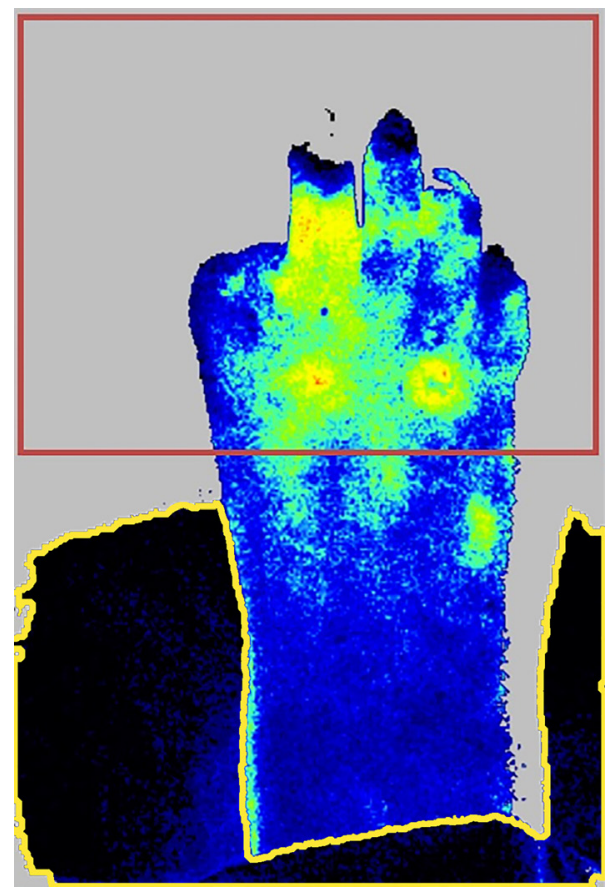

Figure 4: Example of a Laser Speckle Contrast Imaging recording with static artefacts. The red box defines the selected area of the image that was used for ROI tracking. Static artefacts (e.g. the examination bench and pressure band) are highlighted by the yellow outline and were ignored when calculating the transformation matrices.

Because of the repositioning of the foot during Buerger's test, the shape of the imaged foot changed, with the foot becoming a non-rigid object (Figure 5). Therefore, a second set of ROls was manually drawn for the first frame of the Buerger's test. The transformation matrices for the Buerger's test frames were reset and new transformations and $\mathrm{ROI}$ repositioning were used in this TOI. 

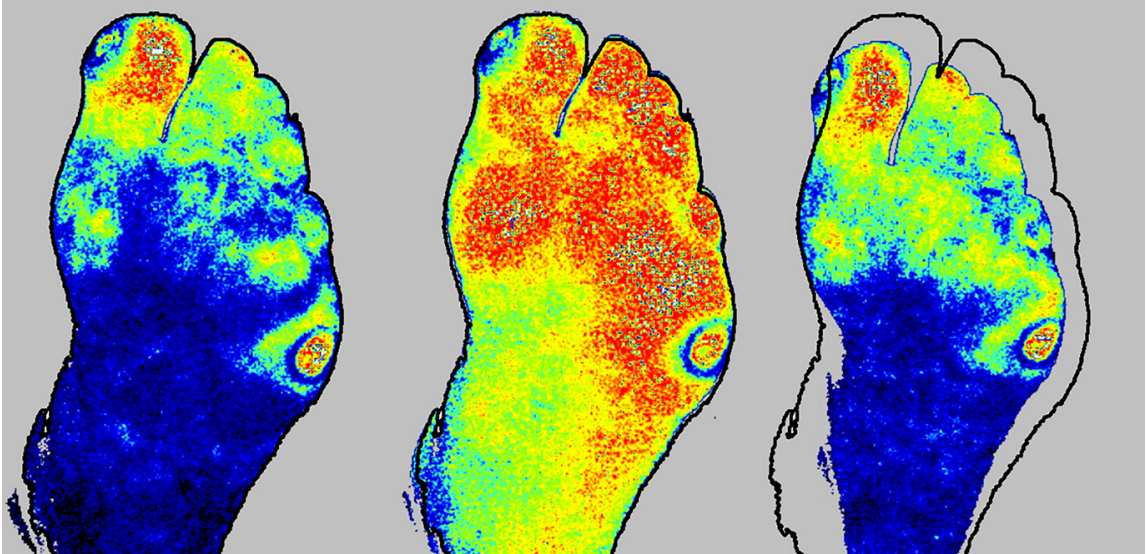

Figure 5: ROI tracking during baseline measurement (left), Post-Occlusive Reactive Hyperaemia test (middle) and Buerger's test (right). Here, the ROI during Buerger's test was erroneous, illustrating the need for redrawing the ROls.

The perfusion was calculated in MATLAB identical to perfusion calculations as done by the Pimsoft software (Perimed AB, Stockholm, Sweden). The equations as stated below were provided by Perimed. The intensity and variance were used to calculate the perfusion in the ROIs for all TOls. First, the mean intensity $\langle I\rangle$ and the standard deviation $\sigma$ in all four ROIs were calculated for TOI 1, TOI 2 and TOI 3.

Then, the mean contrast was computed by (22)

$$
K=\beta \frac{\sigma}{\langle I\rangle}(\text { eq. } 1)
$$

with $\beta$ as the coherence factor. The coherence factor is instrument-specific and dependent on calibration and ensures that $K=1$ for static objects. The coherence factor was obtained by exporting the recordings to MATLAB.

From the mean contrast the mean perfusion was calculated as

$$
P=\mathrm{k}\left(\frac{1}{K}-1\right)(\text { eq. } 2)
$$

with $\mathrm{k}$ the signal gain. Similar to the coherence factor $\beta$, the signal gain is also instrument-specific and ensures that an instrument, after calibration, measures the same perfusion value when measuring the same tissue. The calibration for both the signal gain $(k)$ and coherence factor $(\beta)$ was performed by measuring a zero-perfusion area and a colloidal suspension of polystyrene particles to set the $\mathrm{LSCl}$ values on $0 \pm 5$ perfusion units (PU) and 250 \pm 5 PU respectively. 


\subsubsection{Algorithm validation}

All 66 LSCl scans were recorded by two operators (operator A and B). All scans were manually analysed by two assessors (assessor 1 [same as operator A] and assessor 2) and all scans were analysed by the tracking algorithm. Time required for each analyses (manual or algorithm) was measured by performing 10 measurements and calculating the average time needed for the manual input. Performing these assessments resulted in the mean perfusion for all four ROls during all three TOls. By subjecting the two datasets of operator $A$ and $B$ to the analyses of the two different assessors and the algorithm, this process resulted in a total of six datasets (Figure 6).

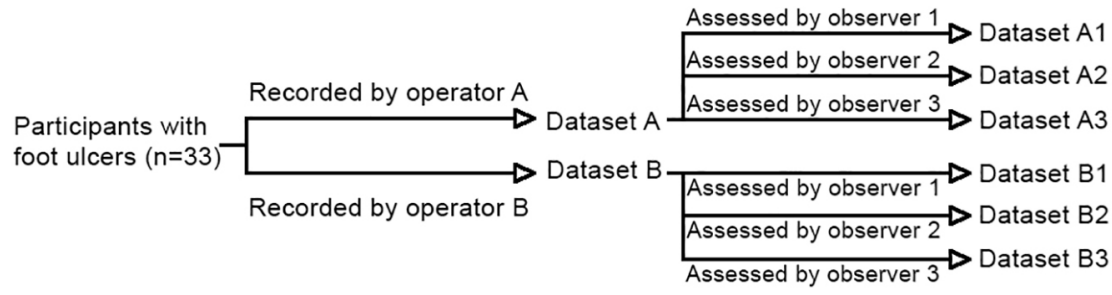

Figure 6: Schematic overview of datasets

To determine the intra-assessor variation, Intraclass Correlation Coefficient (ICC) with a two-way random effects model was calculated between the different datasets. The definition used for ICC testing was absolute agreement where values less than 0.5 are indicative of poor reliability, values between 0.5 and 0.75 are moderate, between 0.75 and 0.9 are good and greater than 0.90 are indicative of excellent reliability (23). Statistical analyses were performed using SPSS version 23.0 (SPSS Inc. Chicago, IL, USA).

\subsection{RESULTS}

\subsubsection{Algorithm performance}

The tracking algorithm was able to analyse all LSCI scans. No additional ROI drawing apart from in the first frame of the baseline measurement and Buerger's test was needed. The labour time of manual selection of all ROls in both frames was approximately 90 seconds and the mean analyses duration of the algorithm was 185 seconds. This was faster than the manual assessment of an entire scan, that took on average 15 minutes per scan.

The algorithm was capable of repositioning the ROls in all scans. The algorithm correctly repositioned the ROls in the majority of the cases (Video 1A-C; Figure 7). The 
quality of the repositioning and the analyses of the $\mathrm{LSCl}$ scan varied within and between different scans. In 5.1\% (40 of 792) of all ROI placements, some suboptimal ROls were drawn resulting in a measured PU value 10\% higher or lower than measured during the manual approach. Frequency of those suboptimal ROls was comparable between baseline ( $n=15,5.7 \%), \operatorname{PORH}(n=15,5.7 \%)$ and Buerger's test $(n=13,4.9 \%)$.
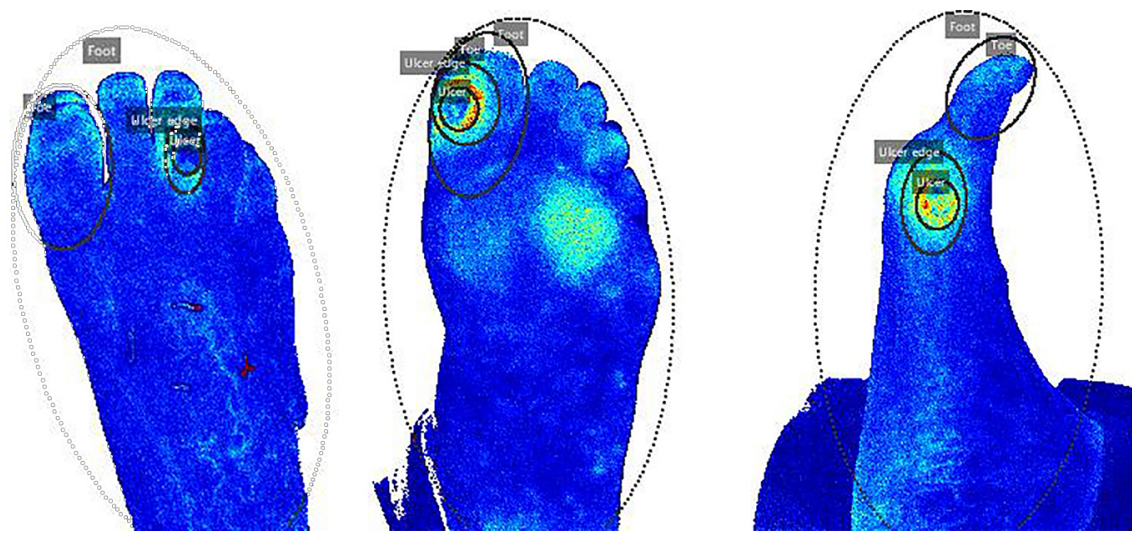

Video 1A-C: Three curated videos of diabetic feet showing the ROI repositioning during baseline, post occlusive reactive hyperaemia test and Buerger's test measurements. Available online in the electronic version of this article. 


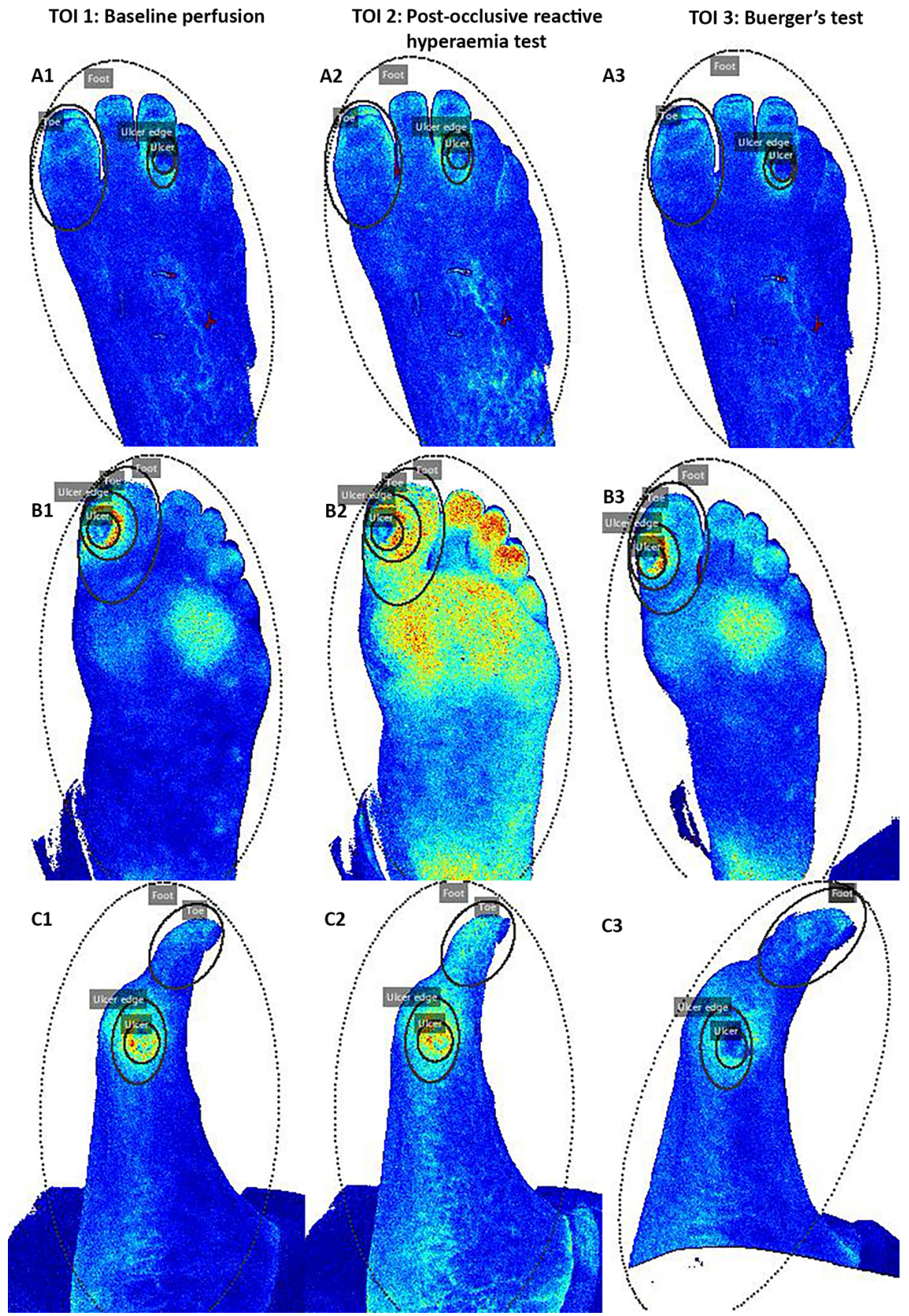

Figure 7 Left: ROI tracking during baseline measurement, Middle: Post occlusive reactive hyperaemia test and Right: Buerger's test for three different patients (A (plantar view), B (plantar view), $\mathrm{C}$ (medial view)). The ROI during Buerger's test was manually repositioned for the first frame of the test. 


\subsubsection{Algorithm validation}

The ICCs were good to excellent for all comparisons between manual and algorithm tracking, and between the two manual trackings (Figure 8, Table 1, Table 2).
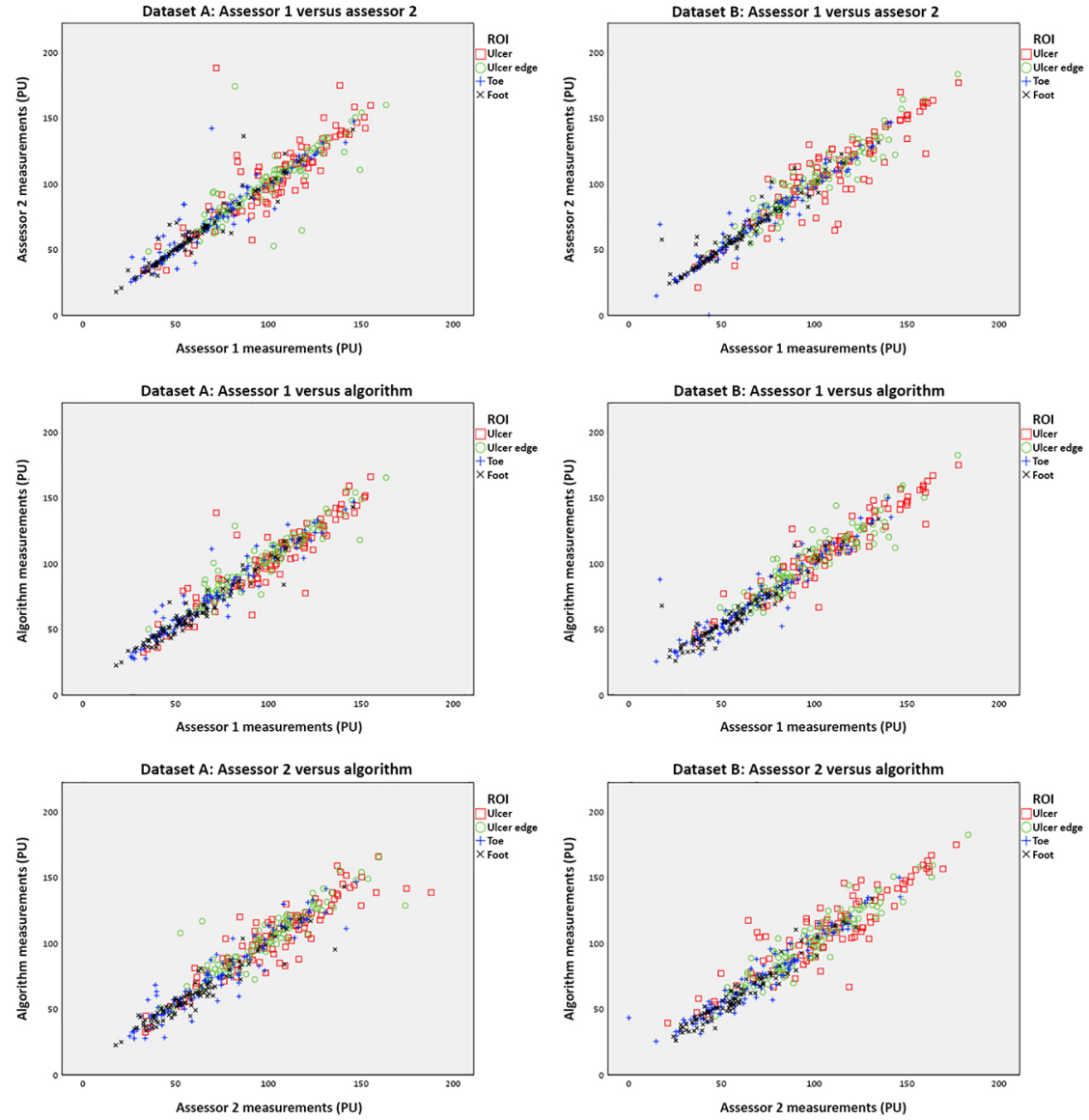

Figure 8: Scatterplots of the measurements between assessors and the algorithm. Perfusions is shown in Perfusion Units (PU) for all scans during baseline measurements and different stress tests. Recordings were obtained with Laser Speckle Contrast Imaging by operator A (Dataset A) and operator B (Dataset B) and analysed by two assessors and the algorithm. Assessors 1 and 2 and the algorithm were compared to each other for different regions of interest (ROI).

In dataset $A$, the ICCs between both assessors and the algorithm were good to excellent (ICC $=0.790-0.978$ ) and higher than the ICCs between both human assessors (ICC=0.628-0.988). Baseline ICCs (ICC=0.896-0.975) were comparable to stress-test ICCs (POHR: ICC=0.790-0.952; Buerger's-test: ICC=0.894-0.978) (Table 1). 
In dataset B, the ICCs between both assessors and the algorithm were good to excellent (ICC $=0.851-0.984)$ and comparable to the ICCs between both human assessors (ICC=0.865-0.992). Baseline ICCs (ICC=0.912-0.984) were higher compared to stress test ICCs (POHR: ICC $=0.865-0.960$; Buerger's test: ICC $=0.851$ 0.953) (Table 1).

Table 1: Intraclass Correlation Coefficients (ICC) of assessment of Laser Speckle Contrast Imaging with two Regions of Interest placements before Baseline and Buerger's test measurements.

\begin{tabular}{|c|c|c|c|c|c|c|}
\hline \multirow[t]{2}{*}{ Characteristics } & \multicolumn{3}{|c|}{ Dataset A } & \multicolumn{3}{|c|}{ Dataset B } \\
\hline & $\begin{array}{c}\text { Assessor } \\
1 \text { vs. } 2\end{array}$ & $\begin{array}{l}\text { Assessor } \\
1 \text { vs. Alg. }\end{array}$ & $\begin{array}{l}\text { Assessor } \\
2 \text { vs. Alg. }\end{array}$ & $\begin{array}{c}\text { Assessor } \\
1 \text { vs. } 2\end{array}$ & $\begin{array}{l}\text { Assessor } \\
1 \text { vs. Alg. }\end{array}$ & $\begin{array}{l}\text { Assessor } \\
2 \text { vs. Alg }\end{array}$ \\
\hline \multicolumn{7}{|l|}{ Baseline } \\
\hline Ulcer & 0.936 & 0.968 & 0.953 & 0.950 & 0.984 & 0.954 \\
\hline Ulcer edge & 0.936 & 0.975 & 0.896 & 0.966 & 0.969 & 0.963 \\
\hline Toe & 0.983 & 0.953 & 0.942 & 0.992 & 0.963 & 0.970 \\
\hline Foot & 0.988 & 0.916 & 0.897 & 0.959 & 0.912 & 0.935 \\
\hline \multicolumn{7}{|l|}{ PORH } \\
\hline Ulcer & 0.628 & 0.790 & 0.861 & 0.914 & 0.949 & 0.865 \\
\hline Ulcer edge & 0.706 & 0.846 & 0.894 & 0.932 & 0.955 & 0.952 \\
\hline Toe & 0.828 & 0.910 & 0.883 & 0.917 & 0.874 & 0.949 \\
\hline Foot & 0.883 & 0.952 & 0.901 & 0.903 & 0.905 & 0.960 \\
\hline \multicolumn{7}{|l|}{ Buerger's test } \\
\hline Ulcer & 0.932 & 0.954 & 0.918 & 0.865 & 0.913 & 0.851 \\
\hline Ulcer edge & 0.912 & 0.945 & 0.894 & 0.953 & 0.869 & 0.923 \\
\hline Toe & 0.970 & 0.978 & 0.962 & 0.931 & 0.951 & 0.937 \\
\hline Foot & 0.980 & 0.958 & 0.973 & 0.980 & 0.953 & 0.952 \\
\hline
\end{tabular}

Legend: The recordings were observed by assessor 1 and 2 and the algorithm (Alg). The ICCs were calculated for the three Timespans of Interest (Baseline perfusion, Post-Occlusive Reactive Hyperaemia (PORH) and Buerger's test) and the four Regions of Interest (Ulcer, Ulcer edge, Toe and Foot). Note: $p \leq 0.001$ for all ICCs.

When no additional ROIs were drawn on the first frame of the Buerger's test, the ICCs for the Buerger's test decreased, particularly against the algorithm (range: 0.5320.980).

\subsection{DISCUSSION}

We aimed to develop and validate a fast and robust tracking algorithm for analysis of $\mathrm{LSCl}$ data of diabetic foot ulcers, to improve clinical applicability of $\mathrm{LSCl}$ in vascular assessment in diabetic foot disease. We developed an algorithm that required minimal manual input and was able to process all LSCI data. This algorithm can do this with an approximately 10 -fold workload reduction compared to the current manual approach with software from the manufacturer, and demonstrated good to excellent agreement with this current standard approach. This shows that the algorithm achieves human- 
like performance for the assessment of LSCI data of diabetic foot disease. Currently the time-consuming analysis of $\mathrm{LSCl}$ data for the assessment of diabetic foot ulcers limits its clinical applicability; the developed algorithm is fast enough to overcome this.

While we advocate implementation of the algorithm in clinical practice, it should be noted that some of the ROls were repositioned suboptimally by the algorithm. Although the incidence of these suboptimal ROls was low, inspection of the ROls yielded by the algorithm is encouraged to examine if the ROls are repositioned correctly. Besides rigid registration, several nonrigid registration algorithms have been developed. B-spline based nonrigid registration has shown to be successful in infrared thermography of the diabetic foot (24). This approach might reduce the suboptimal ROIS, and might also eliminate the required redefinition of ROIs at the first frame of Buerger's test, further minimizing the need for human input. On the other hand, non-rigid registration is generally more time-consuming, prolonging the duration of analysis as the registration has to be performed for all frames of the LSCI recording. This needs to be investigated in future research.

The optimum analysis method of LSCl data would be a fully automated one, by automatic detection of the ulcer in the first frame. This would eliminate erroneous measurements due to human intervention and would potentially also speed up the process of analysis even further. Promising results have been shown using deep learning for the automatic segmentation of diabetic foot ulcers $(25,26)$. However, these results were achieved using RGB images, not grey scale LSCI intensity data. Either automatic detection of ulcers in LSCl perfusion data or mapping of the ulcer segmentations from RGB images to the LSCI data should be developed to make this automatic segmentation approach useful for ulcer assessment using LSCI. We think it would be easier to use RGB images of the foot instead of the grey scale intensity data or the measured perfusion data, because using RGB images allows other factors such as the colour (for example redness) of the skin to also be taken into account. Automatic detection of ulcers might also facilitate more accurate assessment of the ulcer and ulcer edge, following their real contours. We used ellipse-shaped ROls, whereas automatic detection can draw along the ulcer and along the edge of the ulcer.

There are some limitations to this study. First of all, the semi-automatic algorithm still requires human input, at the start of the LSCI recording and at the first frame of the Buerger's test. During this study, one researcher provided the input that was needed for the algorithm. Future research should investigate the inter-rater reliability when different researchers or clinicians provide the input for the algorithm. Second, in this study, we only investigated the mean PU values calculated for each ROI and compared those values with each other to measure the ICC because this is clinically most relevant. Given the complex pattern and perfusion hotspots in and around the ulcer, it makes sense that the ROls were positioned correctly if the measured PU was 
comparable with the human measurements. However, the exact location and size of the ROI placements were not compared with each other. This could be interesting for further research. Third, a possible learning curve of the assessor to analyse the data and position the different ROls was not taken into account in this study. The time needed for the manual placement of the ROls was based on the last measured patients. This time might be further reduced if the assessor has more experience with the data and the positioning of different ROIs in the LSCI scans.

$\mathrm{LSCl}$ is currently being investigated in diabetic foot disease as well as several other medical fields such as rheumatology, dermatology, ophthalmology, neurology and gastro-intestinal surgery (14). Also, in these fields, ROls at several time points or time spans are used for assessment of perfusion and tracking of the ROls might be useful. Application of the algorithm needs to be assessed in those fields. However, it should be noted that our algorithm is not useful in all fields. For instance, ROI tracking for LSCI assessment of cerebral blood flow requires different registration approaches (27). As every application of LSCI may require a different approach to address movement of ROls, this should be evaluated per application.

\subsection{CONCLUSIONS}

The developed tracking algorithm for analysis of LSCI data of diabetic foot ulcers has a good to excellent inter-rater reliability in comparison to the current standard of manual assessment. The algorithm shows a 10-fold workload reduction compared the manual approach, and may improve clinical applicability of LSCI for the assessment of diabetic foot disease. 


\section{REFERENCES}

1. Lipsky BA, Berendt AR, Cornia PB, Pile JC, Peters EJ, A.D. Infectious Diseases Society of America clinical practice guideline for the diagnosis and treatment of diabetic foot infections. Clin. Infect. Dis. 2012, 54, e132-e173.

2. Lazzarini, P.A.; Pacella, R.E.; Armstrong, D.G.; van Netten, J.J. Diabetes-related lowerextremity complications are a leading cause of the global burden of disability. Diabet. Med. 2018,

3. Fowler, M.J. Microvascular and macrovascular complications of diabetes. Clin. Diabetes 2011,

4. Cicco, G.; Giorgino, F.; Cicco, S. Wound healing in diabetes: Hemorheological and microcirculatory aspects. In Proceedings of the Advances in Experimental Medicine and Biology; 2011.

5. Hasan, R.; Firwana, B.; Elraiyah, T.; Domecq, J.P.; Prutsky, G.; Nabhan, M.; Prokop, L.J.; Henke, P.; Tsapas, A.; Montori, V.M.; et al. A systematic review and meta-analysis of glycemic control for the prevention of diabetic foot syndrome. J. Vasc. Surg. 2016.

6. Bakker, K.; Apelqvist, J.; Lipsky, B.; Van Netten, J.; International Working Group on the Diabetic foot The 2015 IWGDF Guidance documents on prevention and management of foot problems in diabetes: development of an evidence-based global consensus. Diabetes. Metab. Res. Rev. 2016, 32, 2-6.

7. Wang, Z.; Hasan, R.; Firwana, B.; Elraiyah, T.; Tsapas, A.; Prokop, L.; Mills, J.L.; Murad, M.H. A systematic review and meta-analysis of tests to predict wound healing in diabetic foot. J. Vasc. Surg. 2016.

8. Aerden, D.; Massaad, D.; Von Kemp, K.; Van Tussenbroek, F.; Debing, E.; Keymeulen, B.; Van Den Brande, P. The ankle-brachial index and the diabetic foot: A troublesome marriage. Ann. Vasc. Surg. 2011,

9. Kazmi, S.M.S.; Richards, L.M.; Schrandt, C.J.; Davis, M.A.; Dunn, A.K. Expanding applications, accuracy, and interpretation of laser speckle contrast imaging of cerebral blood flow. J. Cereb. Blood Flow Metab. 2015.

10. Mennes, O.A.; van Netten, J.J.; Slart, R.H.J..; Steenbergen, W. Novel Optical Techniques for Imaging Microcirculation in the Diabetic Foot. Curr. Pharm. Des. 2018, 24, 1304-1316,

11. Roustit, M.; Millet, C.; Blaise, S.; Dufournet, B.; Cracowski, J.L. Excellent reproducibility of laser speckle contrast imaging to assess skin microvascular reactivity. Microvasc. Res. 2010, 80, 505-511,

12. Boas, D.A.; Dunn, A.K. Laser speckle contrast imaging in biomedical optics. J. Biomed. Opt. 2010, 15, 011109,

13. Briers, D.; Duncan, D.D.; Hirst, E.; Kirkpatrick, S.J.; Larsson, M.; Steenbergen, W.; Stromberg, T.; Thompson, O.B. Laser speckle contrast imaging: theoretical and practical limitations. J. Biomed. Opt. 2013, 18, 066018,

14. Heeman, W.; Steenbergen, W.; van Dam, G.M.; Boerma, E.C. Clinical applications of laser speckle contrast imaging: a review. J. Biomed. Opt. 2019, 24, 1,

15. Humeau-Heurtier, A.; Guerreschi, E.; Abraham, P.; Mahe, G. Relevance of laser doppler and laser speckle techniques for assessing vascular function: State of the art and future trends. IEEE Trans. Biomed. Eng. 2013, 60, 659-666,

16. Iredahl, F.; Löfberg, A.; Sjöberg, F.; Farnebo, S.; Tesselaar, E. Non-Invasive Measurement of Skin Microvascular Response during Pharmacological and Physiological Provocations. PLoS One 2015, 10, e0133760, 
17. Mennes, O.A.; van Netten, J.J.; van Baal, J.G.; Steenbergen, W. Assessment of microcirculation in the diabetic foot with laser speckle contrast imaging. Physiol. Meas. 2019

18. Canny, J. A Computational Approach to Edge Detection. IEEE Trans. Pattern Anal. Mach. Intell. 1986,

19. MATLAB Image Processing Toolbox Release 2018b, The Mathworks, Inc., Natick, Massachusetts, United States.

20. Besl, P.J.; McKay, N.D. A Method for Registration of 3-D Shapes. IEEE Trans. Pattern Anal. Mach. Intell. 1992,

21. MATLAB Computer Vision System Toolbox Release 2018b, The Mathworks, Inc., Natick, Massachusetts, United States.

22. Vaz, P. G.; Humeau-Heurtier, A.; Figueiras, E.; Correia, C.; Cardoso, J. Laser speckle imaging to monitor microvascular blood flow: a review. IEEE reviews in biomedical engineering. 2016, 9, 106-120.

23. Koo, T.K.; Li, M.Y. A Guideline of Selecting and Reporting Intraclass Correlation Coefficients for Reliability Research. J. Chiropr. Med. 2016,

24. Liu, C.; van Netten, J.J.; van Baal, J.G.; Bus, S.A.; van der Heijden, F. Automatic detection of diabetic foot complications with infrared thermography by asymmetric analysis. J. Biomed. Opt. 2015,

25. Ohura, N.; Mitsuno, R.; Sakisaka, M.; Terabe, Y.; Morishige, Y.; Uchiyama, A.; Okoshi, T.; Shinji, I.; Takushima, A. Convolutional neural networks for wound detection: The role of artificial intelligence in wound care. J. Wound Care 2019,

26. Goyal, M.; Reeves, N.D.; Rajbhandari, S.; Spragg, J.; Yap, M.H. Fully convolutional networks for diabetic foot ulcer segmentation. In Proceedings of the 2017 IEEE International Conference on Systems, Man, and Cybernetics, SMC 2017; 2017.

27. Richards, L.M.; Towle, E.L.; Fox, D.J.; Dunn, A.K. Intraoperative laser speckle contrast imaging with retrospective motion correction for quantitative assessment of cerebral blood flow. Neurophotonics 2014, 



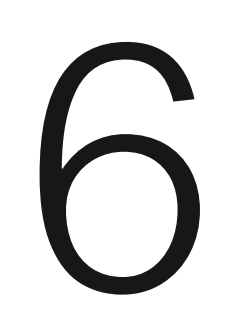

\section{Assessing the microcirculation of the plantar side of the foot with laser speckle contrast imaging during endovascular revascularisation procedures in patients with peripheral arterial occlusive disease}

O.A. Mennes, B. Wermelink, J.G. van Baal, W. Steenbergen, R.H.Geelkerken, on behalf of the PAMiPAD study group*. Submitted to European Journal of Vascular \& Endovascular Surgery

* S.H. Aarnink, R. Beuk, M. Brusse-Keizer, M. Haalboom, R.R. Kruse, S. Manohar, R. Meerwaldt, T. Menting, B.L. Reichman, R.H.J.A. Slart, E.M. Willigendael 


\section{ABSTRACT}

Background: Patients with peripheral arterial disease have a risk of developing chronic limb threatening ischemia (CLTI). Prognosis of CLTI is poor unless successful revascularisation is performed. By introducing additional peroperative assessment of the microcirculation, the impact and effect of the revascularisation could be assessed and ultimately improve the clinical outcome. Laser speckle contrast imaging (LSCI) has the potential to assess the microcirculation peroperatively, although evidence regarding the clinical applicability and accuracy is currently unclear. Aim of this study was to determine the inter-observer variation of LSCl-scans and study the potential clinical applicability of the LSCI parameters in patients with CLTI.

Methods: A prospective, exploratory, single centre, cohort study was conducted. 30 patients with CLTI that received a revascularisation procedure were included. Peroperatively a LSCl-scan was performed. Predefined regions of interest and time periods of interest were manually drawn to measure the perfusion. Inter-observer variation was determined and changes in perfusion were related to changes in clinical parameters. Patients had a clinical follow-up of 6 months.

Results: The inter-observer variation between two observers was good to excellent (0.825-1.000). After revascularisation mean peroperative $\mathrm{LSCl}$ perfusion values increased from $45.8 \pm 20.3 \mathrm{PU}$ to $66.6 \pm 30.5 \mathrm{PU}$. Also there was a significant improvement in Rutherford classification ( $4.5 \pm 0.5$ vs $2.2 \pm 2.3, p<0.001$ ), global anatomic staging system classification (aortoiliac $0.97 \pm 0.87$ vs $0 \pm 0, p<0.001$; femorocrural $1.07 \pm 0.92$ vs $0.1 \pm 0.41, p<0.001)$, $A B I(53.7 \pm 32.1 \mathrm{mmHg}$ vs $79.5 \pm 35.1 \mathrm{mmHg}$, $p=0.024)$ and walking distance $(1,7(<500 m)$ vs $2.8(>500 m), p=<0.001)$. Wound healing was reached in 8 out of 13 patients with a mean time to wound healing of $78 \pm 31$ days. Correlations between LSCI perfusion values and clinical outcome parameters were low.

Conclusions: The ICC indicates that different observers can analyse LSCI data without conflicting interpretations. Significant improvement was found between pre and postrevascularisation LSCI perfusion values. Correlation between LSCI perfusion values and clinical outcome was poor although the majority of patients showed corresponding results between clinical outcome and LSCI perfusion values. 


\subsection{INTRODUCTION}

In peripheral arterial disease (PAD) macrovascular lesions reduce the outflow perfusion. The microcirculation and nutritive blood flow to tissue becomes impaired, ultimately resulting in chronic limb threatening ischemia (CLTI), with symptoms such as ischemic rest pain or non-healing wounds (1). Treatment for PAD is limited to cardiovascular risk management, including lifestyle changes and medication, supervised exercise therapy, endovascular, hybrid or open revascularisation procedures. For patients with CLTI the prognosis of the leg is poor unless successful revascularisation is performed (2). Endovascular revascularisation procedures are performed under fluoroscopic guidance to visualise the peroperative impact of the procedure on the macrocirculation. Unfortunately, fluoroscopy lacks the ability to visualise the impact of the procedure on the microcirculation (3), while restoring the microcirculation plays a pivotal role in CLTI and wound healing (4). By introducing an additional peroperative assessment tool of the microcirculation, the impact and effect of the revascularisation could be assessed in real-time. This enables the surgical team to timely extend or adjust the revascularisation procedure if needed, and ultimately improve (prediction of) the clinical outcome.

Laser speckle contrast imaging (LSCI) is an imaging technique of the microcirculation of the outer layer of the skin, down to a depth of $0.6 \mathrm{~mm}(5-7)$. LSCl is a contactless, non-invasive, optical imaging technique that generates microvascular blood flow maps (8). Laser light scatters from the illuminated tissue surface, creating an interference pattern, a so-called speckle pattern, this pattern is recorded with a digital camera. Speckle patterns continuously change by the movement of erythrocytes in the tissue. Recording those speckle patterns with a camera with an integration time longer than the time scale of the speckle fluctuations will result in blurring of the recorded images (9). Higher velocity or higher concentration of moving erythrocytes increases this blurring. By analysing the speckle contrast of the recorded images, blood flow maps representing the superficial microcirculation are instantaneously generated and expressed in instrument specific arbitrary perfusion units (PU) (10).

Although $\mathrm{LSCl}$ is a stable and reproducible technique that can measure the microcirculation (10), the applicability to assess the microcirculation during revascularisation procedures and the relation to clinical outcome parameters in CLTI patients is not studied yet. In this study we use LSCI peroperatively in a hybrid operating theatre (HOT) to measure the effect of the macrovascular revascularisation on the microcirculation of the plantar foot in real-time and relate this with short term clinical outcome. Our aim is to determine the inter-observer variation of $\mathrm{LSCl}$ scans and study the potential clinical applicability of the LSCI parameters in patients with CLTI. 


\subsection{METHODS}

For this prospective, exploratory, single centre, cohort study, patients with CLTI were included from the vascular department of Medisch Spectrum Twente, Enschede, the Netherlands. Approval of the medical research ethics committee was acquired (NL66041.044.18) for inclusion of 30 patients. The study was registered in the Dutch Trial Register (NTR7440; 30-05-2018). All patients provided written informed consent prior to study participation.

\subsubsection{Patient characteristics}

Between February 2019 and September 2019, 30 consecutive CLTI patients were included. Inclusion criteria; patients were 18 years or older, had a Rutherford classification 4, 5 or 6 and first revascularisation procedure under general anesthesia in the HOT. Exclusion criteria; patients with multiple drug resistance bacteria to avoid contamination of any study materials, tattoo at the plantar side of the foot, toe- or forefoot amputation or previous vascular interventions in the ipsilateral lower limb. An overview of all the patient characteristics and general health status, presented in accordance with the reporting standards of the Society for Vascular Surgery for endovascular treatment of chronic lower extremity peripheral artery disease (11) (Table 1).

Table 1: Characteristics of 29 PAOD patients with LSCI microcirculation assessment during revascularisation of the limb

\begin{tabular}{|c|c|}
\hline Categories/grade-Description & Mean \pm SD; N (\%) \\
\hline Age & $71.2 \pm 9.6$ \\
\hline \multicolumn{2}{|l|}{ Sex } \\
\hline 0 - Male & $17(58.6 \%)$ \\
\hline 1 - Female & $12(41.4 \%)$ \\
\hline BMI & $25.6 \pm 4.9$ \\
\hline \multicolumn{2}{|l|}{ Diabetes • } \\
\hline 0 - None & $20(69 \%)$ \\
\hline 1 - Not requiring insulin & $1(3.4 \%)$ \\
\hline 2 - Controlled by insulin & $8(27.6 \%)$ \\
\hline 3 - Type 1 or uncontrolled & $0(0 \%)$ \\
\hline \multicolumn{2}{|l|}{ Tabaco use } \\
\hline 0 - None or remote (>10 years) & $10(34.5 \%)$ \\
\hline 1 - Quit $1-10$ years ago & $9(31 \%)$ \\
\hline 2 - Current within the last year & $10(34.5 \%)$ \\
\hline \multicolumn{2}{|l|}{ Hypertension } \\
\hline 0 - None & $9(31 \%)$ \\
\hline 1 - Controlled with 1 drug & $8(27.6 \%)$ \\
\hline 2 - Controlled with 2 drugs & $7(24.1 \%)$ \\
\hline 3 - Requiring $>2$ drugs or uncontrolled & $5(17.2)$ \\
\hline \multicolumn{2}{|l|}{ Renal status } \\
\hline 0 - Normal & $8(27.6 \%)$ \\
\hline 1 - Evidence of renal disease, GFR $>90 \mathrm{~mL} / \mathrm{min} / 1.73 \mathrm{~m}^{2}$ & $0(0 \%)$ \\
\hline 2 - GFR $60-89 \mathrm{~mL} / \mathrm{min} / 1.73 \mathrm{~m}^{2}$ & $14(48.3 \%)$ \\
\hline 3 - GFR $30-59 \mathrm{~mL} / \mathrm{min} / 1.73 \mathrm{~m}^{2}$ & $6(20.7 \%)$ \\
\hline 4 - GFR $15-29 \mathrm{~mL} / \mathrm{min} / 1.73 \mathrm{~m}^{2}$ & $0(0 \%)$ \\
\hline $5-$ GFR $<15 \mathrm{~mL} / \mathrm{min} / 1.73 \mathrm{~m}^{2}$ & $1(3.4 \%)$ \\
\hline
\end{tabular}


Table 1 (continued)

\begin{tabular}{|c|c|}
\hline Categories/grade - Description & $\begin{array}{l}\text { Mean } \pm \text { SD; } \\
\mathrm{N}(\%)\end{array}$ \\
\hline \multicolumn{2}{|l|}{ Hyperlipidemia } \\
\hline 0 - None & $7(24.1 \%)$ \\
\hline 1 - Elevated without drug treatment & $0(0 \%)$ \\
\hline 2 - Elevated with dietary treatment & $0(0 \%)$ \\
\hline 3 - Elevated with drug and diet treatment & $21(72.4 \%)$ \\
\hline 4 - Missing data & $1(3.4 \%)$ \\
\hline \multicolumn{2}{|l|}{ Cardiac status } \\
\hline 0 - Asymptomatic & $20(69 \%)$ \\
\hline 1 - Asymptomatic, Ml & $6(20.7 \%)$ \\
\hline 2 - Symptomatic, unstable angina, etc. & $3(10.3 \%)$ \\
\hline \multicolumn{2}{|l|}{ Pulmonary status } \\
\hline $0-$ Normal & $24(82.8 \%)$ \\
\hline 1 - Asymptomatic or mild dyspnea & $5(17.2 \%)$ \\
\hline 2 - Severe & $0(0 \%)$ \\
\hline \multicolumn{2}{|l|}{ Functional status } \\
\hline 0 - No impairment & $17(58.6 \%)$ \\
\hline 1 - Impaired, but able to carry out ADL without assistance & $4(13.8 \%)$ \\
\hline $\begin{array}{l}1 \text { - Needs some assistance to carry out ADL or ambulatory } \\
\text { assistance }\end{array}$ & $5(17.2 \%)$ \\
\hline 2 - Requiring total assistance for ADL or non-ambulatory & $3(10.3 \%)$ \\
\hline \multicolumn{2}{|l|}{ Rutherford } \\
\hline 4 & $16(55.2 \%)$ \\
\hline 5 & $10(34.5 \%)$ \\
\hline 6 & $3(10.3 \%)$ \\
\hline \multicolumn{2}{|l|}{ Fontaine } \\
\hline 3 & $16(55.2 \%)$ \\
\hline 4 & $13(44.8 \%)$ \\
\hline \multicolumn{2}{|l|}{ ASA } \\
\hline 1 & $0(0.0 \%)$ \\
\hline 2 & $5(17.2 \%)$ \\
\hline 3 & $21(72.4 \%)$ \\
\hline 4 & $3(10.3 \%)$ \\
\hline \multicolumn{2}{|l|}{ Wounds lower limbs } \\
\hline $0-\mathrm{No}$ & $16(55.2 \%)$ \\
\hline $1-$ Yes & $13(44.8 \%)$ \\
\hline \multicolumn{2}{|l|}{ Glass classification - Aorta-lliac } \\
\hline 0 & $10(34.5 \%)$ \\
\hline 1 & $11(37.9 \%)$ \\
\hline 2 & $7(24.1 \%)$ \\
\hline 3 & $1(3.4 \%)$ \\
\hline \multicolumn{2}{|l|}{ Glass classification - Femoro-popliteal-crural } \\
\hline 0 & $9(31.0 \%)$ \\
\hline 1 & $11(37.9 \%)$ \\
\hline 2 & $7(24.1 \%)$ \\
\hline 3 & $2(6.9 \%)$ \\
\hline \multicolumn{2}{|l|}{ Preoperative walking distance } \\
\hline 1 - None & $3(10.3 \%)$ \\
\hline $2-0-100 m$ & $14(48.3 \%)$ \\
\hline $3-101-500 m$ & $3(10.3 \%)$ \\
\hline $4-501-1000 m$ & $3(10.3 \%)$ \\
\hline $5->1000 m$ & $6(20.7 \%)$ \\
\hline Preoperative $\mathrm{ABI}(\mathrm{mmHg})$ & $53.7 \pm 32.0$ \\
\hline
\end{tabular}

- Only type-2 Diabetes Mellitus

Note: $\mathrm{BMI}=$ Body Mass Index. eGFR = estimated Glomerular Filtration Rate. ASA = American Society of Anesthesiologists. $\mathrm{ABI}=$ Ankle Brachial Index 


\subsubsection{Clinical parameters}

The clinical outcome parameters were: technical success of the revascularisation, Rutherford classification, global anatomic staging system (GLASS) classification, (time to) wound healing, wound ischemia and foot infection (WIFI) classification, $A B I$ and walking distance. Technical success of revascularisation was based on an in-house scoring system, constructed on peroperative digital subtraction angiography (DSA) images. This was scored, continuously, between 0 and $100 \%$ success guided by five categories (0\%: No revascularisation possible 25\%: unsatisfied (inflow up-to P3 insufficient) 50\%: average satisfied (inflow up-to P3 greatly improved) $75 \%$ : satisfied (good inflow including 1 patent crural artery) and 100\%: optimal result (complete inflow up to the foot arcade through a patent a. tibialis posterior and a. dorsalis pedis)). This scoring was performed independently and blinded for patient data by three vascular surgeons. From preoperative imaging (magnetic resonance imaging (MRI) or computed tomography (CT)) the location and severity of the stenosis/occlusion were collected and scored using the GLASS classification (12). Post-operative GLASS classification was determined on final DSA images. Improvement in Rutherford classification was determined at the final outpatient clinic visit before intervention and the first outpatient clinic visit within 30 days after intervention. $A B I$ and walking distance were measured at the final outpatient clinic visit before surgery and within 31 days after revascularisation. Time to wound healing was assessed during outpatient clinic visits. Clinical follow up was six months after the revascularisation procedure.

\subsubsection{Equipment}

A PeriCam PSI NR (Perimed AB, Stockholm, Sweden) LSCI system was used in this study. This LSCI system uses a divergent class 1 laser, with a wavelength of $785 \mathrm{~nm}$, to illuminate a tissue surface. A frame rate of 21 frames per second was used. These frames were averaged over 21 frames to suppress noise. A measurement distance of $30 \pm 10 \mathrm{~cm}$ was used to image both small and large feet. Data was analysed using the software tool Pimsoft (Perimed AB, Stockholm, Sweden). A C-arm (Discovery IGS740, GE Healthcare, Buc, France) was used to perform fluoroscopic guided revascularisation procedures. A handheld continuous wave ultrasound Doppler combined with a pressure cuff was used to determine ABI.

\subsubsection{Safety}

To safely introduce this LSCl system into the HOT, strict hospital guidelines for using laser systems in a HOT must be met. The used C-arm of the HOT determines its position in the operating room with a $785 \mathrm{~nm}$ wavelength laser. This wavelength is equal to the wavelength of the LSCl system. To avoid interference between the C-arm and $\mathrm{LSCl}$ system the LSCl system had to be shielded from the environment. An in-house designed box, made of black non-transparent Perspex, enveloping the foot was used to fully shield the LSCI laser from its surroundings. Furthermore, the LSCI system 
should not affect the sterility or patient safety in the HOT during the entire procedure. Placement of the LSCI system in the box did not influence laminar flow in the HOT, the box could be cleaned entirely with alcohol and was covered with sterile sheets during every procedure (Online Figure 1).

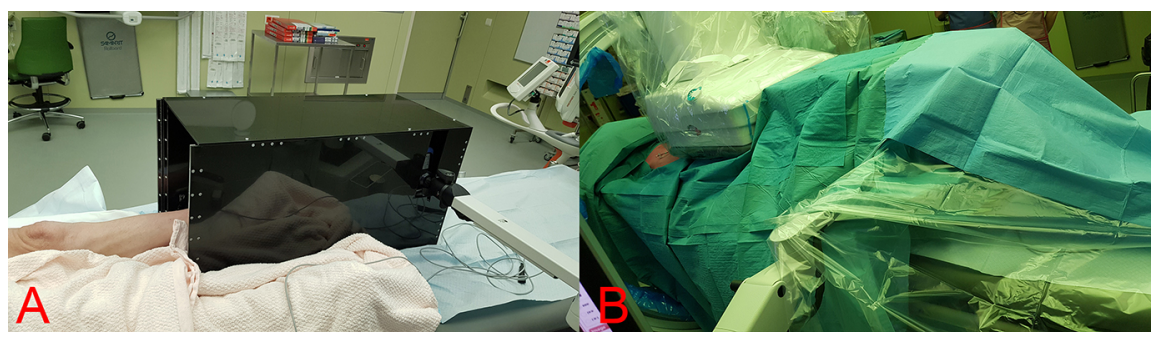

Online Figure 1: A: An in-house designed box, made of black non-transparent Perspex, enveloping the foot to shield the LSCI laser from its surroundings. B: Box covered with sterile sheets during the procedure.

\subsubsection{Measurement protocol}

After the positioning of the patient on the OR-table, the ipsilateral foot of the patient was placed in the box. After acclimatization to the OR temperature $\left(20^{\circ} \mathrm{C}\right)$, the $\mathrm{LSCl}$ measurement was started. After the preoperative surgical safety checklist (sign-in) procedure, general anesthesia was administered. Administration of general anesthesia was annotated in the measurement data. Each procedural event such as start of incision, insertion of the sheet, percutaneous transluminal angioplasty (PTA), stent placement, clamping or re-opening of vessels and administered medication was annotated. After the revascularisation procedure the LSCI measurement continued for an additional 10 minutes till the end of the anesthesia. During the procedure the vascular surgeon was blinded for the LSCI data.

\subsubsection{Data analysis}

$\mathrm{LSCl}$ data of each patient was postoperatively analysed by a single investigator (BW). A random sample of $20 \%$ of the $\mathrm{LSCl}$ data were analysed independently by a second investigator (OAM) to measure the inter-observer variation. For each measurement 12 different regions of interest $(\mathrm{ROI})$ were manually drawn to determine the mean perfusion in different areas of the foot. ROI 1 till 8 correspond with recognisable anatomical structures of the foot, while ROI 9 till 12 correspond with the angiosome concept (13). A final ROI, combining the ROI 9-12, was used for the analysis of the complete foot. This resulted in a total of 13 ROls that were used for perfusion analyses (Figure 1a). Besides the 13 ROls, three time periods of interest (TOls) were selected to analyse during different periods of the revisualisation procedure. TOI1 was from the start of the measurement until general anesthesia was administered, TOI2 was from general anesthesia administration until incision, and TOI3 was from the end of 
revascularisation until the patient awakened (Figure 1b). With these ROls and TOls the mean perfusion of different areas of the foot could be measured and compared during different stages of the revascularisation procedure (Figure 1c). The influence of general anesthesia on $\mathrm{LSCl}$ measurements was determined by calculating the difference between TOI1 and TOI2 (=TOI2-TOI1) (14). The difference between TOI1 and TOI3 (=TOI3-TOI1) shows the change between baseline perfusion without general anesthesia and the postoperative perfusion under general anesthesia. The difference between TOI2 and TOI3 (=TOI3-TOI2) was used to determine pre- and postrevascularisation $\mathrm{LSCl}$ differences during general anesthesia. Finally, changes in perfusion, between TOI1 - TOI3 and TOI2-TOI3, were related to changes in clinical parameters. Changes in clinical parameters were obtained from the pre- and postoperative values. When only the peroperative value was available, changes in perfusion were related to this single peroperative value.

A
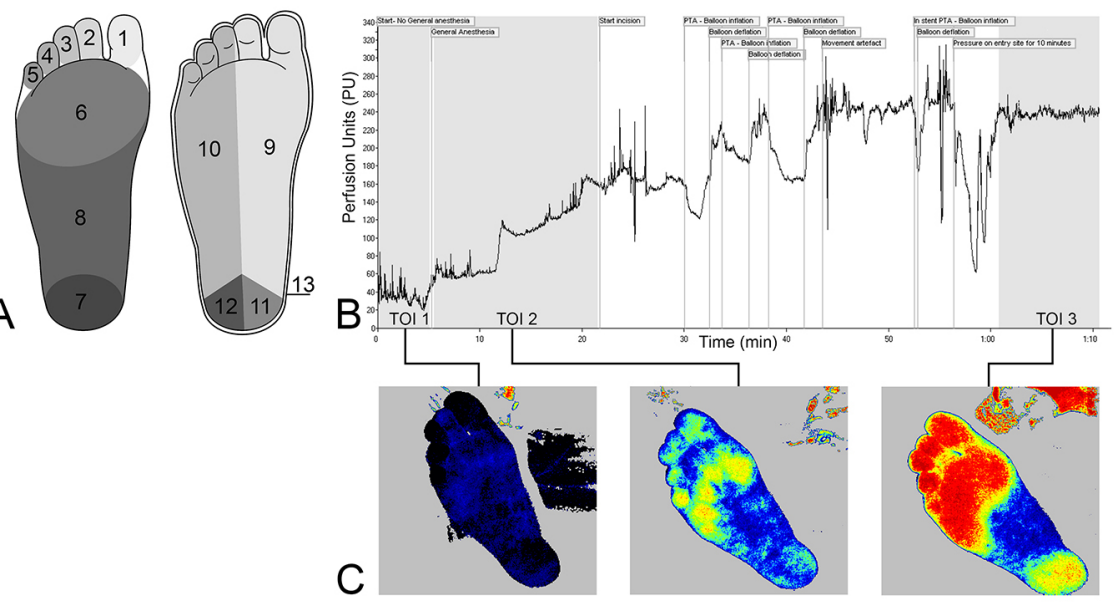

Figure 1:A: 13 Different Regions of Interest for each LSCI scan of the foot. B: Example of LSCI data of a complete revascularization. Annotations were placed to mark peroperative events. Moments of the TOls were visualized below the graph. C: Example of perfusion images for the three different TOIs.

\subsubsection{Statistical analysis}

Continuous variables were expressed, when normally distributed, as mean combined with the standard deviation (SD). Categorical variables were displayed as number with the corresponding percentage. Pre- and postoperative differences in continuous variables were analysed using the paired T-test, categorical variables were analysed using the $\mathrm{Chi}^{2}$-test. Inter observer variation was determined using the intraclass correlation coefficient (ICC) between the two observers for all 13 ROls. Within this study multiple data points were of interest, including the preoperative patient characteristics, the outcome of revascularisation treatment, change in $\mathrm{LSCl}$ perfusion 
values between TOI1-TOI3 and TOI2-TOI3 and postoperative outcome. These data points were analysed using a Pearson's correlation coefficient and a regression model. All statistical analyses were performed using IBM SPSS Statistics (Released 2016. IBM SPSS Statistics for Windows, Version 24.0. Armonk, NY: IBM Corp). For correlation coefficients values below 0.50 were scored as poor, between 0.50 and 0.75 as moderate, between 0.75 and 0.90 as good and above 0.90 as excellent (15). Measured $p$-values of less than 0.05 were regarded as statistically significant.

\subsection{RESULTS}

\subsubsection{Patient characteristics}

Data of one patient was not obtained, due to a preoperative descision to perform the procedure during local anaesthesia and was therefore excluded. An overview of the per- and post-operative patient characteristics of the remaining 29 patients with a complete LSCI dataset is shown in Table 2 .

Table 2: Per- and postoperative patient and treatment characteristics

\begin{tabular}{cl}
\hline Categories/grade - Description & Mean \pm SD \\
\hline Treatment - Location & (\%) \\
Aorta-iliac & 15 \\
AFC & 14 \\
Femoralis-P1 & 19 \\
P2-P3 & 10 \\
Crural & 8 \\
PTA (Single) & \\
PTA (Multiple) & $5(17.2 \%)$ \\
PTA + Stent & $4(13.8 \%)$ \\
PTA + Stent (Multiple) & $9(31.0 \%)$ \\
Treatment - Endovascular & $9(31.0 \%)$ \\
Endarterectomy & \\
Fem-Pop Bypass & $10(34.5 \%)$ \\
Fem-fem crossover & $1(3.4 \%)$ \\
Toe amputation & $1(3.4 \%)$ \\
Endarterectomy + toe Amputation & $2(6.9 \%)$ \\
Endarterectomy + Fem-fem crossover & $1(3.4 \%)$ \\
Treatment - open surgery & $2(6.9 \%)$ \\
1 & $71.9 \pm 20.4$ \\
Wrimary technical success & \\
Within 12 weeks & $4(30.8 \%)$ \\
Within 20 weeks & $9(69.2 \%)$ \\
Glass classification - Aorta-lliac & $11(84.6 \%)$ \\
Wealing weeks & $29(100.0 \%)$ \\
\hline & $0(0.0 \%)$ \\
\hline & $0(0.0 \%)$ \\
\hline & $0(0.0 \%)$ \\
\hline
\end{tabular}


Table 2 (continued)

\begin{tabular}{cl}
\hline Categories/grade - Description & Mean \pm SD \\
\hline Glass classification - Femoro-popliteal-crural & $\mathrm{N}(\%)$ \\
0 & $27(93.1 \%)$ \\
1 & $1(3.4 \%)$ \\
2 & $1(3.4 \%)$ \\
3 & $0(0.0 \%)$ \\
Walking distance & \\
$1-$ None & $5(17.2 \%)$ \\
$3-0-100 \mathrm{~m}$ & $3(10.3 \%)$ \\
$4-501-1000 \mathrm{~m}$ & $5(17.2 \%)$ \\
$5->1000 \mathrm{~m}$ & $2(6.9 \%)$ \\
Postoperative ABl (within 31 days) $(\mathrm{mmHg})$ & $13(44.8 \%)$ \\
\hline AB & $79.5 \pm 35.1$ \\
\hline
\end{tabular}

$\mathrm{ABI}=$ ankle brachial index.

\subsubsection{Inter observer variation}

There was no significant difference between the two observers and the ICC was good to excellent (ICC=0.825-1.000) (Figure 2).
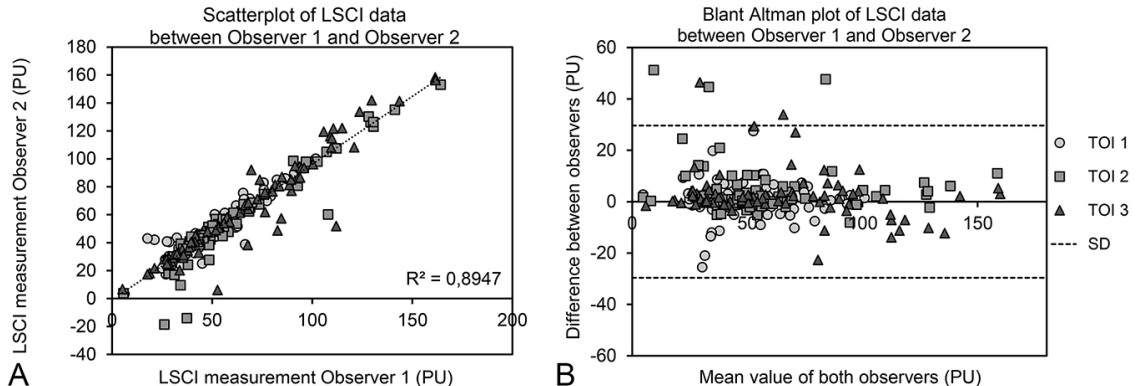

Figure 2: A: Scatterplot of $\mathrm{LSCl}$ data between different observers (BW and OM) for different TOls. B: Blant Altman plot of LSCI data between different observers (BW and OM) for different TOls.

\subsubsection{Clinical outcome parameters}

Technical success of treatment was $71.9 \pm 20.4 \%$ (Figure 3a). Rutherford classification was significantly improved after the revascularisation procedure $(4.5 \pm 0.5$ vs $2.2 \pm 2.3$, $p<0.001$ ) (Figure 3b). GLASS classification was significant improved after the revascularisation procedure (aortoiliac $0.97 \pm 0.87$ vs $0 \pm 0, p<0.001$; femoro-crural $1.07 \pm 0.92$ vs $0.1 \pm 0.41, p<0.001$ ), (Figure $3 c, d)$. There was a significant improvement in $A B I$ measured before and after the revascularisation procedure $(53.7 \pm 32.1 \mathrm{mmHg}$ vs $79.5 \pm 35.1 \mathrm{mmHg}, p=0.024$ ), (Figure $3 e$ ). Mean walking distance significantly improved after intervention $(1,7(<500 \mathrm{~m})$ vs $2.8(>500 \mathrm{~m}), \mathrm{p}=<0.001)$, (Figure $3 \mathrm{f})$. In total 13 patients suffered from wounds. Within the study period 8 patients showed full wound healing with a mean time to wound healing of $78 \pm 31$ days. In 4 patients the 
wound did not heal within 6 months, for one patient there was no information about wound healing. There was no significant difference in LSCI perfusion values between the patients with healed and non-healed wounds.
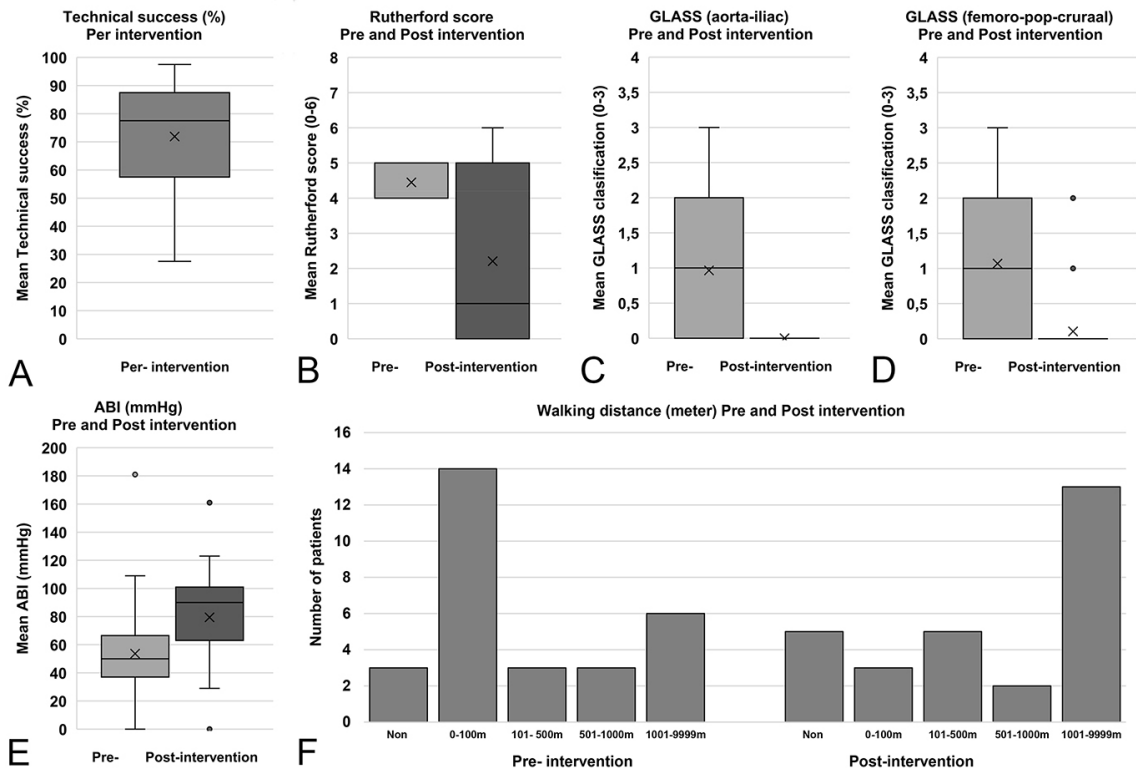

Figure 3: Boxplot visualisation of A: Technical success per intervention, B: Pre and post revascularisation Rutherford classification, C: Pre and post revascularisation GLASS aorta-iliac, D: Pre and post revascularisation GLASS aorta-iliac, E: Pre and post revascularisation ABI, F: Pre and post revascularisation walking distances.

\subsubsection{LSCl measurements}

Mean LSCI perfusion values for each ROI during each TOI are shown in Figure 4. The difference between TOls, per ROI, with the 95\% confidence interval are also shown. Mean LSCI perfusion values, for all ROls, measured during different TOls, increased during the intervention. The difference between TOI1 and TOI2 showed a significant increase in 8 of the 13 ROIs (ROI: 5-8, 10-13). A significant difference between TOI1 and TOI3, was found in 11 out of 13 ROIs (ROI: 2-3, 5-13). A significant difference between TOI2 and TOI3, where pre- and post-revascularisation perfusion differences were determined, was found in 3 of the 13 ROIs (ROI: $3,8,12$ ). 

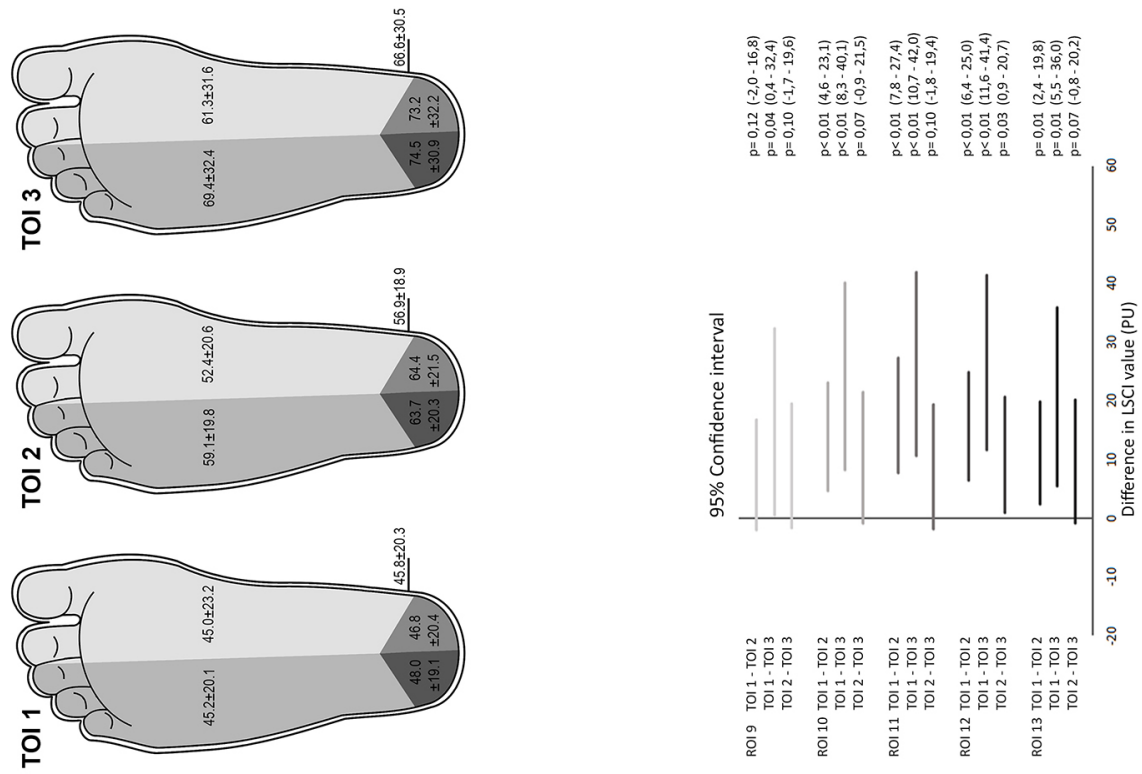

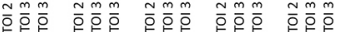

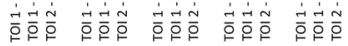

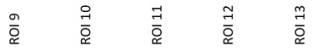
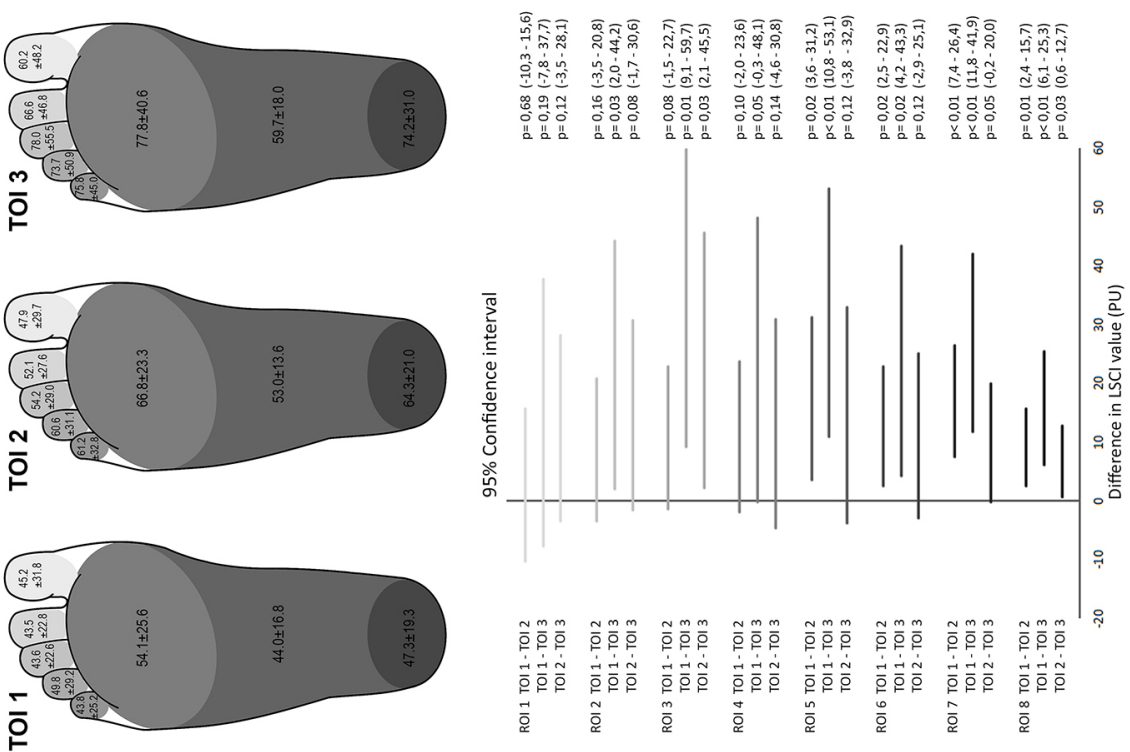

Figure 4: Mean LSCI values (PU) for all ROls combined with the 95\% confidence interval to show (significant) differences in PU between different TOls.

\subsubsection{Correlation $\mathrm{LSCl}$ and clinical prediction parameters}

The correlation between the differences in LSCI perfusion values (TOI3-TOI1 and TOI3-TOI2), of the entire plantar side of the foot (ROI13), and clinical parameters, were 
determined. Analysis showed a correlation coefficient of 0.23 between LSCI perfusion differences (TOI3-TOI1) and technical success, where a correlation coefficient of 0.28 was found between LSCI perfusion differences (TOI3-TOI2) and technical success (Figure $5 a$ and b).

For the difference between TOI1 and TOI3, both the $\mathrm{LSCl}$ perfusion values and Rutherford, $\mathrm{ABI}$ and walking distance increased or decreased in a corresponding manner in 25, 21 and 25 patients respectively. For the remaining 4, 8 and 4 patients contradicting outcomes were found, where LSCI perfusion values increased while the clinical parameter decreased or vice versa (Figure $5 \mathrm{c}$, i and k). For GLASS femorocrural, 9 patients had no disease, where 14 patients showed an improvement in GLASS with an improvement in LSCI perfusion values, 2 patients showed no improvement in GLASS while LSCI perfusion values in- or decreased. 4 patients showed an improvement in GLASS, while LSCI perfusion values decreased (Figure 5e). For GLASS aortoiliac, 10 patients had no disease, where 15 patients showed an improvement in GLASS with an improvement in LSCI perfusion values and 4 patients showed an improvement in GLASS, while LSCI perfusion values decreased (Figure $5 \mathrm{~g}$ ). Correlation analysis showed only non significant correlations coefficients of; 0.28 for Rutherford classification and LSCI, 0.03 for GLASS aortoiliac and LSCI, 0.13 for GLASS femoro-crural and LSCI and 0.33 for $\mathrm{ABI}$ and LSCI. For walking distance and $\mathrm{LSCl}$ a correlation coefficient of 0.48 was found. Furthermore, a correlation coefficient of 0.25 for wound healing and a correlation coefficient of $<0.01$ for time to wound healing was found.

For the difference between TOI2 and TOI3, both the $\mathrm{LSCl}$ perfusion values and Rutherford, $\mathrm{ABI}$ and walking distance increased or decreased in a corresponding manner in 25, 23 and 24 patients respectively. For the remaining 4, 6 and 5 patients contradicting results were found (Figure $5 \mathrm{~d}$, j and i). For GLASS femoro-crural, 14 patients showed an improvement in GLASS with an improvement in LSCI perfusion values 2 patients showed no improvement in GLASS while LSCI perfusion values in- or decreased. 4 patients showed an improvement in GLASS, while LSCI perfusion values decreased (Figure 5f). For GLASS aortoiliac, 16 patients showed an improvement in GLASS with an improvement in LSCI perfusion values, where 3 patients showed an improvement in GLASS, while LSCl perfusion values decreased (Figure 5h). Correlation analysis showed only non significant correlations coefficients of 0.36 for Rutherford classification and LSCI, 0.03 for GLASS aortoiliac and LSCI, 0.08 for GLASS femoro-crural and LSCI and of 0.45 for ABI and LSCI. For walking distance and $\mathrm{LSCl}$ a correlation coefficient of 0.44 was found. Furthermore, a correlation coefficient of 0.14 for wound healing and a correlation coefficient of 0.06 for time to wound healing was found. 


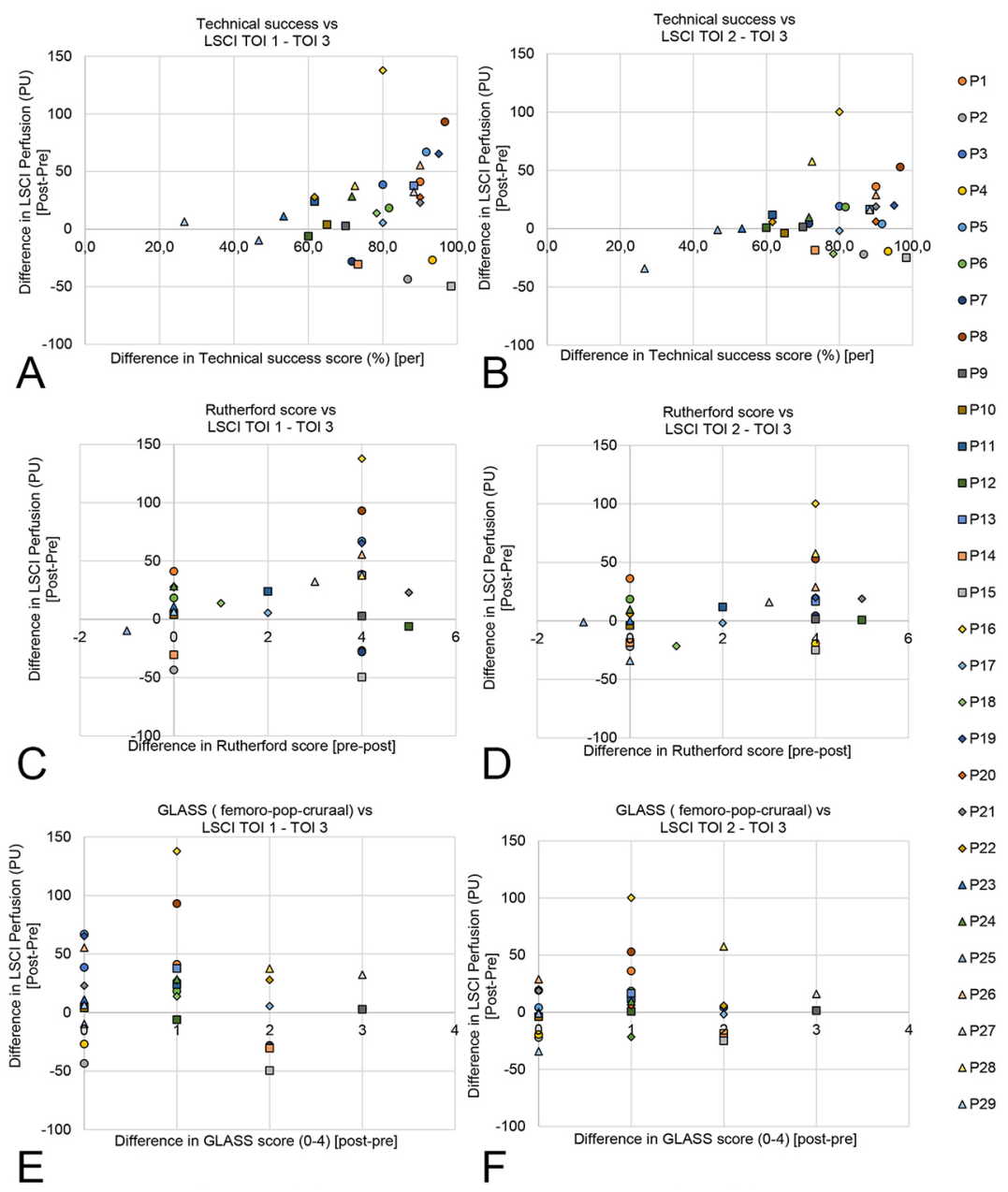

Figure 5: Visualisation of the difference in pre and post revascularisation clinical parameter versus the difference in LSCI perfusion values between TOI1-TOI3 and TOI2-TOI3. A: Technical success vs LSCI TOI1-TOI3, B: Technical success vs LSCI TOI2-TOI3, C: Rutherford classification vs LSCI TOI1-TOI3, D: Rutherford classification vs LSCI TOI2-TOI3, E: GLASS (femoro-pop-crural) vs LSCI TOI1-TOI3, F: GLASS (femoro-pop-crural) vs LSCI TOI2-TOI3 

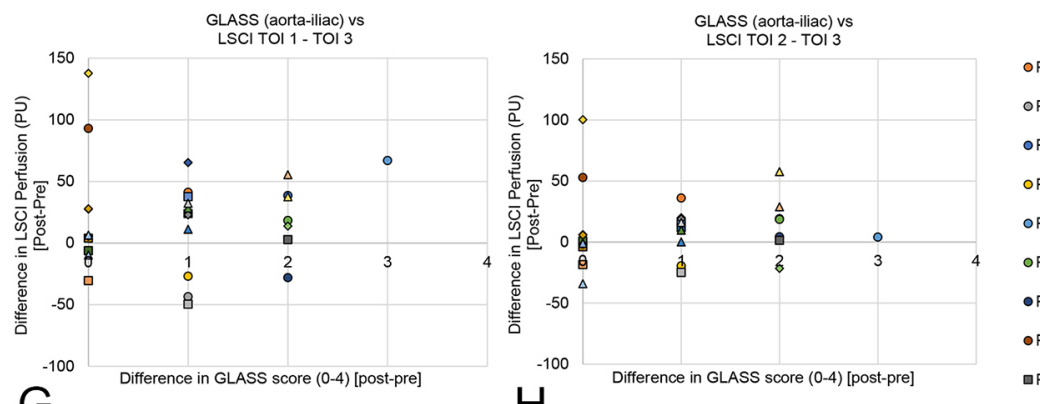

OP1

OP2

P3

OP4

OP5

oP6

คP7

๑P8

$\mathrm{H}$

$\square \mathrm{P9}$
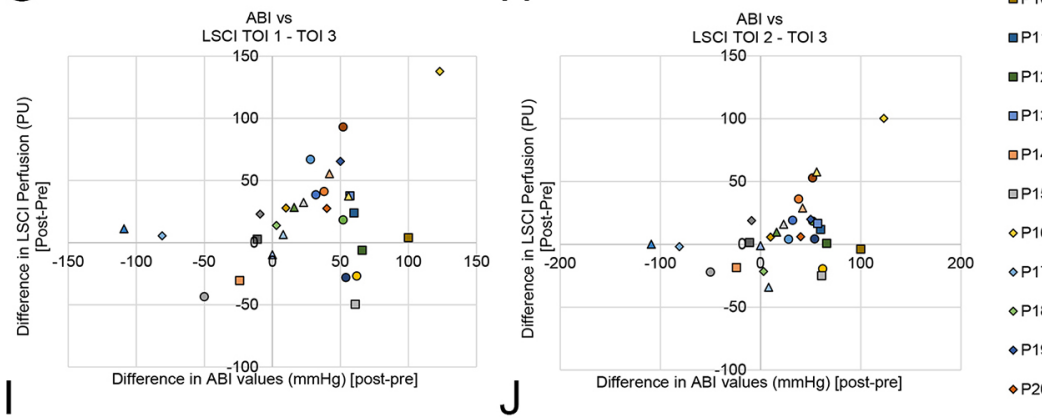

口P11

घP12

घP13

口P14

口P15

○P16

$\diamond \mathrm{P} 17$

$\diamond \mathrm{P} 18$

$\diamond$ P19<smiles>[AlH2]</smiles>

Walking distance vs

LSCI TOI 1 - TOI

$\mathrm{J}$
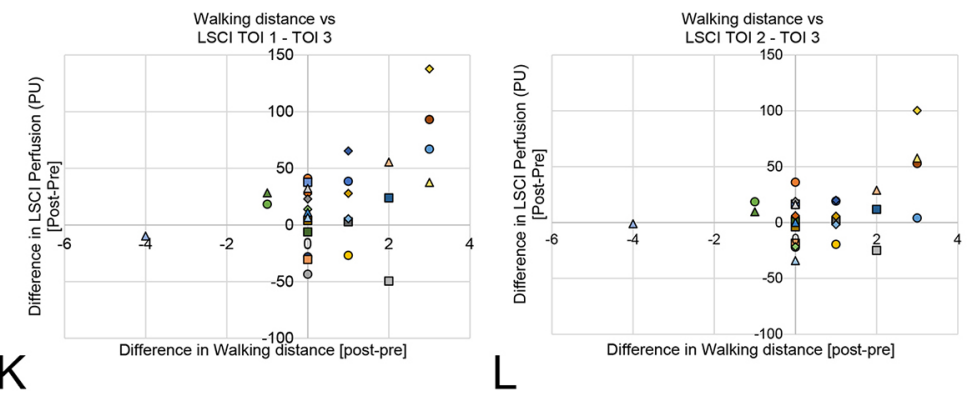

$\diamond$ P20

$\diamond \mathrm{P} 21$

$\diamond \mathrm{P} 22$

$\triangle \mathrm{P} 23$

$\triangle \mathrm{P} 24$

$\triangle \mathrm{P} 25$

$\triangle \mathrm{P} 26$

$\triangle \mathrm{P} 27$

$\triangle \mathrm{P} 28$

$\triangle P 29$

Figure 5 (continued): Visualisation of the difference in pre and post revascularisation clinical parameter versus the difference in LSCI perfusion values between TOI1-TOI3 and TOI2-TOI3. G: GLASS (aorta-iliac) vs LSCI TOI1-TOI3, H: GLASS (aorta-iliac) vs LSCI TOI2-TOI3, I: ABI vs LSCI TOI1-TOI3, J: ABI vs LSCI TOI2-TOI3, K: Walking distance vs LSCI TOI1-TOI3, L: Walking distance vs LSCI TOI2-TOI3. 


\subsubsection{Additional analysis of clinical parameters}

As shown in the Rutherford classification, $\mathrm{ABI}$ and walking distance graphs of figure 5 , three different groups of patients can be distinguished. The first group had an increase in both LSCI perfusion values and clinical parameters (group ++ ). The second group showed a decrease in both LSCI perfusion values and clinical parameters (group--). The third groups showed contradicting results (e.g. improved LSCI perfusion values with a decrease in clinical parameters or vice versa (group+- and group-+)). In order to determine what clinical parameter or patient characteristic can distinguish between the groups (group++ and group--), the groups were compared with each other. There was a significant difference between those groups in age, hypertension, cardiac status, Rutherford classification, Fontaine classification and presence of wounds as presented in Table 3.

Table 3: Clinical parameters or patient characteristics that can distinguish between the groups: group++ and group--

\begin{tabular}{|c|c|c|c|c|c|c|}
\hline \multirow{2}{*}{$\begin{array}{l}\text { Patient } \\
\text { Characteristics }\end{array}$} & \multicolumn{3}{|c|}{ TOI 1- TOI 3} & \multicolumn{3}{|c|}{ TOI 2 -TOI 3} \\
\hline & Group ++ & Group - - & $\mathrm{P}$-value & Group ++ & Group - - & $\mathrm{P}$-value \\
\hline Rutherford: & $\mathrm{n}=22$ & $\mathrm{n}=3$ & & $\mathrm{n}=22$ & $n=3$ & \\
\hline Age & 70.0 & 81.3 & $0.024^{*}$ & 69.2 & 79.0 & $0.016^{\star}$ \\
\hline Hypertension & $17 / 22$ & $3 / 3$ & $0.021^{*}$ & $17 / 22$ & $3 / 3$ & $0.021^{\star}$ \\
\hline Cardiac status & $13 / 22$ & $0 / 3$ & $<0.001^{*}$ & $13 / 22$ & $0 / 3$ & 0.599 \\
\hline Fontaine & 3.4 & 4.0 & $<0.001^{*}$ & 3.4 & 3.8 & 0.126 \\
\hline ABI: & $n=18$ & $n=3$ & & $\mathrm{n}=17$ & $n=5$ & \\
\hline Age & 68.3 & 81.3 & $0.019^{*}$ & 67.9 & 83.2 & $<0.001^{*}$ \\
\hline Hypertension & $14 / 18$ & $3 / 3$ & $0.042^{*}$ & $13 / 17$ & $5 / 5$ & $0.041^{*}$ \\
\hline Cardiac status & $10 / 18$ & $0 / 3$ & $<0.001^{*}$ & $9 / 17$ & $2 / 5$ & 0.654 \\
\hline Rutherford & 4.4 & 5.3 & 0.083 & 4.4 & 5.2 & $0.008^{*}$ \\
\hline Fontaine & 3.3 & 4.0 & $<0.001^{*}$ & 3.3 & 4.0 & $<0.001^{*}$ \\
\hline Wounds in limbs & $6 / 18$ & $3 / 3$ & 0.082 & $6 / 17$ & $5 / 5$ & $0.008^{*}$ \\
\hline Walking distance: & $\mathrm{n}=20$ & $\mathrm{n}=5$ & & $n=18$ & $n=6$ & \\
\hline Age & 70.6 & 79.0 & $0.026^{*}$ & 69.7 & 78.3 & $0.015^{\star}$ \\
\hline Hypertension & $15 / 20$ & $5 / 5$ & $0.021^{*}$ & $13 / 18$ & $6 / 6$ & $0.020^{*}$ \\
\hline
\end{tabular}

Note: ${ }^{\star} \mathrm{p}=<0.05$; ABI: ankle brachial index. TOl: time of interest. Group ++: increase in LSCI PU value and clinical outcome. Group - -: decrease in LSCI PU value and clinical outcome.

\subsection{DISCUSSION}

In this study we implemented LSCI peroperatively in a HOT to instantaneously measure the effect of the macrovascular revascularisation on the microcirculation. We related this with patient characteristics and short-term clinical outcome. The inter-observer variation of $\mathrm{LSCl}$ scans was good to excellent, meaning that LSCl-scan can be analysed by different observers without influencing results. The correlations between 
LSCI outcome and currently used clinical parameters for successful revascularisation were low.

Reproducibility and stability of LSCI were already analysed in a previous study that showed the influence of the frame rate, number of frames per image, movements of tissue, measuring distance and tissue curvature on the measured perfusion (16). This previous study showed unwanted influence of movement of tissue on measured perfusion. Frame rate and number of frames per image did not influence outcomes. Variations in measurement distance did not influence outcomes between 15 to $40 \mathrm{~cm}$ distance from the surface. Angle of incidence did not influence outcomes, when measurements were performed between $90 \pm 45^{\circ}$ in respect to the measured skin surface (16). Furthermore, another previous study supports $\mathrm{LSCl}$ as a stable and reproducible technique, within a population of patients with diabetic foot ulcers, during outpatient clinic measurements (10). All these factors combined with our findings gives us the confidence that $\mathrm{LSCl}$ can be used in a clinical setting by different observers. However, it should be noted that in our study both observers had experience in processing and analysing LSCl-scans and therefore the influence of a learning curve could not be determined. We expect that this learning curve will be very steep, and therefore non-experienced observers would learn quickly how to handle and interpret the LSCI measurements. Patient specific physiological parameters, like blood pressure or cardiac output could influence LSCl outcome values. This study did not relate these physiological parameters to peroperative $\mathrm{LSCl}$, which should be performed in a successive study.

The correlations between $\mathrm{LSCl}$ perfusion value differences and clinical parameters were low. However, a corresponding outcome was found in the majority of patients. When LSCl perfusion values increased, the technical success, Rutherford classification, GLASS classification, $\mathrm{ABI}$ and walking distance improved as well. When $\mathrm{LSCl}$ perfusion values decreased the technical success, Rutherford classification, ABI and walking distance deteriorated as well. Those corresponding outcomes are contradicting with the observed low correlation, but this discrepancy could be explained by multiple factors. First, after administering general anesthesia, the measured LSCI perfusion values increased. For ROI 5 to 8 and from 10 to 13 this increase was significant ( $p \leq 0.05)$. General anesthesia induces vasodilatation, resulting in a higher velocity and concentration of moving erythrocytes in the microcirculation (14). During general anesthesia, to level out the influence of this vasodilation effect, norepinephrine is used. This affects the perfusion in the microcirculation and most likely reduces the effect of the intervention on improvement of the microcirculation between the different TOIs. In this study, we did not consider the individual drug regulation of each patient and their corresponding effect on the microcirculation. Another option to determine the influence of general anesthesia and norepinephrine on a postoperative $\mathrm{LSCl}$ measurement is the inclusion of a fourth $\mathrm{TOI}$ one hour after the procedure. This 
measurement could be performed in the recovery room. Another explanation for the low to medium correlations could be the clinical and anatomical diversity of our patient population. Nevertheless, within this diversity we demonstrated that LSCI improvement has the potential to predict clinical outcome. However, in this study this wide variation resulted in small patient groups for each specific type/patient characteristic, making statistical analysis less powerful. We would recommend a follow up study with more patients (with the use of a power-calculation) to be able to prove possible statistically significant differences between the different stenosis/occlusion locations, interventions and clinical backgrounds. Furthermore, treatment of crural disease should be correlated with the angiosome concept, where revascularisation of one or more crural arteries could be correlated to the influence on perfusion of specific regions of the foot. A third possible reason why low correlation coefficients were found, are the used clinical endpoints. Rutherford classification, $A B I$ and walking distance can be determined at varying times before and after intervention. In some patients Rutherford classification, $\mathrm{ABI}$ and walking distance was determined up to 1 month before and/or after intervention. In that time these parameters could have changed and may not be representative for the direct pre- and post-intervention correlation. Unfortunately, direct determination of walking distance or $\mathrm{ABI}$, especially after crural revascularisation, is not possible or desirable.

We expect that implementation of a single imaging technique might not be sufficient to improve the revascularisation procedure. It sounds reasonable to combine different imaging techniques and use the combined prognostic value of those tests. A promising technique alongside LSCI to assess full lower limb circulation/perfusion/blood flow, is two-dimensional perfusion angiography (2D-PA), using standardised DSA images to determine blood flow up-to the microcirculation. By combining these techniques, a full perfusion status of the patients' foot could be assessed. Combining these techniques, improvement of in-and outflow up to the microcirculation could be determined, where $\mathrm{LSCl}$ gives information about this missing part of the puzzle, namely the improvement of the microcirculation.

Finally, when comparing the best scenario group (group ++ ) with the worst scenario group (group --) we found that; age, hypertension, cardiac status, Rutherford and Fontaine classification, $\mathrm{BMI}$ and presence of wounds were significant different between the groups. An in-depth analysis, in a larger population, should be performed to find the potential influence of those parameters on LSCl perfusion values during revascularisation. 


\subsection{CONCLUSION}

The high inter-observer agreement indicates that different observers can analyse LSCI data during revascularisation procedures without conflicting outcome, showing a high feasibility of LSCI. Significant improvement of LSCI perfusion values was found after revascularisation. Technical success was high and Rutherford classification, GLASS classification, $\mathrm{ABI}$ and walking distance significantly improved after intervention. A correlation between LSCI perfusion values and clinical outcome was poor. However, the majority of patients showed corresponding in- or decrease in both clinical outcome and LSCI perfusion values. 


\section{REFERENCES}

1. Becker F, Robert-Ebadi H, Ricco JB, Setacci C, Cao P, De Donato G, et al. Chapter I: Definitions, epidemiology, clinical presentation and prognosis. Eur J Vasc Endovasc Surg. 2011;42(SUPPL. 2):4-12.

2. Hirsch AT, Haskal ZJ, Hertzer NR, Bakal CW, Creager MA, Halperin JL, et al. ACC/AHA 2005 practice guidelines for the management of patients with peripheral arterial disease (lower extremity, renal, mesenteric, and abdominal aortic): Executive summary. Circulation. 2006;113(11):1474-547.

3. Ma KF, Kleiss SF, Schuurmann RCL, Bokkers RPH, Ünlü Ç, De Vries JPPM. A systematic review of diagnostic techniques to determine tissue perfusion in patients with peripheral arterial disease. Expert Rev Med Devices. 2019;16(8):697-710.

4. Norgren L, Hiatt WR, Dormandy JA, Nehler MR, Harris KA, Fowkes FGR, et al. InterSociety Consensus for the management of peripheral arterial disease (TASC II). J Vasc Surg. 2007;45(1):S5A-S67A.

5. Mennes OA, van Netten JJ, Slart RHJ., Steenbergen W. Novel Optical Techniques for Imaging Microcirculation in the Diabetic Foot. Curr Pharm Des. 2018 Mar 7;24(12):1304-16.

6. Eriksson S, Nilsson J, Sturesson C. Non-invasive imaging of microcirculation: A technology review. Med Devices Evid Res. 2014;7:445-52.

7. O'Doherty J, McNamara P, Clancy NT, Enfield JG, Leahy MJ. Comparison of instruments for investigation of microcirculatory blood flow and red blood cell concentration. J Biomed Opt. 2009;14(3):034025.

8. Humeau-Heurtier A, Abraham P, Henni S. Bi-dimensional variational mode decomposition of laser speckle contrast imaging data: A clinical approach to critical limb ischemia? Comput Biol Med. 2017;86:107-12.

9. Heeman W, Steenbergen W, van Dam GM, Boerma EC. Clinical applications of laser speckle contrast imaging: a review. J Biomed Opt. 2019 Aug 5;24(08):1.

10. Mennes OA, Van Netten JJ, Van Baal JG, Steenbergen W. Assessment of microcirculation in the diabetic foot with laser speckle contrast imaging. Physiol Meas. 2019;40(6):ab2058.

11. Stoner MC, Calligaro KD, Chaer RA, Dietzek AM, Farber A, Guzman RJ, et al. Reporting standards of the Society for Vascular Surgery for endovascular treatment of chronic lower extremity peripheral artery disease. J Vasc Surg. 2016;64(1):e1-21.

12. Conte MS, Bradbury AW, Kolh P, White J V, Dick F, Fitridge R, et al. Global vascular guidelines on the management of chronic limb-threatening ischemia. JVasc Surg. 2019 Jun;69(6S):3S-125S.e40.

13. van den Berg JC. Angiosome perfusion of the foot: An old theory or a new issue? Semin Vasc Surg. 2018 Jun;31(2-4):56-65.

14. Akata T, Warltier DC. General Anesthetics and Vascular Smooth Muscle. Anesthesiology. 2007 Feb;106(2):365-91.

15. Koo TK, Li MY. A Guideline of Selecting and Reporting Intraclass Correlation Coefficients for Reliability Research. J Chiropr Med. 2016;

16. Zötterman J, Mirdell R, Horsten S, Farnebo S, Tesselaar E. Methodological concerns with laser speckle contrast imaging in clinical evaluation of microcirculation. PLoS One. 2017;12(3):1-11. 
ASSESSING THE MICROCIRCULATION OF THE PLANTAR SIDE OF THE FOOT WITH LASER SPECKLE CONTRAST IMAGING DURING ENDOVASCULAR REVASCULARISATION PROCEDURES IN PATIENTS WITH PERIPHERAL ARTERIAL OCCLUSIVE DISEASE 



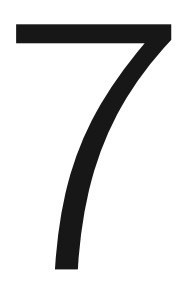

GENERAL DISCUSSION 
Chapter 7 
The overall aim of this thesis was to investigate the potential of novel optical techniques, and in particular the potential of Laser Speckle Contrast Imaging, for the assessment of microcirculation in diabetic foot disease and its applicability in clinical settings. In this final chapter, the research questions formulated in chapter 1 will be summarized, the limitations of research presented in this thesis will be discussed, and recommendations for future research and implications for clinical settings will be given.

\subsection{ANSWERS TO THE RESEARCH QUESTIONS}

1 What are the advantages and disadvantages of novel optical imaging techniques for local assessment of the microcirculation in diabetic foot ulcers?

The main results of our review presented in chapter 2 indicated that each of the four optical imaging techniques reviewed (Laser Speckle Contrast Imaging (LSCI), Laser Doppler Perfusion Imaging (LDPI), Photoacoustic Imaging (PAl) and Hyperspectral Imaging (HSI)) has its own advantages and disadvantages for imaging microcirculation in diabetic foot ulcers. These advantages and disadvantages depend on both the aims of measurements and the clinical relevance. Furthermore, we only found a limited number of publications in which clinically relevant outcomes for the assessment of microcirculation of diabetic foot ulcers were described. Therefore, one important recommendation for future research is to further examine the clinical applicability of these four techniques for diabetic foot ulcers in prospective trials.

In our review we particularly focused on the technical background, general clinical applications and the application for microcirculation assessment in diabetic foot ulcers of the four most common techniques. Regarding the technical background (the way how microcirculation can be captured) we found that LDPI and LSPI seem to be the most promising techniques when measuring blood flow or blood circulation. In contrast, PAl would be the best possible technique when the composition of tissue or the (relative) concentration of oxy- and deoxyhaemoglobin in blood should be measured. If the presence of blood should be measured, HSI or PAI would be the technique of choice. The different techniques also have different characteristics regarding 2D and $3 \mathrm{D}$ imaging. Whereas HSI would be well suited for 2D imaging in the plane of the skin surface, for 3D imaging PAI is better suited. With the deeper penetration depth of PAI it is possible to measure a $3 \mathrm{D}$ area in the foot.

We also considered aspects such as costs, usability and the need for non-contact methods. The most important outcomes regarding these aspects are that, in general, the costs for those novel optical imaging techniques are relatively high compared to the currently used non-invasive blood pressure imaging techniques. For example, the 
price of clinically available imaging techniques ranges between $€ 50.000$ and $€ 500.000$.

Furthermore, we found some differences in the usability of the four techniques. Although all four techniques are available in relatively mobile and compact systems (portable trolleys for bedside measurements for the patient), the usability for bedside measurements are different. For example, the majority of PAl-systems require a laser safety room. Furthermore, PAI needs a sound conducting medium (water/gel) between tissue and PAl-probe what makes it less useful for a preferably non-contact measurement.

2 What is the stability and reproducibility of $\mathrm{LSCl}$ for the assessment of microcirculation in diabetic foot ulcers and what is its relation to currently used non-invasive blood pressure measurement?

In the study presented in chapter 3 of this thesis, we found that LSCI is a stable and reproducible technique for the assessment of microcirculation in people with diabetic foot ulcers. Intra-observer agreements were high, inter-observer agreements were moderate to high and inter-assessor agreements were high. Also, we found high correlations between $\mathrm{LSCl}$ and currently used non-invasive blood pressure measurements (e.g. ankle pressure, toe pressure and transcutaneous oxygen pressure (TcpO2). LSCl further showed significant differences between non-ischemic, ischemic and critical-ischemic patient populations.

3 What is the prognostic value of LSCI for healing of diabetic foot ulcers?

In chapter 4 we continued with a prospective single centre cohort study and found no significant difference between patients with healed or non-healed foot ulcers for both the $\mathrm{LSCl}$ scans and blood pressure measurements at 12 and 26 weeks after measurement. Furthermore, the observed moderate sensitivity and specificity for both $\mathrm{LSCl}$ and non-invasive blood pressure measurements resulted in some small positive and negative likelihood ratios that indicate limited value of both types of measurements as prognostic instrument for foot ulcer healing. This could be an indication that the degree of perfusion does not seem to be predictive for the healing of diabetic foot ulcers in our measured patient population.

4 Can clinical applicability of LSCI be improved with a robust tracking algorithm for semi-automatic analysis of LSCI data?

The short answer to this research question is: yes. In chapter 5 we developed and validated a tracking algorithm for the analysis of $\mathrm{LSCl}$ data of diabetic foot ulcers. Results of the study showed that the algorithm has good to excellent inter-rater 
reliability when compared to the currently used standard assessment. The algorithm required minimal manual input and was able to process all $\mathrm{LSCl}$ data. The result was an approximately 10-fold workload reduction in comparison to the manual approach. Consequently, this tracking algorithm may have the potential to improve the clinical applicability of LSCI for the assessment of diabetic foot ulcers in the clinical setting.

5 Is LSCI clinically applicable during revascularization procedures and what is the potential clinical applicability of the LSCI parameters in patients with chronic limb threatening ischemia?

In the study described in chapter 6 , we found a good to excellent inter-observer agreement that indicates that different observers can analyse $\mathrm{LSCl}$ data during revascularization procedures with similar results. Furthermore, significant improvement was found between $\mathrm{LSCl}$ perfusion values measured before and after revascularization. However, the correlation between technical and clinical success of revascularization and $\mathrm{LSCl}$ scans was limited. We conclude that it is possible to use $\mathrm{LSCl}$ preoperatively to measure real-time blood flow, but the added value to the currently used clinical parameters seems low.

\subsection{DISCUSSION AND RECOMMENDATIONS}

This thesis demonstrated that LSCl adds some unique possibilities to the conventional non-invasive blood pressure measurements for the diabetic foot population. However, despite the possibilities, there are various limitations that have to be considered and recommendations for future research that may facilitate a successful implementation of $\mathrm{LSCl}$ in the clinic.

\subsubsection{Gold standard perfusion tests}

In this thesis, we aimed to validate LSCI measurements, by investigating whether LSCI is better or at least as good as other perfusion measurements. However, such a direct comparison was not possible, as there is no gold standard to measure perfusion available yet. Therefore, one has to think of other ways of comparing the results of the $\mathrm{LSCl}$ measurements. We compared LSCI data with currently used non-invasive blood pressure measurements. Alternative options are: phantom measurements or invasive diagnostic measurements.

As already mentioned, we compared our findings with non-invasive blood pressure measurements. With the calibration of the system, we did know that the measured perfusion in arbitrary Perfusion Units was stable over time and between patients. As such, we were able to measure and compare the LSCl outcomes with clinical data (e.g. 
non-invasive blood pressure measurements). It should be noted that non-invasive blood pressure measurements have limitations and therefore cannot be considered as gold standard for perfusion measurements. One of the limitations is that the currently used blood pressure measurements can be influenced by other factors $(1,2)$. Calcification in the vessels for example can cause stiffness in the blood vessels and this can result in incorrect blood pressure values. In addition, blood pressure does not provide a clear picture of the entire foot, but only of the extremities that are measured (ankle or toe). And finally, non-invasive blood pressure measurements measure the blood pressure in the macrocirculation, while we were interested in the perfusion measurement with LSCI in the microcirculation.

Alternatively, phantom measurements could be useful to objectively investigate the technical specifications of the LSCI device (Pericam PSI). In this thesis, we decided not to perform these measurements. We primarily wanted to focus on patient measurements because we wanted to focus on clinically relevant research. Also, we were already able to check the system's calibrations with a calibration box. The system was calibrated before its use by measuring a zero-perfusion area and a colloidal suspension of polystyrene particles to set the LSCI values on $0 \pm 5$ perfusion units (PU) and $250 \pm 5 \mathrm{PU}$ respectively. In this way we were able to calibrate the measured perfusion expressed in Perfusion Units (PU). Yet, we could not correlate those values in PU to perfusion expressed in SI units for flow such as $(\mathrm{ml} / \mathrm{g} / \mathrm{s})$ or pressure $(\mathrm{mmHg})$. Therefore, we were not able to compare perfusion measured with $\mathrm{LSCl}$ to other techniques that measure perfusion. In future research, it could be interesting to also perform these phantom measurements.

Furthermore, more advanced and invasive imaging techniques such as MRA, CTA or $\left[{ }^{15} \mathrm{O}\right]$-water PET might be used as gold standard because they can better visualize and quantify the blood flow in the entire foot. Yet, a disadvantage of these tests is that they are more invasive for the patient. For example, they need contrast agents, often they have longer measurement times and the waiting lists for participation are longer. Therefore, we decided not to include them in our research. Furthermore, although those test have a better visualization of blood present in the foot, it is still not a gold standard for perfusion measurements in the outer layer of the foot.

Because of those limitations and the lack of available gold standard measurements for perfusion, it was not surprising to find a moderate correlation between LSCI perfusion measurements and blood pressure measurements. This is an indication that LSCI does indeed measure something clinically different than what is measured with currently used blood pressure measurements. This results in the possibility that $\mathrm{LSCl}$ can overcome the disadvantages of the currently used non-invasive blood pressure measurements for the diagnosis, prognosis and treatment of diabetic foot ulcers. However, to fulfil this role, clinical cut-off values need to be distinguished. 


\subsubsection{Clinical cut-off values for diagnostic methods}

As mentioned above and in the introduction, the cut-off values for $\mathrm{LSCl}$ measurements were not yet known when we started our research. To further increase the clinical applicability of LSCI and its role in diagnosis, prognosis and treatment of diabetic foot ulcers, it would be important to determine those cut-off values. In the clinic, people work almost exclusively with standardized cut-off values that indicate a reduction in blood flow or have diagnostic or prognostic power. Not having these values makes it difficult to interpret and use LSCI data in a clinical setting.

For diagnostic purposes it was possible to find cut-off values that give an indication about the perfusion in the foot. This could help with the differentiation between nonischemic, ischemic and critical ischemic patients. In chapter 3 of this thesis, we investigated this diagnostic power of $\mathrm{LSCl}$ in a clinical setting and found significant differences between non-ischemic, ischemic and critical-ischemic patients. This study was relatively small and was performed in a single centre of expertise. Nevertheless, those results gave an indication that $\mathrm{LSCl}$ can be useful as a diagnostic tool and that clinical cut-off values for diagnostic purposes can be found. However, more research is needed to find and validate clear cut-off values. Furthermore, it should still be considered that those values might not exist at all. Instead of a single cut-off value, a range of values may be needed for a diagnosis. Furthermore, the possibility of diagnosing the level of ischemia in the diabetic foot is only the first step towards a successful implementation in the clinic.

It is also important to find the prognostic power of $\mathrm{LSCl}$. With the correct prognosis of a disease, the choice for adequate treatment can be improved. However, this thesis has shown that $\mathrm{LSCl}$ was less useful in the prognosis of outcomes of diabetic foot ulcers. As shown in chapters 4 and 6, the prognostic power of LSCI for wound healing or successful revascularization was limited. No significant correlation between LSCI and wound healing or successful revascularization was found. This was against our expectation since previous research did find an association between healing and noninvasive blood pressure values $(1,3)$. A reason for the contradicting results might be related to the cohorts investigated, since cohorts in other studies were mostly based on older cohort studies (1980-2018) (4). These older values may no longer be representative for the current patient population visiting diabetic foot expertise centres, because these older cohorts are based on patient populations in which both relatively healthy and complex patients were represented. In this group, the presence of ischemia or peripheral neuropathy and peripheral artery disease (PAD) are both major causes for poor healing outcomes (5-9). By measuring the blood flow or perfusion in an early stage, a distinction between the relatively healthy population with an ulcer and the more complex population with co-morbidities such as (critical)ischemia, PAD, calcifications or oedema was observed. This resulted in a stronger predictive power of 
the non-invasive blood pressure measurements than we measured for both LSCl and non-invasive blood pressure measurements in our studies. We hypothesize that the reason behind this is that the distribution of the current patient population has changed over the past years. The relatively healthy diabetic foot patients are mainly being treated by general practitioners or podiatrists nowadays and they do not necessarily visit a hospital. All the patients that are redirected to specialised care (e.g. hospital, wound expert centre, diabetic foot expert centre) show rather complex symptoms, which makes it difficult to clearly predict ulcer healing based on a single measurement. In our studies, we only included patients consulting specialised care due to logistic reasons. For future research, however, it is recommended to also include relatively healthy diabetic foot patients in a multi-centre study with centres in different levels of care.

Concluding, it is recommended to further look into the cut-off values or 'cut-off range'. Based on our previous argumentation, we would recommend that future research further investigates the cut-off values or range for LSCI. In chapters 3 and 4 of this thesis, we described how and in which range these values can be found. But to increase the generalizability of the values, this analysis should be performed in a larger population (1,000 - 10,000 patients), that includes both complex patients as well relatively healthy diabetic foot patients. We would recommend to also include patients that are currently being treated by general practitioners or podiatrists.

\subsubsection{Multivariate predicting model}

As already mentioned in the previous paragraph, both LSCI and non-invasive blood pressure measurements have limited prognostic power to predict wound healing. This is probably the result of the more complex nature of diabetic foot disease and the complex patient population that visits the hospital. However, even in a larger cohort, the predictive power of a single standalone measurement may still be limited.

The current population of people with a diabetic foot ulcer usually has multiple and complex comorbidities $(4,10,11)$. This would argue in favour of also measuring multiple criteria (e.g. registered data from the field of lifestyle and living situation (e.g. smoking/non-smoking, living alone or not, being able to check the feet on a daily basis or not)) and take those variables into account. In order to use all those measured parameters and criteria, a multivariate predicting model combining $\mathrm{LSCl}$ with multiple other variables could be of added value. The results of such a model may then lead to a better prognosis of ulcer outcomes. The specific contribution of $\mathrm{LSCl}$, as well as other non-invasive blood pressure measurements, can then be investigated from these model outcomes. Simultaneously, the combined prognostic power of different tests and variables included are likely to be an improvement to prognosis as done in current clinical practice. A multivariate analysis and a multivariate prediction model may help clinicians to better decide on the right treatment at the right time for the right patient. 
Therefore, we recommend to look further into the development of multivariate prediction models for ulcer outcomes, including $\mathrm{LSCl}$. While there is ongoing research into such predicting models $(12,13)$, such as the WiFI classification with its combination of wound extent, degree of ischemia, and extent of foot infection, more complex analysis are statistically and clinically possible. Including LSCI in these developments will provide further insights in the prognostic qualities of this non-invasive technique.

An addition to this recommendation would be to investigate the inclusion of repeated measurements of $\mathrm{LSCl}$ and similar techniques, throughout the treatment process of a diabetic foot ulcer. Combining microcirculatory information from multiple timepoints in a multivariate prediction model may reveal unknown prognostic characteristics of the technique.

\subsubsection{Outcomes to be predicted}

In our search for standard cut-off values for LSCI measurements, we focussed exclusively on values for the prognosis of ulcer healing. Although ulcer healing prediction is an important outcome for both patients and clinicians, it is not the only parameter that is useful in the clinical setting. Other outcomes can be predicted with LSCI, for which relevant cut-off values may be calculated. For example, lower-extremity amputation (major and/or minor) and foot infection, as outcomes directly associated with the ulcer, but also mortality, cardiovascular disease or quality of life may be chosen (4,14-16). A prognosis of an increased risk of amputation, infection or cardiovascular disease, or a patient likely to experience reduced quality of life in the near future may motivate clinicians and patients to intensify treatments.

It would be interesting to investigate if and how the prognostic power of $\mathrm{LSCl}$ would change if another clinical endpoint than ulcer healing would be investigated. For such endpoints, longer follow-up periods are needed than those used in our studies. Our chosen follow-up time of 6 months may be insufficient to discover all the long-term effects of a diabetic foot ulcer. For example, a chronic diabetic foot wound takes a long time to heal. Also, other comorbidities such as amputations, infections, mortality, cardiovascular disease or decreased in quality of life often manifest themselves after 6 months. We therefore recommend to add other clinical endpoints to a larger cohort study as described in the previous sections in order to investigate LSCI cut-off values and the prognostic power of a multivariate prediction model. Also we would recommend to extend the follow-up time to two years (17).

\subsubsection{Improving clinical utility of LSCI}

Using additional LSCI scans and measuring and analysing a multivariate prediction model as described in section 7.2.3, in combination with the usual tasks of a clinician can increase the workload and is therefore too complex for a clinical setting. Therefore, 
the use of $\mathrm{LSCl}$ and a multivariate prediction model in the clinic needs to be simplified. One possibility is the use of a tracking algorithm for the analysis of LSCl data. As presented in chapter 5 of this thesis, we developed and validated a semi-automatic algorithm. However, this algorithm still needs some manual input to perform the necessary calculations. Therefore, the medical practitioner is still needed for the analysis of the $\mathrm{LSCl}$ images, which can increase the chance of erroneous measurements and increases time required to obtain outcomes. A fully automated algorithm could be a solution to this problem. Such an algorithm should be able to detect the ulcer and the ulcer edge automatically, and subsequently analyse the data without the need of human input.

Another way of reducing the complexity is to combine different imaging techniques in one system in order to obtain multiple parameters in one measurement. For example, it may be possible to combine the used grey-scale images from the LSCI scans with full-colour images from a RGB-camera. This might be challenging because of the different positioning of both the RGB and LSCI cameras, resulting in slightly different viewing angles. But this could be solved by using a full colour calibration marker. Such a marker can help the algorithm to correct the different viewing angles. Furthermore it can compensate differences in colour because of lighting, shadows or measurement distance. Those full colour calibration markers (e.g. stickers or ink markings) should be positioned close to or on the foot before each scan in order to be easy to recognize and used during tracking.

Finally, it may also be possible to add both an infrared and a 3D camera to the system, in order to measure simultaneously the colour, temperature, and size (length, width and depth) of the ulcer. Those findings could then automatically be combined to measure the presence of an infection or to calculate different wound classifications (e.g. Texas Wound Classification or WiFl Classification). Ideally, all findings and measurements could then directly and automatically be combined in a multivariate prediction model that is integrated in the algorithm. This output could stimulate the use of LSCl data in a larger multi-centre study and hopefully improve the prognostic power of $\mathrm{LSCl}$ in the clinic.

\subsection{CONCLUDING REMARKS}

This thesis has demonstrated that LSCI is able to create clear, real-time perfusion images of microcirculation in the foot and ulcer of people with diabetes. LSCl can scan a complete foot in a non-invasive way and measure the perfusion real time in and around a foot ulcer. The system is reliable and stable, and has a low inter- and intraobserver variation. We also developed a tracking algorithm for the analysis of LSCI data 
that improves the use of $\mathrm{LSCl}$ in the clinical setting. Furthermore, $\mathrm{LSCl}$ shows both similarities and differences with the currently used non-invasive blood pressure measurements, which is an indication that it measures perfusion in a novel and different way than the currently used techniques. However, in our cohort we have not been able to link perfusion as measured with LSCI to clinical outcome parameters such as ulcer healing or successful revascularization. In other words, the technology as it is currently used has no added value in healthcare yet. Despite this current lack of applicability, this novel non-invasive optical imaging technique still offer potential to change clinical practice in the field of diabetic foot disease. For this, future research is needed to further investigate how LSCI can best be used to improve outcomes of diabetic foot ulcers. 


\section{REFERENCES}

1. Hinchliffe R, Brownrigg J, Apelqvist J, Boyko EJ, Fitridge R, Mills J, et al. IWGDF guidance on the diagnosis, prognosis and management of peripheral artery disease in patients with foot ulcers in diabetes. Diabetes Metab Res Rev. 2016;32:37-44.

2. Lipsky BA, Berendt AR, Cornia PB, Pile JC, Peters EJ AD. Infectious Diseases Society of America clinical practice guideline for the diagnosis and treatment of diabetic foot infections. Clin Infect Dis. 2012;54:e132-73.

3. Prompers L, Schaper N, Apelqvist J, Edmonds M, Jude E, Mauricio D, et al. Prediction of outcome in individuals with diabetic foot ulcers: Focus on the differences between individuals with and without peripheral arterial disease. The EURODIALE Study. Diabetologia. 2008:51(5):747-55.

4. Forsythe RO, Apelqvist J, Boyko EJ, Fitridge R, Hong JP, Katsanos K, et al. Performance of prognostic markers in the prediction of wound healing or amputation among patients with foot ulcers in diabetes: A systematic review. Diabetes Metab Res Rev . 2020 Mar 16;36(S1):e3278.

5. Holstein P, Lassen NA. Healing of ulcers on the feet correlated with distal blood pressure measurements in occlusive arterial disease. Acta Orthop . $1980 ; 51$ (16):995-1006.

6. Wallin L, Björnsson $\mathrm{H}$, Stenström A. Fluorescein angiography for predicting healing of foot ulcers. Acta Orthop . 1989; 60(1):40-4.

7. Tsai CY, Chu SY, Wen YW, Hsu LA, Chen CC, Peng SH, et al. The value of Doppler waveform analysis in predicting major lower extremity amputation among dialysis patients treated for diabetic foot ulcers. Diabetes Res Clin Pract. 2013 May;100(2):181-8.

8. Kalani M, Ostergren J, Brismar K, Jörneskog G, Fagrell B. Transcutaneous oxygen tension and toe blood pressure as predictors for outcome of diabetic foot ulcers. Diabetes Care . 1999 Jan; 22(1):147-51.

9. Faris I, Duncan H. Skin perfusion pressure in the prediction of healing in diabetic patients with ulcers or gangrene of the foot. J Vasc Surg . 1985 Jul 1; 2(4):536-40.

10. Lazzarini PA, Pacella RE, Armstrong DG, van Netten JJ. Diabetes-related lowerextremity complications are a leading cause of the global burden of disability. Diabet Med. 2018;

11. Fowler MJ. Microvascular and macrovascular complications of diabetes. Clin Diabetes. 2011;

12. aan de Stegge WB, Abu-Hanna A, Bus SA. Development of a multivariable prediction model for plantar foot ulcer recurrence in high-risk people with diabetes. BMJ Open Diabetes Res Care . 2020 Mar; 8(1):e001207. /pmc/articles/PMC7103819/

13. Monteiro-Soares M, Boyko EJ, Ribeiro J, Ribeiro I, Dinis-Ribeiro M. Predictive factors for diabetic foot ulceration: A systematic review. Vol. 28, Diabetes/Metabolism Research and Reviews. 2012. p. 574-600.

14. Alexiadou K, Doupis J. Management of diabetic foot ulcers. Diabetes Ther. 2012;3(1):1-15.

15. Prompers L, Schaper N, Apelqvist J, Edmonds M, Jude E, Mauricio D, et al. Prediction of outcome in individuals with diabetic foot ulcers: Focus on the differences between individuals with and without peripheral arterial disease. The EURODIALE Study. 
Diabetologia . 2008 May 23; 51(5):747-55.

16. Brownrigg JRWW, Hinchliffe RJ, Apelqvist J, Boyko EJ, Fitridge R, Mills JL, et al. Performance of prognostic markers in the prediction of wound healing or amputation among patients with foot ulcers in diabetes: A systematic review. Diabetes Metab Res Rev. 2016;32:128-35.

17. Jeffcoate WJ, Bus SA, Game FL, Hinchliffe RJ, Price PE, Schaper NC. Reporting standards of studies and papers on the prevention and management of foot ulcers in diabetes: required details and markers of good quality. Lancet Diabetes Endocrinol. 2016;4(9):781-8. 



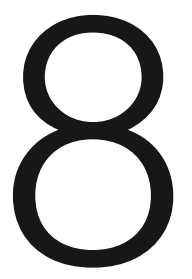

SUMMARY 
Chapter 8 
Diabetes Mellitus is one of the most common chronic disease worldwide and it has been estimated that the number of people with diabetes will grow because of our lifestyle changes and longer life-expectancy. This development is disturbing because diabetes has a severe impact on the patient's life. It can cause serious complications such as blindness, heart attacks, and strokes (1,2). Another severe and most frequently recognized complication of diabetes are diabetic foot ulcers which are defined as "foot ulcer in person with currently or previously diagnosed diabetes mellitus and usually accompanied by neuropathy and/or Peripheral Arterial Disease (PAD) in the lower extremity" (3). Diabetic foot ulcers are associated with high morbidity, mortality and healthcare costs (4-7). These consequences can even be more severe in case of diabetic foot ulcers with critical ischemia. Hence, an early and accurate diagnosis of this health condition is needed $(8,9)$. Today, the most common diagnosis of (critical-) ischemia is determined in clinical practice, using non-invasive measurements of blood flow in the feet, by means of assessments of the ankle pressure, toe pressure or transcutaneous oxygen pressure $(8,9)$. International guidelines provide the needed cut-off values for these measurements (8-10). Yet, these currently used non-invasive measurement techniques have various disadvantages. For example, they fail to adequately measure the microcirculation in the lower extremities at specific locations, and they can be influenced by factors such as oedema or infections $(8,11)$. Therefore, research into improved ways to assess the microcirculation in people with diabetic foot ulcers is needed. This thesis tried to fill this knowledge gap by looking into the potential of novel optical imaging techniques, and in particular in the potential of Laser Speckle Contrast Imaging (LSCl), for the assessment of the microcirculation in the diabetic foot and its applicability in the clinical setting. The research questions of this thesis were as follows:

1 What are the advantages and disadvantages of novel optical imaging techniques for local assessment of microcirculation in diabetic foot ulcers?

2 What is the stability and reproducibility of $\mathrm{LSCl}$ for the assessment of microcirculation in diabetic foot ulcers and what is its relation to currently used non-invasive blood pressure measurement?

3 What is the prognostic value of $\mathrm{LSCl}$ for healing of diabetic foot ulcers?

4 Can clinical applicability of LSCI be improved with a robust tracking algorithm for semi-automatic analysis of LSCI data?

5 Is LSCl clinically applicable during revascularization procedures and is there a correlation between LSCl outcomes to clinical parameters for the success of revascularization?

In chapter 2 of this thesis, the first research question was addressed. We performed a review of the following novel optical techniques for imaging microcirculation in the diabetic foot: Laser Doppler Perfusion Imaging (LDPI), Laser Speckle Contrast Imaging (LSCl), Photoacoustic Imaging (PAI), and Hyperspectral Imaging (HSI). For each 
technique, we had a closer look at its technical background, general clinical applications and its application for microcirculation assessment in diabetic foot ulcers. We found that each technique has specific advantages and disadvantages for imaging microcirculation and consequently, the clinical use should be determined based on measurement aims, and clinical relevance. Another important outcome of this review was that only a small number of publications describing clinical outcomes could be found, meaning that none of the four techniques has a strongly established clinical relevance yet.

The chapters 3 to 6 particularly focused on LSCI. Previous research has shown that LSCI can successfully be applied in the assessment of burns and burn-related wounds (12). Also, two other studies showed the potential of using LSCI in the diabetes context $(13,14)$. They found that LSCI could be used for diagnostic screening or monitoring of the capillary blood flow on a regular basis during treatment. Yet, they did not examine whether $\mathrm{LSCl}$ can reliably be used in people with diabetes. Therefore, in chapter 3 , we conducted a prospective, single centre, observational cohort study, investigating the stability and reproducibility of $\mathrm{LSCl}$ for the assessment of microcirculation in the diabetic foot (research question 2). We found that $\mathrm{LSCl}$ is a suitable method to assess microcirculation in people with diabetic foot ulcers since it was proven to be a stable technique with a high inter- and intra-user reliability. Also, results have shown that LSCI can distinguish between non-ischemic, ischemic and critical-ischemic patient populations.

For a successful implementation in the clinical setting, it is important to know what the clinical benefit of this LSCI technique is. Therefore, insight in its prognostic accuracy in comparison to non-invasive blood pressure measurements is required. Consequently, chapter 4 continued with a prospective single centre cohort study that was conducted to investigate the prognostic values of $\mathrm{LSCl}$ compared to non-invasive blood pressure measurements in relation to healing of diabetic foot ulcers. Results of this study show that there is no association between ulcer healing at 12 and 26 weeks after measurement and LSCl- measured microcirculation or non-invasive blood pressure measurements, indicating that the degree of perfusion does not seem to be predictive for the healing of diabetic foot ulcers. We can conclude that both types of measurements were not useful as a standalone prognostic instrument for diabetic foot ulcer healing.

A benefit of $\mathrm{LSCl}$ is that it can be used to measure different regions of interest (ROIs) of the diabetic foot ulcers simultaneously. For example, both the ulcer and the ulcer edge can be assessed and also multiple ulcers on one foot can be analysed (15). However, the ROls have to be drawn manually, which is rather time consuming and therefore limits the applicability of this technique in the clinical setting. An algorithm that assists in positioning the ROls could help to overcome this problem. In chapter 5 , 
we therefore continued with the development and validation of a fast and robust tracking algorithm for semi-automatic analysis of LSCI data, to improve applicability of $\mathrm{LSCl}$ in the clinical setting (research question 4). We succeeded in developing a tracking algorithm that shows a 10-fold workload reduction compared to the labourintensive manual approach.

In the final study described in chapter 6 , we investigated the clinical applicability of $\mathrm{LSCl}$ to assess the microcirculation during revascularization procedures in patients with chronic limb threatening ischemia $(\mathrm{CLTI})$. A prospective, exploratory, single centre cohort study was conducted to answer the fifth research question of this thesis: "Is LSCI clinically applicable during revascularization procedures and is there a correlation between LSCl outcomes to clinical parameters for the success of revascularization?". The results demonstrated a good to excellent inter-observer variation, indicating that different observers are capable to analyse LSCI data without conflicting interpretations. The correlation between LSCI outcome parameters and currently used clinical parameters for successful revascularization were poor.

In conclusion, chapter 7 summarizes the main findings of the five research questions of this thesis. Also, the limitations of the work in the thesis were discussed and recommendations for future research and implications for clinical practice were given. $\mathrm{LSCl}$ is able to create clear, real-time perfusion images of microcirculation in the foot and ulcer of people with diabetes. LSCI can scan a complete foot in a non-invasive way and measure the perfusion real time in and around a foot ulcer. The system is reliable and stable, and has a low inter- and intra-observer variation. We also developed a tracking algorithm for the analysis of LSCl data that improves its use in the clinical setting. Furthermore, LSCl shows both similarities and differences with the currently used non-invasive blood pressure measurements, which is an indication that it measures perfusion in a novel and different way than the currently used techniques. However, in our cohort we have not been able to link perfusion as measured with LSCI to clinical outcome parameters such as ulcer healing or successful revascularization. In other words, the technology as it is currently used has no added value in healthcare yet. Despite this current lack of applicability, this novel non-invasive optical imaging technique still offer potential to change clinical practice in the field of diabetic foot disease. For this, future research is needed to further investigate how $\mathrm{LSCl}$ can best be used to improve outcomes of diabetic foot ulcers. 


\section{REFERENCES}

1. IDF Diabetes Atlas 9th edition 2019 Available online: https://www.diabetesatlas.org/en/ (accessed on Feb 7, 2021).

2. Lazzarini, P.A.; Pacella, R.E.; Armstrong, D.G.; van Netten, J.J. Diabetes-related lowerextremity complications are a leading cause of the global burden of disability. Diabet. Med. 2018,

3. van Netten, J.J.; Bus, S.A.; Apelqvist, J.; Lipsky, B.A.; Hinchliffe, R.J.; Game, F.; Rayman, G.; Lazzarini, P.A.; Forsythe, R.O.; Peters, E.J.; et al. Definitions and criteria for diabetic foot disease on behalf of the International Working Group on the Diabetic Foot. 2020, 19.

4. Alexiadou, K.; Doupis, J. Management of diabetic foot ulcers. Diabetes Ther. 2012, 3, $1-15$.

5. Kerr, M.; Rayman, G.; Jeffcoate, W.J. Cost of diabetic foot disease to the National Health Service in England. Diabet. Med. 2014, 31, 1498-1504.

6. Prompers, L.; Huijberts, M.; Schaper, N.; Apelqvist, J.; Bakker, K.; Edmonds, M.; Holstein, P.; Jude, E.; Jirkovska, A.; Mauricio, D.; et al. Resource utilisation and costs associated with the treatment of diabetic foot ulcers. Prospective data from the Eurodiale Study. Diabetologia 2008, 51, 1826-1834.

7. Prompers, L.; Schaper, N.; Apelqvist, J.; Edmonds, M.; Jude, E.; Mauricio, D.; Uccioli, L.; Urbancic, V.; Bakker, K.; Holstein, P.; et al. Prediction of outcome in individuals with diabetic foot ulcers: Focus on the differences between individuals with and without peripheral arterial disease. The EURODIALE Study. Diabetologia 2008, 51, 747-755,

8. Hinchliffe, R.; Brownrigg, J.; Apelqvist, J.; Boyko, E.J.; Fitridge, R.; Mills, J.; Reekers, J.; Shearman, C.; Zierler, R.; Schaper, N.; et al. IWGDF guidance on the diagnosis, prognosis and management of peripheral artery disease in patients with foot ulcers in diabetes. Diabetes. Metab. Res. Rev. 2016, 32, 37-44.

9. Hinchliffe, R.J.; Forsythe, R.O.; Apelqvist, J.; Boyko, E.J.; Fitridge, R.; Hong, J.P.; Katsanos, K.; Mills, J.L.; Nikol, S.; Reekers, J.; et al. Guidelines on diagnosis, prognosis, and management of peripheral artery disease in patients with foot ulcers and diabetes (IWGDF 2019 update). Diabetes. Metab. Res. Rev. 2020, 36,.

10. Forsythe, R.O.; Apelqvist, J.; Boyko, E.J.; Fitridge, R.; Hong, J.P.; Katsanos, K.; Mills, J.L.; Nikol, S.; Reekers, J.; Venermo, M.; et al. Performance of prognostic markers in the prediction of wound healing or amputation among patients with foot ulcers in diabetes: A systematic review. Diabetes. Metab. Res. Rev. 2020, 36, e3278.

11. Lipsky, B.A.; Aragón-Sánchez, J.; Diggle, M.; Embil, J.; Kono, S.; Lavery, L.; Senneville, É.; Urbančič-Rovan, V.; Van Asten, S. IWGDF guidance on the diagnosis and management of foot infections in persons with diabetes. Diabetes. Metab. Res. Rev. 2016, 32, 45-74.

12. Ganapathy, P.; Tamminedi, T.; Qin, Y.; Nanney, L.; Cardwell, N.; Pollins, A.; Sexton, K.; Yadegar, J. Dual-imaging system for burn depth diagnosis. Burns 2014, 40, 67-81.

13. de M. Matheus, A.S.; Clemente, E.L.S.; de Lourdes Guimarães Rodrigues, M.; Torres Valença, D.C.; Gomes, M.B. Assessment of microvascular endothelial function in type 1 diabetes using laser speckle contrast imaging. J. Diabetes Complications 2017, 31 , 753-757.

14. Jayanthy, A.K.; Sujatha, N.; Reddy, M.R.; Narayanamoorthy, V.B. Non invasive blood flow assessment in diabetic foot ulcer using laser speckle contrast imaging technique. 
In Proceedings of the Arch Ophthalmol; Wax, A., Backman, V., Eds.; International Society for Optics and Photonics, 2014; Vol. 8952, p. 89521D.

15. Mennes, O.A.; van Netten, J.J.; Slart, R.H.J..; Steenbergen, W. Novel Optical Techniques for Imaging Microcirculation in the Diabetic Foot. Curr. Pharm. Des. 2018, 24, 1304-1316. 



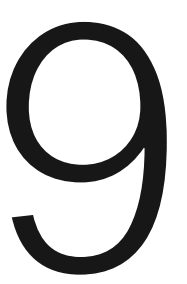

SAMENVATTING 
Chapter 9 
Diabetes mellitus (suikerziekte) is een van de meest voorkomende chronische ziekten wereldwijd en het aantal mensen met diabetes zal naar schatting toenemen. Dit heeft te maken met veranderingen in leefstijl (zoals overgewicht en obesitas, en fysieke inactiviteit) en een langere levensverwachting. Deze ontwikkeling is verontrustend omdat diabetes een ernstige invloed heeft op het leven van de patiënt. Diabetes kan ernstige complicaties veroorzaken, zoals blindheid, hartaanvallen en beroertes $(1,2)$. Een andere ernstige en meest voorkomende complicatie van diabetes zijn diabetische voetulcera, die worden gedefinieerd als; voetulcus bij een persoon met momenteel of eerder gediagnosticeerde diabetes mellitus en die gewoonlijk gepaard gaat met neuropathie en / of perifeer vaatlijden (PAD) in de onderste extremiteit (3). Diabetische voetulcera worden geassocieerd met hoge morbiditeit (ziektecijfer), mortaliteit (sterftecijfer) en zorgkosten (4-7). Deze gevolgen kunnen nog ernstiger zijn als er sprake is van diabetische voetulcera met kritische ischemie (verminderde weefseldoorbloeding). Een vroege en nauwkeurige diagnose van deze gezondheidstoestand is daarom van groot belang $(8,9)$. Tegenwoordig wordt (kritische-) ischemie in de klinische praktijk vooral gediagnosticeerd met behulp van niet-invasieve bloeddrukmetingen in de voeten. Dit wordt gedaan door middel van metingen van de enkeldruk, teendruk of transcutane zuurstofspanning $(8,9)$. Internationale richtlijnen geven de nodige grenswaarden voor deze metingen (8-10). Echter hebben deze niet-invasieve meetmethoden een aantal nadelen. Zo kunnen deze de microcirculatie in de onderste ledematen op specifieke locaties niet adequaat meten. Daarnaast kunnen niet-invasieve meetmethoden worden beïnvloed door factoren als oedemen (vochtophopingen) of infecties $(8,11)$. Daarom is het belangrijk dat er onderzoek wordt uitgevoerd naar verbeterde manieren om de microcirculatie bij mensen met diabetische voetulcera in kaart te brengen. Dit proefschrift probeerde deze kenniskloof te dichten door de mogelijkheden van nieuwe optische beeldvormingstechnieken te verkennen. Specifiek zijn de mogelijkheden van Laser Speckle Contrast Imaging (LSCI) onderzocht, zowel voor de beoordeling van de microcirculatie in de diabetische voet, als de mate waarin deze techniek toepast kan worden in de klinische praktijk. De onderzoeksvragen van dit proefschrift waren als volgt:

1 Wat zijn de voor- en nadelen van nieuwe optische beeldvormingstechnieken voor de lokale beoordeling van de microcirculatie bij diabetische voetulcera?

2 Wat is de stabiliteit en reproduceerbaarheid van LSCI voor de beoordeling van microcirculatie in de diabetische voet en wat is de relatie met de momenteel gebruikte niet-invasieve bloeddrukmetingen?

3 Wat is de prognostische waarde van LSCI voor de genezing van diabetische voetulcera?

4 Kan de klinische toepasbaarheid van LSCI worden verbeterd met een algoritme voor semi-automatische analyses van LSCl-gegevens? 
5 Is LSCI klinisch toepasbaar tijdens revascularisatie procedures en is er een relatie tussen LSCl-uitkomsten en klinische parameters voor het succes van revascularisatie?

In hoofdstuk 2 van dit proefschrift werd de eerste onderzoeksvraag beantwoord. We hebben een literatuuronderzoek uitgevoerd naar nieuwe optische technieken voor het afbeelden van microcirculatie in de diabetische voet: Laser Doppler Perfusion Imaging (LDPI), Laser Speckle Contrast Imaging (LSCl), Photoacoustic Imaging (PAI) en Hyperspectral Imaging (HSI). Voor elke techniek hebben we de technische achtergrond, algemene klinische toepassingen en de toepassing voor de beoordeling van de microcirculatie bij diabetische voetulcera in kaart gebracht. Hieruit kwam naar voren dat elke techniek specifieke voor- en nadelen heeft voor het meten van microcirculatie. Een keuze voor een bepaalde techniek kan dus het beste gemaakt worden op basis van de doelstellingen en klinische relevantie. Een andere belangrijke uitkomst van dit literatuuronderzoek was dat er slechts een beperkt aantal wetenschappelijke publicaties gevonden kon worden waarin klinische uitkomsten werden beschreven. Dit betekent dat voor geen van de vier technieken een sterke klinische relevantie vastgesteld is tot nu toe.

In de hoofdstukken 3 tot en met 6 is de focus specifiek op LSCI gericht. Eerder onderzoek heeft aangetoond dat LSCl met succes kan worden toegepast bij de beoordeling van brandwonden (12). Ook hebben twee andere onderzoeken laten zien dat LSCl succesvol toegepast kan worden in de diabetescontext $(13,14)$. Er is gevonden dat LSCI gebruikt kan worden voor diagnostische screening of het regelmatig controleren van de capillaire bloedstroom tijdens de behandeling. Echter is er niet onderzocht of LSCI betrouwbaar kan worden gebruikt bij mensen met diabetes. Daarom hebben we in hoofdstuk 3 een prospectieve, single-center, observationele cohortstudie uitgevoerd, waarin we de stabiliteit en reproduceerbaarheid van LSCI hebben onderzocht voor de beoordeling van microcirculatie in de diabetische voet (onderzoeksvraag 2). Uit de resultaten kwam naar voren dat LSCI een stabiele techniek is met een hoge inter- en intra-beoordelaarsbetrouwbaarheid. Deze methode lijkt op basis daarvan geschikt te zijn om de microcirculatie bij mensen met diabetische voetulcera in kaart te brengen. De resultaten lieten ook zien dat LSCI niet-ischemische, ischemische en kritisch-ischemische patiënten populaties kan onderscheiden.

Voor een succesvolle implementatie in de klinische praktijk is het belangrijk om te weten wat het klinische voordeel is van deze LSCI-techniek. Daarvoor is inzicht nodig in de prognostische nauwkeurigheid van deze techniek, in vergelijking met nietinvasieve bloeddrukmetingen. In hoofdstuk 4 zijn we daarom verder gegaan met een prospectieve single-center cohortstudie. Doel van dit onderzoek was om de prognostische waarden van LSCI te onderzoeken in vergelijking met niet-invasieve bloeddrukmetingen in relatie tot genezing van diabetische voetulcera. Resultaten van 
deze studie tonen aan dat er geen associatie is tussen de genezing van ulcera 12 en 26 weken na de meting en LSCl-gemeten microcirculatie of niet-invasieve bloeddrukmetingen. Dit geeft aan dat de mate van perfusie niet voorspellend lijkt te zijn voor de genezing van diabetische voetulcera. We kunnen concluderen dat beide soorten metingen niet bruikbaar waren als op zichzelf staand prognostisch meetsysteem voor de genezing van diabetische voetulcera.

Een voordeel van $\mathrm{LSCl}$ is dat het kan worden gebruikt om verschillende interessegebieden (ROI's) van diabetische voetulcera tegelijkertijd te meten. Zo kunnen zowel de wond als de rand van de wond beoordeeld worden en kunnen ook meerdere wonden op één voet worden geanalyseerd (15). De ROl's moeten echter handmatig worden gepositioneerd, wat nogal tijdrovend is. Hierdoor is de toepasbaarheid van deze techniek in de klinische praktijk beperkt. Een algoritme dat helpt bij het positioneren van de ROl's, zou dit probleem kunnen oplossen. In hoofdstuk 5 gingen we daarom verder met de ontwikkeling en validatie van een snel en robuust tracking-algoritme voor semiautomatische analyse van LSCl-data. Het doel hiervan was om de toepasbaarheid van LSCI in de klinische praktijk te verbeteren (onderzoeksvraag 4). We zijn erin geslaagd een tracking-algoritme te ontwikkelen dat een 10-voudige vermindering van de werklast laat zien in vergelijking met de arbeidsintensieve handmatige aanpak.

In de laatste studie die beschreven is in hoofdstuk 6, onderzochten we de klinische toepasbaarheid van LSCl om de microcirculatie tijdens revascularisatie procedures bij patiënten met chronische ledemaatbedreigende ischemie (CLTI) te beoordelen. Een prospectieve, verkennende, single-center cohortstudie werd uitgevoerd om de vijfde onderzoeksvraag van dit proefschrift te beantwoorden: "Is LSCI klinisch toepasbaar tijdens revascularisatie procedures en is er een relatie tussen LSCl-uitkomsten en klinische parameters voor het succes van revascularisatie?". De resultaten toonden een goede tot uitstekende variatie tussen waarnemers aan. Dit laat zien dat verschillende waarnemers in staat zijn om LSCl-gegevens zonder tegenstrijdige interpretaties te analyseren. De correlatie tussen LSCl-uitkomstparameters en momenteel gebruikte klinische parameters voor succesvolle revascularisatie was laag.

Tot slot zijn in hoofdstuk 7 de belangrijkste bevindingen samengevat ter beantwoording van de vijf onderzoeksvragen van dit proefschrift. Ook zijn de beperkingen van het onderzoek in deze dissertatie besproken, werden aanbevelingen gedaan voor toekomstig onderzoek en werden implicaties beschreven voor de klinische praktijk. LSCI kan duidelijke, onmiddellijke perfusiebeelden maken van de microcirculatie in de voet en het ulcus van mensen met diabetes. LSCI kan op een niet-invasieve manier een volledige voet scannen en de perfusie in en rond een voetulcus meten. Het systeem is betrouwbaar en stabiel, en heeft een lage inter- en intra-beoordelaars variatie. We hebben ook een tracking algoritme ontwikkeld voor de analyse van LSCI 
data dat het gebruik van LSCI in de klinische praktijk verbetert. Bovendien vertoont LSCl zowel overeenkomsten als verschillen met de momenteel gebruikte niet-invasieve bloeddrukmetingen. Dit indiceert dat deze techniek op een nieuwe en andere manier perfusie meet dan de momenteel gebruikte technieken. In ons cohort zijn we er echter niet in geslaagd om perfusie, zoals gemeten met LSCI, te koppelen aan klinische uitkomstparameters zoals genezing van een ulcus of succesvolle revascularisatie. Met andere woorden, de techniek zoals die nu wordt gebruikt heeft nog geen toegevoegde waarde in de zorg. Ondanks dit huidige gebrek aan toepasbaarheid, is deze nieuwe niet-invasieve optische beeldvormingstechniek nog steeds veelbelovend om de klinische praktijk op het gebied van diabetische voetziekte te veranderen. Hiervoor is toekomstig onderzoek nodig om verder te onderzoeken hoe LSCl het beste kan worden gebruikt om de uitkomsten van diabetische voetulcera te verbeteren. 


\section{REFERENTIELIJST}

1. IDF Diabetes Atlas 9th edition 2019 Available online: https://www.diabetesatlas.org/en/ (accessed on Feb 7, 2021).

2. Lazzarini, P.A.; Pacella, R.E.; Armstrong, D.G.; van Netten, J.J. Diabetes-related lowerextremity complications are a leading cause of the global burden of disability. Diabet. Med. 2018.

3. $\quad$ van Netten, J.J.; Bus, S.A.; Apelqvist, J.; Lipsky, B.A.; Hinchliffe, R.J.; Game, F.; Rayman, G.; Lazzarini, P.A.; Forsythe, R.O.; Peters, E.J.; et al. Definitions and criteria for diabetic foot disease on behalf of the International Working Group on the Diabetic Foot. 2020, 19.

4. Alexiadou, K.; Doupis, J. Management of diabetic foot ulcers. Diabetes Ther. 2012, 3, 1-15, doi:10.1007/s13300-012-0004-9.

5. Kerr, M.; Rayman, G.; Jeffcoate, W.J. Cost of diabetic foot disease to the National Health Service in England. Diabet. Med. 2014, 31, 1498-1504.

6. $\quad$ Prompers, L.; Huijberts, M.; Schaper, N.; Apelqvist, J.; Bakker, K.; Edmonds, M.; Holstein, P.; Jude, E.; Jirkovska, A.; Mauricio, D.; et al. Resource utilisation and costs associated with the treatment of diabetic foot ulcers. Prospective data from the Eurodiale Study. Diabetologia 2008, 51, 1826-1834.

7. Prompers, L.; Schaper, N.; Apelqvist, J.; Edmonds, M.; Jude, E.; Mauricio, D.; Uccioli, L.; Urbancic, V.; Bakker, K.; Holstein, P.; et al. Prediction of outcome in individuals with diabetic foot ulcers: Focus on the differences between individuals with and without peripheral arterial disease. The EURODIALE Study. Diabetologia 2008, 51, 747-755.

8. Hinchliffe, R.; Brownrigg, J.; Apelqvist, J.; Boyko, E.J.; Fitridge, R.; Mills, J.; Reekers, J.; Shearman, C.; Zierler, R.; Schaper, N.; et al. IWGDF guidance on the diagnosis, prognosis and management of peripheral artery disease in patients with foot ulcers in diabetes. Diabetes. Metab. Res. Rev. 2016, 32, 37-44.

9. Hinchliffe, R.J.; Forsythe, R.O.; Apelqvist, J.; Boyko, E.J.; Fitridge, R.; Hong, J.P.; Katsanos, K.; Mills, J.L.; Nikol, S.; Reekers, J.; et al. Guidelines on diagnosis, prognosis, and management of peripheral artery disease in patients with foot ulcers and diabetes (IWGDF 2019 update). Diabetes. Metab. Res. Rev. 2020, 36.

10. Forsythe, R.O.; Apelqvist, J.; Boyko, E.J.; Fitridge, R.; Hong, J.P.; Katsanos, K.; Mills, J.L.; Nikol, S.; Reekers, J.; Venermo, M.; et al. Performance of prognostic markers in the prediction of wound healing or amputation among patients with foot ulcers in diabetes: A systematic review. Diabetes. Metab. Res. Rev. 2020, 36, e3278.

11. Lipsky, B.A.; Aragón-Sánchez, J.; Diggle, M.; Embil, J.; Kono, S.; Lavery, L.; Senneville, É.; Urbančič-Rovan, V.; Van Asten, S. IWGDF guidance on the diagnosis and management of foot infections in persons with diabetes. Diabetes. Metab. Res. Rev. 2016, 32, 45-74.

12. Ganapathy, P.; Tamminedi, T.; Qin, Y.; Nanney, L.; Cardwell, N.; Pollins, A.; Sexton, K.; Yadegar, J. Dual-imaging system for burn depth diagnosis. Burns 2014, 40, 67-81.

13. de M. Matheus, A.S.; Clemente, E.L.S.; de Lourdes Guimarães Rodrigues, M.; Torres Valença, D.C.; Gomes, M.B. Assessment of microvascular endothelial function in type 1 diabetes using laser speckle contrast imaging. J. Diabetes Complications 2017, 31, 753-757. 
14. Jayanthy, A.K.; Sujatha, N.; Reddy, M.R.; Narayanamoorthy, V.B. Non invasive blood flow assessment in diabetic foot ulcer using laser speckle contrast imaging technique. In Proceedings of the Arch Ophthalmol; Wax, A., Backman, V., Eds.; International Society for Optics and Photonics, 2014; Vol. 8952, p. 89521D.

15. Mennes, O.A.; van Netten, J.J.; Slart, R.H.J..; Steenbergen, W. Novel Optical Techniques for Imaging Microcirculation in the Diabetic Foot. Curr. Pharm. Des. 2018, 24, 1304-1316. 



\section{ABOUT THE AUTHOR}


Onno August Mennes was born in December 1989 in Utrecht, The Netherlands. He studied Technical Medicine at the University of Twente, also in The Netherlands. He graduated in 2016 in the specialization 'Medical Imaging \& Intervention'. During his graduation project, he started work on the assessment of microcirculation in the foot of people with diabetes with Laser Speckle Contrast Imaging at the Ziekenhuis Groep Twente (ZGT) in Almelo. After his graduation, this project was expanded in a $\mathrm{PhD}$ project which was performed at the Biomedical Photonic Imaging group at the University of Twente under supervision of prof. dr. ir. W. Steenbergen, prof. dr. R.H.J.A. Slart and dr. J.J. van Netten. During his PhD project, Onno also worked at the ZGT as technical physician (2016-2020). Currently, Onno is working as clinical engineer at MED-EL. In his free time he is a home brewer and bee keeper. 

LIST OF PUBLICATIONS 


\section{PAPERS}

Mennes, O.A., Van Netten, J.J., Slart, R.H.J.A., Steenbergen, W. (2018). Novel Optical Techniques for Imaging Microcirculation in the Diabetic Foot.

Current Pharmaceutical Design, 24(1-13).

DOI:10.2174/1381612824666180302141902

Mennes, O.A., Van Netten, J.J., Van Baal, J.G., Steenbergen, W. (2019) Assessment of Microcirculation in the Diabetic Foot with Laser Speckle Contrast Imaging.

Physioloical. Measurement, 40(065002).

DOI:10.1088/1361-6579/ab2058

Mennes, O.A., Van Netten, J.J., Van Baal, J.G., Steenbergen, W. The Association Between Foot and Ulcer Microcirculation Measured with Laser Speckle Contrast Imaging and Healing of Diabetic Foot Ulcers.

Submitted to Journal of Clinical Medicine.

Mennes, O.A. ${ }^{c o}$, Selles, M. ${ }^{c o}$, Van Netten, J.J., Van Baal, J.G., Steenbergen, W., Slart, R.H.J.A. Semi-Automatic Tracking of Laser Speckle Contrast Images of Microcirculation in Diabetic Foot Ulcers.

Diagnostics 2020; 10(12): 1054.

DOI: 10.3390/diagnostics10121054

Wermelink, B. ${ }^{\mathrm{CO}}$, Mennes, O.A. ${ }^{\mathrm{CO}}$, Van Baal, J.G., Steenbergen, W., Geelkerken R.H. Laser Speckle Contrast Imaging During Endovascular Revascularization Procedures in Patients with Peripheral Arterial Occlusive Disease.

Submitted to European Journal of Vascular \& Endovascular Surgery.

\section{PRESENTATIONS}

Oral presentations: 9

Poster presentations: 3 



\section{ACKNOWLEDGEMENTS | DANKWOORD}


Dit dankwoord wil ik gebruiken om de vele mensen te bedanken die direct of indirect bij dit proefschrift betrokken zijn geweest en bijgedragen hebben aan de totstandkoming hiervan. Zonder de hulp en steun van jullie zou dit proefschrift nooit in deze vorm afgerond zijn.

\section{Mijn begeleiders}

Om te beginnen Jaap van Netten. Ik weet nog als de dag van gister dat dit hele avontuur begon toen ik als masterstudent Technische geneeskunde bij jou op hoog niveau (6 ${ }^{\text {de }}$ verdieping ZGT) ging solliciteren op mijn afstudeeropdracht. Vanaf dit eerste moment heb je me dagelijks begeleid in voor- en tegenspoed. Ongeacht de afstand (of het nou aan de andere kant van de wereld, of de andere kant van het land was) kon ik je altijd om raad en advies vragen. Ik kijk uit naar 26 augustus 2026, maar ik verwacht je ook eerder persoonlijk te kunnen danken voor alles wat je me hebt bijgebracht.

Sjef van Baal, als iemand de titel 'leermeester' verdient, dan ben jij dat wel. Vanaf het eerste moment heb je me de passie voor de vaatchirurgie en de complexiteit van de diabetische voet bijgebracht. Je nam me mee in de complexe wereld van de vaatchirurgie en hebt mij laten zien hoe je in lastige periodes toch altijd weer verder kan komen. De steun die, en het vertrouwen dat ik van jou heb gekregen, hebben er toe geleid dat we dit mooie project nog net wat mooier wisten te maken en tot dit eindproduct wisten te krijgen.

Wiendelt Steenbergen. Als hoofd van de BMPI vakgroep was je veel betrokken bij mijn promotieonderzoek en heb ik je vele malen om advies en hulp gevraagd. Ik ben enorm dankbaar voor alle uren dat we de complexe materie konden doorspitten, maar ik ben ook ontzettend dankbaar voor de vele gesprekken die we daarnaast hebben gehad. Het was heel fijn om onder jou te werken omdat je altijd de verhalen zag achter de personen en iedereen stimuleerde om op eigen wijze hun projecten te doorlopen.

Riemer Slart, niet alle wijzen komen uit het Verre Oosten, want sommige komen uit het Hoge Noorden. Vanuit Groningen kwam je vaak op en neer om mij bij te staan met medisch technische vraagstukken en de complexiteit van het uitvoeren van wetenschappelijk onderzoek in de medische wereld. De tijd en geduld waarmee jij mij van feedback en advies hebt voorzien tijdens en na mijn promotietraject heb ik erg gewaardeerd.

\section{De commissie}

Ik heb de grootste dank voor de leden van de commissie die de tijd heeft genomen om mijn proefschrift te lezen, te beoordelen en mij tijdens de verdediging van vragen te voorzien. 


\section{Paranimfen}

Beste Sjoukje en Gerwin. Wat ben ik blij dat jullie hebben toegezegd om mijn paranimfen te zijn tijdens de promotie. Tijdens de lange rit van de promotie hebben jullie altijd achter mij gestaan als het nodig was en 15 minuten lang mijn klaagzang aangehoord als het te veel werd. Dank dat jullie dit nu ruim een uur voor mij wilden volhouden, dat heeft mij door de verdediging heen geholpen.

\section{Co-auteurs}

Beste Mark en Bryan, enorm bedankt voor de fijne samenwerking die we hebben gehad bij het uitvoeren van de twee onderzoekslijnen. Mark, ik vond het mooi te zien hoe jij als student al bij dit onderzoek betrokken bent geraakt, maar het project nooit helemaal los hebt gelaten. Ik vind het meer dan terecht dat we hier samen een hoofdstuk aan hebben kunnen wijden. Bryan, bedankt voor het doorzetten van het LSCl onderzoek in een nieuwe omgeving. Voortgaan op de lopende onderzoekslijnen en samen de stap kunnen zetten naar de HOT laat veel voorspoedige mogelijkheden zien voor de toekomst.

\section{Collega's}

The Space Invaders, mijn kamergenoten waarmee ik alle jaren heb kunnen ploeteren aan al het moois dat de wetenschap te bieden heeft. Met name Sjoukje, mede handbalster op een voetbalveld, waarmee ik veel heb kunnen sparren over METC aanvragen, inclusietrajecten, medisch wetenschap onderzoek, Spotify playlisten met een winstreak van 11 songs en nog vele andere verhalen. Gerwin, altijd optimistisch en altijd vol goede moed. Op het juiste moment pauze houden en ons hoofd resetten met Tik Tak Toe ${ }^{2}$ of andere spellen aan de koffietafel. Niemand kan sprintend deze marathon doorlopen en ik ben blij dat je me dat hebt laten inzien. Ook wil ik Pim bedanken. Wij hebben de wisseling van de wacht gedaan. Waar jij stopte mocht ik verder (maar heb wel vrij snel een afslag genomen). Dank voor alle hulp in de drukte van de afrondfase van je eigen project en fijn dan je mij deze start hebt kunnen geven. Tzu-Lun, dank voor de mooie culinaire uitdagingen die je mij hebt leren waarderen en Hanna, dank voor de vele aquaria die we samen hebben mogen vullen.

Verder alle andere collega's van BMPI. Ik vind het bijzonder om te beseffen hoe vanzelfsprekend de sfeer binnen deze groep was. Altijd positief naar elkaar toe en bijspringen waar mogelijk. Een PhD kan soms een zeer eenzaam traject zijn, maar met jullie lukte het toch om te weten dat we er gezamenlijk voor gingen. Tostivrijdagen blijven een traditie, karaoke is met jullie stiekem toch wel leuk en als het breekt dan printen we het gewoon. Dank voor alle mooie momenten. Applaus!

A special thanks to my colleagues $Y$, Altaf and Mithun. I highly appreciate your support for the Photoacoustic Imaging studies. 
Mijn collega's van het ZGT. Het hele team van de poli en de omloopgang, de secretaresses, de dokters-assistenten, de a(n)iossen en de chirurgen. Enorm bedankt! Daarnaast, mijn speciale dank aan het diabetische voetenteam. Erik, Adriaan, Eline, Joanneke, Marije, Marieke, Patricia, Marieke Marcel en Manon. De harde kern van de omloopgang. Dat jullie tijd en energie vrij maakten om binnen de hectiek van de geneeskunde ook nog wetenschap te verrichten heeft een cruciale rol gespeeld bij dit promotietraject. Gezamenlijk nieuwe projecten opstarten en afronden, het includeren van kandidaten en ondertussen de patiëntenzorg op hoog niveau houden heeft ervoor gezorgd dat dit onderzoek tot zo'n mooi resultaat heeft geleid.

\section{Studenten}

Mijn dank gaat ook uit naar alle studenten die ik tijdens dit traject heb mogen begeleiden en die mij op hun beurt van veel kostbare data hebben voorzien. Naast het verkrijgen van nieuw onderzoeksmateriaal hebben ze mij ook altijd scherp gehouden en mij geleerd om een betere begeleider te worden.

\section{De deelnemers}

Dank aan alle deelnemende personen die belangeloos hun tijd en moeite hebben gestoken in het meewerken bij de onderzoeken met betrekking tot de $\mathrm{LSCl}$ en diabetische voetwonden. Door hun tijd en bereidheid om deel te nemen aan extra onderzoeken en analyses heb ik waardevolle data kunnen verzamelen waarmee nieuwe ontwikkelingen weer een stap dichterbij zijn gekomen.

\section{Vrienden}

MZK. Marije, Joannet, Rob, Daan en Ruud. Niets is planbaar en alles loopt anders dan verwacht. Maar ik ben dankbaar dat ik het met jullie anders heb mogen laten lopen dan we hadden gedacht.

Alle vrienden van ver. Dennis, Martijn, Frank, Bram, Jet, Joris, Lena en Vincent. Door het land verspreid. Ik vind het mooi dat jullie betrokken bleven bij alle updates die vanuit het oosten kwamen en dat jullie mij hielpen om de successen te vieren, maar misschien wel belangrijker, om de tegenslagen door te komen.

Mijn dispuutgenoten, de heren van fatsoen. Jan, Pier, Tjoris en Corneel en alle andere Vrije KonijnenVogels. 10 september was al de mooiste dag van het jaar, maar ik ben blij dat ik op deze bijzondere dag, hem nog net iets mooier mocht maken. Hoog het Hart, Hoog het Lied, Hoog het Bier.

\section{Familie}

Verder mijn speciale dank voor mijn familie die vanuit het hele land en wereld hun steun en support hebben aangeboden. Albert, Joyce, Christel, Freek, Kasper, Julian, Merle, Hester, Jasper, Theo, Anke, Michael, Silke en Vivi. De warme betrokkenheid en vele 
"hulppakketten" die naar mij in Twente zijn gestuurd hebben mij veel goed gedaan. Ein ganz lieber Dank für die wertvolle Unterstützung und das aufrichtige Interesse an meiner Doktorarbeit.

Een speciale dank voor mijn ouders, Albert en Joyce. Bedankt voor de vele jaren steun en vertrouwen die jullie mij geschonken hebben. Van kinds af aan stonden jullie altijd voor mij klaar als dat nodig was en kon ik door jullie de wegen begaan die ik wilde. Ik ben blij en dankbaar dat jullie mijn ouders zijn en ik dit moment samen met jullie mag delen.

Daarnaast mijn beide zussen, Christel en Hester. Bedankt voor alle wijze tips en adviezen die ik van jullie heb gekregen. Of het nu ging om het sparren over de gedoetjes van een $\mathrm{PhD}$ of over de uitdagingen van het ouderschap. Jullie zijn altijd betrokken geweest en wisten met jullie positieve energie en luisterend oor mij altijd weer opgeladen en vol goede moed te krijgen.

Pelle, de traditie eist dat jij (onze stuiterbal op vier poten) ook een plek in dit dankwoord krijgt. Je hebt het verdiend. Het afdwingen van pauzes en het verplichten om naar buiten te gaan en om de zoveel tijd wat zuurstof te inhaleren heeft er oprecht toe geleid dat het traject enerzijds langer duurde dan gepland, maar anderzijds, er voor gezorgd heeft dat ik niet gillend gek werd en vierkante ogen kreeg van mijn beeldscherm.

Daarnaast Nomi en Jidde. Jullie komst heeft mij meer geleerd dan een hele academische carrière ooit zou kunnen doen. Laat mij blijven verbazen, me verwonderen en vooral, laat mij inzien dat er zoveel meer is in het leven.

Nadine, ik zou een dichtbundel kunnen schrijven over alle steun die jij voor mij bent geweest. Ik denk niet dat ik de eindstreep had gehaald als jij niet bij elke meter klaar stond om me aan te moedigen. Bedankt voor het vertrouwen, de steun en het geduld dat je me al deze tijd hebt gegeven. Je betekent meer voor mij dan ik, in een openbaar dankwoord, toelichten kan. 




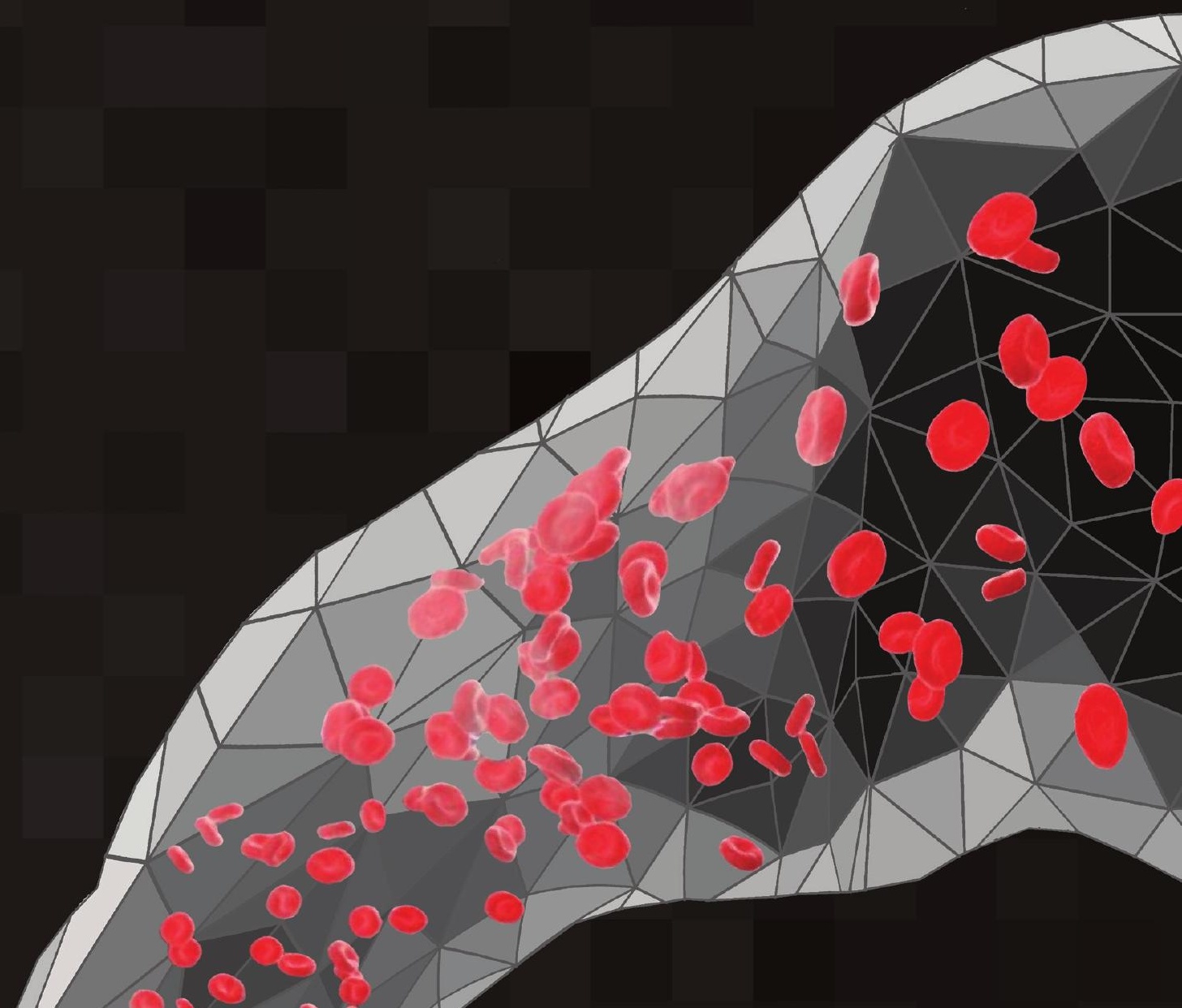

